\title{
General Explicit Solution of Planar Weakly Delayed Linear Discrete Systems and Pasting Its Solutions
}

\author{
Josef Diblík ${ }^{1,2}$ and Hana Halfarová ${ }^{1,2}$ \\ ${ }^{1}$ Department of Mathematics and Descriptive Geometry, Faculty of Civil Engineering, Brno University of Technology, \\ 60200 Brno, Czech Republic \\ ${ }^{2}$ Department of Mathematics, Faculty of Electrical Engineering, Brno University of Technology, 61600 Brno, Czech Republic
}

Correspondence should be addressed to Josef Diblík; diblik@feec.vutbr.cz

Received 3 September 2013; Accepted 21 October 2013; Published 29 April 2014

Academic Editor: Miroslava Růžičková

Copyright (C) 2014 J. Diblík and H. Halfarová. This is an open access article distributed under the Creative Commons Attribution License, which permits unrestricted use, distribution, and reproduction in any medium, provided the original work is properly cited.

Planar linear discrete systems with constant coefficients and delays $x(k+1)=A x(k)+\sum_{l=1}^{n} B^{l} x_{l}\left(k-m_{l}\right)$ are considered where $k \in \mathbb{Z}_{0}^{\infty}:=\{0,1, \ldots, \infty\}, m_{1}, m_{2}, \ldots, m_{n}$ are constant integer delays, $0<m_{1}<m_{2}<\cdots<m_{n}, A, B^{1}, \ldots, B^{n}$ are constant $2 \times 2$ matrices, and $x: \mathbb{Z}_{-m}^{\infty} \rightarrow \mathbb{R}^{2}$. It is assumed that the considered system is weakly delayed. The characteristic equations of such systems are identical with those for the same systems but without delayed terms. In this case, after several steps, the space of solutions with a given starting dimension $2\left(m_{n}+1\right)$ is pasted into a space with a dimension less than the starting one. In a sense, this situation is analogous to one known in the theory of linear differential systems with constant coefficients and special delays when the initially infinite dimensional space of solutions on the initial interval turns (after several steps) into a finite dimensional set of solutions. For every possible case, explicit general solutions are constructed and, finally, results on the dimensionality of the space of solutions are obtained.

\section{Introduction}

1.1. Preliminary Notions and Properties. We use the following notation: for integers $s, q, s \leq q$, we define $\mathbb{Z}_{s}^{q}:=\{s, s+$ $1, \ldots, q\}$, where $s=-\infty$ or $q=\infty$ is admitted, too. Throughout this paper, using notation $\mathbb{Z}_{s}^{q}$, we always assume $s \leq q$. In the paper, we deal with the discrete planar system

$$
x(k+1)=A x(k)+\sum_{l=1}^{n} B^{l} x_{l}\left(k-m_{l}\right),
$$

where $m_{1}, m_{2}, \ldots, m_{n}$ are constant integer delays, $0<m_{1}<$ $m_{2}<\cdots<m_{n}, k \in \mathbb{Z}_{0}^{\infty}, A, B^{1}, \ldots, B^{n}$ are constant $2 \times 2$ matrices, $A=\left(a_{i j}\right), B^{l}=\left(b_{i j}^{l}\right), i, j=1,2, l=1,2, \ldots, n$, and $x: \mathbb{Z}_{-m_{n}}^{\infty} \rightarrow \mathbb{R}^{2}$. Throughout the paper, we assume that

$$
B^{l} \neq \Theta
$$

where $l=1,2, \ldots, n$ and $\Theta$ is $2 \times 2$ zero matrix. Together with (1), we consider an initial (Cauchy) problem

$$
x(k)=\varphi(k),
$$

where $k=-m_{n},-m_{n}+1, \ldots, 0$ with $\varphi: \mathbb{Z}_{-m_{n}}^{0} \rightarrow \mathbb{R}^{2}$. The existence and uniqueness of the solution of the initial problem (1), (3) on $\mathbb{Z}_{-m_{n}}^{\infty}$ are obvious. We recall that the solution $x$ : $\mathbb{Z}_{-m_{n}}^{\infty} \rightarrow \mathbb{R}^{2}$ of (1), (3) is defined as an infinite sequence

$$
\begin{aligned}
& \left\{x\left(-m_{n}\right)=\varphi\left(-m_{n}\right),\right. \\
& x\left(-m_{n}+1\right)=\varphi\left(-m_{n}+1\right), \ldots, \\
& x(0)=\varphi(0), \\
& \quad x(1), x(2), \ldots, x(k), \ldots\}
\end{aligned}
$$

such that, for any $k \in \mathbb{Z}_{0}^{\infty}$, equality (1) holds.

The space of all initial data (3) with $\varphi: \mathbb{Z}_{-m_{n}}^{0} \rightarrow \mathbb{R}^{2}$ is obviously $2\left(m_{n}+1\right)$-dimensional. Below, we describe the 
fact that, among system (1), there are such systems that their space of solutions, being initially $2\left(m_{n}+1\right)$-dimensional, on a reduced interval turns into a space having a dimension less than $2\left(m_{n}+1\right)$. The problem under consideration (pasting property of solutions) is exactly formulated in Section 1.4.

1.2. Weakly Delayed Systems. We consider system (1) and look for a solution having the form $x(k)=\xi \lambda^{k}$, where $k \in \mathbb{Z}_{-m_{n}}^{\infty}$, $\lambda=$ constant with $\lambda \neq 0$, and $\xi=\left(\xi_{1}, \xi_{2}\right)^{T}$ is a nonzero constant vector. The usual procedure leads to a characteristic equation

$$
D:=\operatorname{det}\left(A+\sum_{l=1}^{n} \lambda^{-m_{l}} B^{l}-\lambda I\right)=0,
$$

where $I$ is the unit $2 \times 2$ matrix. Together with (1), we consider a system with the terms containing delays omitted:

$$
x(k+1)=A x(k)
$$

and its characteristic equation

$$
\operatorname{det}(A-\lambda I)=0 \text {. }
$$

Definition 1. System (1) is called a weakly delayed system if characteristic equations (5), (7) corresponding to systems (1) and (6) are equal, that is, if, for every $\lambda \in \mathbb{C} \backslash\{0\}$,

$$
D=\operatorname{det}\left(A+\sum_{l=1}^{n} \lambda^{-m_{l}} B^{l}-\lambda I\right)=\operatorname{det}(A-\lambda I)
$$

We consider a linear transformation

$$
x(k)=\delta y(k)
$$

with a nonsingular $2 \times 2$ matrix $\mathcal{S}$, then the discrete system for $y$ is

$$
y(k+1)=A_{\mathcal{S}} y(k)+\sum_{l=1}^{n} B_{\mathcal{S}}^{l} y\left(k-m_{l}\right),
$$

with $A_{\mathcal{S}}=\mathcal{S}^{-1} A \mathcal{S}, B_{\mathcal{S}}^{l}=\mathcal{S}^{-1} B^{l} \mathcal{S}$, where $l=1,2, \ldots, n$. We show that a system's property of being one weakly delayed is preserved by every nonsingular linear transformation.

Lemma 2. If system (1) is a weakly delayed system, then its arbitrary linear nonsingular transformation (9) again leads to a weakly delayed system (10).

Proof. It is easy to show that

$$
\operatorname{det}\left(A_{\mathcal{S}}+\sum_{l=1}^{n} \lambda^{-m_{l}} B_{\mathcal{S}}^{l}-\lambda I\right)=\operatorname{det}\left(A_{\mathcal{S}}-\lambda I\right)
$$

holds since

$$
\begin{aligned}
& \operatorname{det}\left(A_{\mathcal{S}}+\sum_{l=1}^{n} \lambda^{-m_{l}} B_{\mathcal{S}}^{l}-\lambda I\right) \\
& =\operatorname{det}\left(A_{\mathcal{S}}+\lambda^{-m_{1}} B_{\mathcal{S}}^{1}+\lambda^{-m_{2}} B_{\mathcal{S}}^{2}\right. \\
& \left.+\cdots+\lambda^{-m_{n}} B_{\delta}^{n}-\lambda I\right) \\
& =\operatorname{det}\left(\mathcal{S}^{-1} A \mathcal{S}+\lambda^{-m_{1}} \mathcal{S}^{-1} B^{1} \mathcal{S}\right. \\
& +\lambda^{-m_{2}} \mathcal{S}^{-1} B^{2} \mathcal{S}+\cdots \\
& \left.+\lambda^{-m_{n}} \mathcal{S}^{-1} B^{n} \mathcal{S}-\lambda \mathcal{S}^{-1} I \mathcal{S}\right) \\
& =\operatorname{det}\left[\mathcal { S } ^ { - 1 } \left(A+\lambda^{-m_{1}} B^{1}+\lambda^{-m_{2}} B^{2}\right.\right. \\
& \left.\left.+\cdots+\lambda^{-m_{n}} B^{n}-\lambda I\right) \delta\right] \\
& =\operatorname{det} \mathcal{S}^{-1} \operatorname{det}\left(A+\lambda^{-m_{1}} B^{1}+\lambda^{-m_{2}} B^{2}\right. \\
& \left.+\cdots+\lambda^{-m_{n}} B^{n}-\lambda I\right) \operatorname{det} \mathcal{S} \\
& =\operatorname{det}\left(A+\lambda^{-m_{1}} B^{1}+\lambda^{-m_{2}} B^{2}\right. \\
& \left.+\cdots+\lambda^{-m_{n}} B^{n}-\lambda I\right) \\
& =\operatorname{det}\left(A+\sum_{l=1}^{n} \lambda^{-m_{l}} B^{l}-\lambda I\right) \text {, } \\
& \operatorname{det}\left(A_{\mathcal{S}}-\lambda I\right)=\operatorname{det}\left(\mathcal{S}^{-1} A \mathcal{S}-\lambda \mathcal{S}^{-1} I \mathcal{S}\right) \\
& =\operatorname{det}\left[\mathcal{S}^{-1}(A-\lambda I) \mathcal{\delta}\right] \\
& =\operatorname{det} \mathcal{S}^{-1} \operatorname{det}(A-\lambda I) \operatorname{det} \mathcal{S} \\
& =\operatorname{det}(A-\lambda I) \text {, } \\
& \operatorname{det}\left(A+\sum_{l=1}^{n} \lambda^{-m_{l}} B^{l}-\lambda I\right)=\operatorname{det}(A-\lambda I) ;
\end{aligned}
$$

that is, equality (8) is assumed.

1.3. Necessary and Sufficient Conditions Determining Weakly Delayed Systems. In the next theorem, we give conditions, in terms of determinants, indicating whether a system is weakly delayed.

Theorem 3. System (1) is a weakly delayed system if and only if the following $3 n+n(n-1) / 2$ conditions hold simultaneously:

$$
\begin{aligned}
& b_{11}^{l}+b_{22}^{l}=0 \\
& \left|\begin{array}{ll}
b_{11}^{l} & b_{12}^{l} \\
b_{21}^{l} & b_{22}^{l}
\end{array}\right|=0
\end{aligned}
$$




$$
\begin{aligned}
& \left|\begin{array}{ll}
a_{11} & a_{12} \\
b_{21}^{l} & b_{22}^{l}
\end{array}\right|+\left|\begin{array}{ll}
b_{11}^{l} & b_{12}^{l} \\
a_{21} & a_{22}
\end{array}\right|=0, \\
& \left|\begin{array}{ll}
b_{11}^{l} & b_{12}^{l} \\
b_{21}^{v} & b_{22}^{v}
\end{array}\right|+\left|\begin{array}{ll}
b_{11}^{v} & b_{12}^{v} \\
b_{21}^{l} & b_{22}^{l}
\end{array}\right|=0,
\end{aligned}
$$

where $l, v=1,2, \ldots, n$ and $v>l$.

Proof. We start with computing determinant $D$ defined by (5). We get

$$
D=\left|\begin{array}{ll}
D_{1} & D_{2} \\
D_{3} & D_{4}
\end{array}\right|,
$$

where

$$
\begin{gathered}
D_{1}=a_{11}+b_{11}^{1} \lambda^{-m_{1}}+b_{11}^{2} \lambda^{-m_{2}}+\cdots+b_{11}^{n} \lambda^{-m_{n}}-\lambda, \\
D_{2}=a_{12}+b_{12}^{1} \lambda^{-m_{1}}+b_{12}^{2} \lambda^{-m_{2}}+\cdots+b_{12}^{n} \lambda^{-m_{n}}, \\
D_{3}=a_{21}+b_{21}^{1} \lambda^{-m_{1}}+b_{21}^{2} \lambda^{-m_{2}}+\cdots+b_{21}^{n} \lambda^{-m_{n}}, \\
D_{4}=a_{22}+b_{22}^{1} \lambda^{-m_{1}}+b_{22}^{2} \lambda^{-m_{2}}+\cdots+b_{22}^{n} \lambda^{-m_{n}}-\lambda .
\end{gathered}
$$

Expanding the determinant on the right-hand side along summands of the first column, we get

$$
\begin{aligned}
D= & \left|\begin{array}{ll}
a_{11} & a_{12}+b_{12}^{1} \lambda^{-m_{1}}+b_{12}^{2} \lambda^{-m_{2}}+\cdots+b_{12}^{n} \lambda^{-m_{n}} \\
a_{21} & a_{22}+b_{22}^{1} \lambda^{-m_{1}}+b_{22}^{2} \lambda^{-m_{2}}+\cdots+b_{22}^{n} \lambda^{-m_{n}}-\lambda
\end{array}\right| \\
& +\lambda^{-m_{1}}\left|\begin{array}{cc}
b_{11}^{1} & a_{12}+b_{12}^{1} \lambda^{-m_{1}}+b_{12}^{2} \lambda^{-m_{2}}+\cdots+b_{12}^{n} \lambda^{-m_{n}} \\
b_{21}^{1} & a_{22}+b_{22}^{1} \lambda^{-m_{1}}+b_{22}^{2} \lambda^{-m_{2}}+\cdots+b_{22}^{n} \lambda^{-m_{n}}-\lambda
\end{array}\right| \\
& +\lambda^{-m_{2}}\left|\begin{array}{cc}
b_{11}^{2} & a_{12}+b_{12}^{1} \lambda^{-m_{1}}+b_{12}^{2} \lambda^{-m_{2}}+\cdots+b_{12}^{n} \lambda^{-m_{n}} \\
b_{21}^{2} & a_{22}+b_{22}^{1} \lambda^{-m_{1}}+b_{22}^{2} \lambda^{-m_{2}}+\cdots+b_{22}^{n} \lambda^{-m_{n}}-\lambda
\end{array}\right| \\
& +\cdots \\
& +\lambda^{-m_{n}}\left|\begin{array}{cc}
b_{11}^{n} & a_{12}+b_{12}^{1} \lambda^{-m_{1}}+b_{12}^{2} \lambda^{-m_{2}}+\cdots+b_{12}^{n} \lambda^{-m_{n}} \\
b_{21}^{n} & a_{22}+b_{22}^{1} \lambda^{-m_{1}}+b_{22}^{2} \lambda^{-m_{2}}+\cdots+b_{22}^{n} \lambda^{-m_{n}}-\lambda
\end{array}\right| \\
& +\lambda\left|\begin{array}{cc}
-1 & a_{12}+b_{12}^{1} \lambda^{-m_{1}}+b_{12}^{2} \lambda^{-m_{2}}+\cdots+b_{12}^{n} \lambda^{-m_{n}} \\
0 & a_{22}+b_{22}^{1} \lambda^{-m_{1}}+b_{22}^{2} \lambda^{-m_{2}}+\cdots+b_{22}^{n} \lambda^{-m_{n}}-\lambda
\end{array}\right| .
\end{aligned}
$$

Expanding each of the above determinants along summands of the second column, we have

$$
\begin{aligned}
D= & \left|\begin{array}{ll}
a_{11} & a_{12} \\
a_{21} & a_{22}
\end{array}\right|+\lambda^{-m_{1}}\left|\begin{array}{ll}
a_{11} & b_{12}^{1} \\
a_{21} & b_{22}^{1}
\end{array}\right|+\lambda^{-m_{2}}\left|\begin{array}{ll}
a_{11} & b_{12}^{2} \\
a_{21} & b_{22}^{2}
\end{array}\right| \\
& +\cdots+\lambda^{-m_{n}}\left|\begin{array}{ll}
a_{11} & b_{12}^{n} \\
a_{21} & b_{22}^{n}
\end{array}\right|+\lambda\left|\begin{array}{cc}
a_{11} & 0 \\
a_{21} & -1
\end{array}\right| \\
& +\lambda^{-m_{1}}\left[\left|\begin{array}{ll}
b_{11}^{1} & a_{12} \\
b_{21}^{1} & a_{22}
\end{array}\right|+\lambda^{-m_{1}}\left|\begin{array}{ll}
b_{11}^{1} & b_{12}^{1} \\
b_{21}^{1} & b_{22}^{1}
\end{array}\right|+\lambda^{-m_{2}}\left|\begin{array}{ll}
b_{11}^{1} & b_{12}^{2} \\
b_{21}^{1} & b_{22}^{2}
\end{array}\right|\right. \\
& \left.+\cdots+\lambda^{-m_{n}}\left|\begin{array}{ll}
b_{11}^{1} & b_{12}^{n} \\
b_{21}^{1} & b_{22}^{n}
\end{array}\right|+\lambda\left|\begin{array}{cc}
b_{11}^{1} & 0 \\
b_{21}^{1} & -1
\end{array}\right|\right]
\end{aligned}
$$

$$
\begin{aligned}
& +\lambda^{-m_{2}}\left[\left|\begin{array}{ll}
b_{11}^{2} & a_{12} \\
b_{21}^{2} & a_{22}
\end{array}\right|+\lambda^{-m_{1}}\left|\begin{array}{ll}
b_{11}^{2} & b_{12}^{1} \\
b_{21}^{2} & b_{22}^{1}
\end{array}\right|+\lambda^{-m_{2}}\left|\begin{array}{ll}
b_{11}^{2} & b_{12}^{2} \\
b_{21}^{2} & b_{22}^{2}
\end{array}\right|\right. \\
& \left.+\cdots+\lambda^{-m_{n}}\left|\begin{array}{ll}
b_{11}^{2} & b_{12}^{n} \\
b_{21}^{2} & b_{22}^{n}
\end{array}\right|+\lambda\left|\begin{array}{cc}
b_{11}^{2} & 0 \\
b_{21}^{2} & -1
\end{array}\right|\right] \\
& +\lambda^{-m_{n}}\left[\left|\begin{array}{ll}
b_{11}^{n} & a_{12} \\
b_{21}^{n} & a_{22}
\end{array}\right|+\lambda^{-m_{1}}\left|\begin{array}{ll}
b_{11}^{n} & b_{12}^{1} \\
b_{21}^{n} & b_{22}^{1}
\end{array}\right|+\lambda^{-m_{2}}\left|\begin{array}{ll}
b_{11}^{n} & b_{12}^{2} \\
b_{21}^{n} & b_{22}^{2}
\end{array}\right|\right. \\
& \left.+\cdots+\lambda^{-m_{n}}\left|\begin{array}{ll}
b_{11}^{n} & b_{12}^{n} \\
b_{21}^{n} & b_{22}^{n}
\end{array}\right|+\lambda\left|\begin{array}{cc}
b_{11}^{n} & 0 \\
b_{21}^{n} & -1
\end{array}\right|\right] \\
& +\lambda\left[\begin{array}{cc}
-1 & a_{12} \\
0 & a_{22}
\end{array}\left|+\lambda^{-m_{1}}\right| \begin{array}{cc}
-1 & b_{12}^{1} \\
0 & b_{22}^{1}
\end{array}\left|+\lambda^{-m_{2}}\right| \begin{array}{cc}
-1 & b_{12}^{2} \\
0 & b_{22}^{2}
\end{array} \mid\right. \\
& \left.+\cdots+\lambda^{-m_{n}}\left|\begin{array}{cc}
-1 & b_{12}^{n} \\
0 & b_{22}^{n}
\end{array}\right|+\lambda\left|\begin{array}{cc}
-1 & 0 \\
0 & -1
\end{array}\right|\right] .
\end{aligned}
$$

After simplification, we get

$$
\begin{aligned}
& D=\left|\begin{array}{cc}
a_{11}-\lambda & a_{12} \\
a_{21} & a_{22}-\lambda
\end{array}\right|-\lambda^{-m_{1}+1}\left(b_{11}^{1}+b_{22}^{1}\right) \\
& -\lambda^{-m_{2}+1}\left(b_{11}^{2}+b_{22}^{2}\right)+\cdots-\lambda^{-m_{n}+1}\left(b_{11}^{n}+b_{22}^{n}\right) \\
& +\lambda^{-m_{1}}\left[\left|\begin{array}{ll}
a_{11} & a_{12} \\
b_{21}^{1} & b_{22}^{1}
\end{array}\right|+\left|\begin{array}{ll}
b_{11}^{1} & b_{12}^{1} \\
a_{21} & a_{22}
\end{array}\right|\right] \\
& +\lambda^{-m_{2}}\left[\left|\begin{array}{ll}
a_{11} & a_{12} \\
b_{21}^{2} & b_{22}^{2}
\end{array}\right|+\left|\begin{array}{ll}
b_{11}^{2} & b_{12}^{2} \\
a_{21} & a_{22}
\end{array}\right|\right] \\
& +\cdots+\lambda^{-m_{n}}\left[\left|\begin{array}{ll}
a_{11} & a_{12} \\
b_{21}^{n} & b_{22}^{n}
\end{array}\right|+\left|\begin{array}{ll}
b_{11}^{n} & b_{12}^{n} \\
a_{21} & a_{22}
\end{array}\right|\right] \\
& +\lambda^{-m_{1}-m_{2}}\left[\left|\begin{array}{ll}
b_{11}^{1} & b_{12}^{1} \\
b_{21}^{2} & b_{22}^{2}
\end{array}\right|+\left|\begin{array}{ll}
b_{11}^{2} & b_{12}^{2} \\
b_{21}^{1} & b_{22}^{1}
\end{array}\right|\right] \\
& +\cdots+\lambda^{-m_{1}-m_{n}}\left[\left|\begin{array}{ll}
b_{11}^{1} & b_{12}^{1} \\
b_{21}^{n} & b_{22}^{n}
\end{array}\right|+\left|\begin{array}{ll}
b_{11}^{n} & b_{12}^{n} \\
b_{21}^{1} & b_{22}^{1}
\end{array}\right|\right]
\end{aligned}
$$

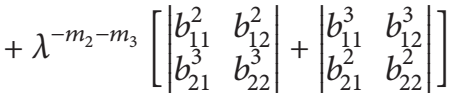

$$
\begin{aligned}
& +\cdots+\lambda^{-m_{2}-m_{n}}\left[\left|\begin{array}{ll}
b_{11}^{2} & b_{12}^{2} \\
b_{21}^{n} & b_{22}^{n}
\end{array}\right|+\left|\begin{array}{ll}
b_{11}^{n} & b_{12}^{n} \\
b_{21}^{2} & b_{22}^{2}
\end{array}\right|\right] \\
& +\cdots+\lambda^{-m_{n-1}-m_{n}}\left[\left|\begin{array}{cc}
b_{11}^{n-1} & b_{12}^{n-1} \\
b_{21}^{n} & b_{22}^{n}
\end{array}\right|+\left|\begin{array}{cc}
b_{11}^{n} & b_{12}^{n} \\
b_{21}^{n-1} & b_{22}^{n-1}
\end{array}\right|\right] \\
& +\lambda^{-2 m_{1}}\left|\begin{array}{ll}
b_{11}^{1} & b_{12}^{1} \\
b_{21}^{1} & b_{22}^{1}
\end{array}\right|+\lambda^{-2 m_{2}}\left|\begin{array}{ll}
b_{11}^{2} & b_{12}^{2} \\
b_{21}^{2} & b_{22}^{2}
\end{array}\right| \\
& +\cdots+\lambda^{-2 m_{n}}\left|\begin{array}{ll}
b_{11}^{n} & b_{12}^{n} \\
b_{21}^{n} & b_{22}^{n}
\end{array}\right| \text {. }
\end{aligned}
$$


Now we see that for (8) to hold; that is,

$$
\begin{aligned}
D & =\operatorname{det}\left(A+\sum_{l=1}^{n} \lambda^{-m_{l}} B^{l}-\lambda I\right) \\
& =\operatorname{det}(A-\lambda I) \\
& =\left|\begin{array}{cc}
a_{11}-\lambda & a_{12} \\
a_{21} & a_{22}-\lambda
\end{array}\right|,
\end{aligned}
$$

conditions (13)-(16) are both necessary and sufficient.

Lemma 4. Conditions (13)-(16) are equivalent to

$$
\begin{gathered}
\operatorname{tr} B^{l}=\operatorname{det} B^{l}=0, \\
\operatorname{det}\left(A+B^{l}\right)=\operatorname{det} A, \\
\operatorname{det}\left(B^{l}+B^{v}\right)=0,
\end{gathered}
$$

where $l, v=1,2, \ldots, n$ and $v>l$.

Proof. (I) We show that assumptions (13)-(16) imply (23)(25). It is obvious that condition (23) is equivalent to (13), (14). Now we consider

$$
\operatorname{det}\left(A+B^{l}\right)=\left|\begin{array}{ll}
a_{11}+b_{11}^{l} & a_{12}+b_{12}^{l} \\
a_{21}+b_{21}^{l} & a_{22}+b_{22}^{l}
\end{array}\right| .
$$

Expanding the determinant on the right-hand side along summands of the first column and then expanding each of the determinants along summands of the second column, we have

$$
\begin{aligned}
\operatorname{det}\left(A+B^{l}\right)= & \left|\begin{array}{ll}
a_{11} & a_{12}+b_{12}^{l} \\
a_{21} & a_{22}+b_{22}^{l}
\end{array}\right|+\left|\begin{array}{ll}
b_{11}^{l} & a_{12}+b_{12}^{l} \\
b_{21}^{l} & a_{22}+b_{22}^{l}
\end{array}\right| \\
= & \left|\begin{array}{ll}
a_{11} & a_{12} \\
a_{21} & a_{22}
\end{array}\right|+\left|\begin{array}{ll}
a_{11} & b_{12}^{l} \\
a_{21} & b_{22}^{l}
\end{array}\right| \\
& +\left|\begin{array}{ll}
b_{11}^{l} & a_{12} \\
b_{21}^{l} & a_{22}
\end{array}\right|+\left|\begin{array}{ll}
b_{11}^{l} & b_{12}^{l} \\
b_{21}^{l} & b_{22}^{l}
\end{array}\right| \\
= & {[\text { due to (15) and (16) }]=\operatorname{det} A . }
\end{aligned}
$$

Now we consider

$$
\operatorname{det}\left(B^{l}+B^{v}\right)=\left|\begin{array}{ll}
b_{11}^{l}+b_{11}^{v} & b_{12}^{l}+b_{12}^{v} \\
b_{21}^{l}+b_{21}^{v} & b_{22}^{l}+b_{22}^{v}
\end{array}\right| .
$$

Expanding the determinant on the right-hand side along summands of the first column and then expanding each of the determinants along summands of the second column, we have

$$
\begin{aligned}
\operatorname{det}\left(B^{l}+B^{v}\right)= & \left|\begin{array}{ll}
b_{11}^{l} & b_{12}^{l}+b_{12}^{v} \\
b_{21}^{l} & b_{22}^{l}+b_{22}^{v}
\end{array}\right|+\left|\begin{array}{ll}
b_{11}^{v} & b_{12}^{l}+b_{12}^{v} \\
b_{21}^{v} & b_{22}^{l}+b_{22}^{v}
\end{array}\right| \\
= & \left|\begin{array}{ll}
b_{11}^{l} & b_{12}^{l} \\
b_{21}^{l} & b_{22}^{l}
\end{array}\right|+\left|\begin{array}{ll}
b_{11}^{l} & b_{12}^{v} \\
b_{21}^{l} & b_{22}^{v}
\end{array}\right| \\
& +\left|\begin{array}{ll}
b_{11}^{v} & b_{12}^{l} \\
b_{21}^{v} & b_{22}^{l}
\end{array}\right|+\left|\begin{array}{ll}
b_{11}^{v} & b_{12}^{v} \\
b_{21}^{v} & b_{22}^{v}
\end{array}\right| \\
= & {[\text { due to (15), (17)] }]=0 . }
\end{aligned}
$$

(II) Now we prove that assumptions (23)-(25) imply (13) and (16). Due to equivalence of (13) and (14) with (23), it remains to be shown that (23)-(25) imply (15) and (16).

If (24) holds, then, from computations in (27), we see that

$$
\left|\begin{array}{ll}
a_{11} & b_{12}^{l} \\
a_{21} & b_{22}^{l}
\end{array}\right|+\left|\begin{array}{ll}
b_{11}^{l} & a_{12} \\
b_{21}^{l} & a_{22}
\end{array}\right|+\left|\begin{array}{ll}
b_{11}^{l} & b_{12}^{l} \\
b_{21}^{l} & b_{22}^{l}
\end{array}\right|=0,
$$

and because of (23) we get (15).

Finally, we show that (23) and (25) imply (16). From (29) (using (23)) we get

$$
\operatorname{det}\left(B^{l}+B^{v}\right)=\left|\begin{array}{ll}
b_{11}^{l} & b_{12}^{v} \\
b_{21}^{l} & b_{22}^{v}
\end{array}\right|+\left|\begin{array}{ll}
b_{11}^{v} & b_{12}^{l} \\
b_{21}^{v} & b_{22}^{l}
\end{array}\right|=0,
$$

that is, (16) holds.

1.4. Problem under Consideration. The aim of this paper is to give explicit formulas for solutions of weakly delayed systems and to show that, after several steps, the dimension of the space of all solutions, being initially equal to the dimension $2\left(m_{n}+1\right)$ of the space of initial data (3) generated by discrete functions $\varphi$, is reduced to a dimension less than the initial one on an interval of the form $\mathbb{Z}_{s}^{\infty}$ with an $s>0$. In other words, we will show that the $2\left(m_{n}+1\right)$-dimensional space of all solutions of (1) is pasted to a less-dimensional space of solutions on $\mathbb{Z}_{s}^{\infty}$. This problem is solved directly by explicitly computing the corresponding solutions of the Cauchy problems with each of the cases arising being considered. The underlying idea for such investigation is simple. If (1) is a weakly delayed system, then the corresponding characteristic equation has only two eigenvalues instead of $2\left(m_{n}+1\right)$ eigenvalues in the case of systems with nonweak delays. This explains why the dimension of the space of solutions becomes less than the initial one. The final results (Theorems 10-13) provide the dimension of the space of solutions. Our results generalize the results in $[1,2]$, where system (1) with $n=1$ and $n=2$ was analyzed.

1.5. Auxiliary Formula. For the reader's convenience, we recall one explicit formula (see, e.g., [3]) for the solutions of linear scalar discrete nondelayed equations used in this paper. We consider initial-value problem for the first order linear discrete nonhomogeneous equation

$$
w(k+1)=a w(k)+g(k), \quad w\left(k_{0}\right)=w_{0}, \quad k \in \mathbb{Z}_{k_{0}}^{\infty},
$$


with $a \in \mathbb{C}$ and $g: \mathbb{Z}_{k_{0}}^{\infty} \rightarrow \mathbb{C}$. Then, it is easy to verify that unique solution of this problem is

$$
w(k)=a^{k-k_{0}} w_{0}+\sum_{r=k_{0}}^{k-1} a^{k-1-r} g(r), \quad k \in \mathbb{Z}_{k_{0}+1}^{\infty} .
$$

Throughout the paper, we adopt the customary notation for the sum: $\sum_{i=\ell+s}^{\ell} \mathscr{F}(i)=0$, where $\ell$ is an integer, $s$ is a positive

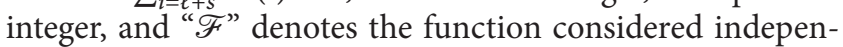
dently of whether it is defined for indicated arguments or not.

Note that the formula (33) is used many times in recent literature to analyze asymptotic properties of solutions of various classes of difference equations, including nonlinear equations. We refer, for example, to [4-8] and to relevant references therein.

\section{General Solution of Weakly Delayed System}

If (8) holds, then (5) and (7) have only two (and the same) roots simultaneously. In order to prove the properties of the family of solutions of (1) formulated in the introduction, we will discuss each combination of roots, that is, the cases of two real and distinct roots, a pair of complex conjugate roots, and, finally, a double real root.

Although computations in Sections 1.2 and 1.3 were performed under assumption that $\lambda \neq 0$, results of this part remain valid also if one or both roots of characteristic equation (7) are zero.

\subsection{Jordan Forms of the Matrix A and Corresponding Solutions} of Problem (1) and (3). It is known that, for every matrix $A$, there exists a nonsingular matrix $S$ transforming it to the corresponding Jordan matrix form $\Lambda$. This means that

$$
\Lambda=S^{-1} A S,
$$

where $\Lambda$ has the following four possible forms (denoted below as $\Lambda_{1}, \Lambda_{2}, \Lambda_{3}, \Lambda_{4}$ ), depending on the roots of the characteristic equation (7), that is, on the roots of

$$
\lambda^{2}-\left(a_{11}+a_{22}\right) \lambda+\left(a_{11} a_{22}-a_{12} a_{21}\right)=0 .
$$

If (35) has two real distinct roots $\lambda_{1}, \lambda_{2}$, then

$$
\Lambda_{1}=\left(\begin{array}{cc}
\lambda_{1} & 0 \\
0 & \lambda_{2}
\end{array}\right)
$$

if the roots are complex conjugate, that is, $\lambda_{1,2}=p \pm i q$ with $q \neq 0$, then

$$
\Lambda_{2}=\left(\begin{array}{cc}
p & q \\
-q & p
\end{array}\right)
$$

and, finally, in the case of one double real root $\lambda_{1,2}=\lambda$, we have either

$$
\Lambda_{3}=\left(\begin{array}{ll}
\lambda & 0 \\
0 & \lambda
\end{array}\right)
$$

or

$$
\Lambda_{4}=\left(\begin{array}{ll}
\lambda & 1 \\
0 & \lambda
\end{array}\right)
$$

The transformation $y(k)=S^{-1} x(k)$ transforms (1) into a system

$$
y(k+1)=\Lambda y(k)+\sum_{l=1}^{n} B^{* l} y\left(k-m_{l}\right), \quad k \in \mathbb{Z}_{0}^{\infty}
$$

with $B^{* l}=S^{-1} B^{l} S, B^{* l}=\left(b_{i j}^{* l}\right), l=1, \ldots, n$, and $i, j=1,2$. Together with (40), we consider an initial problem

$$
y(k)=\varphi^{*}(k)
$$

$k \in \mathbb{Z}_{-m_{n}}^{0}$ with $\varphi^{*}: \mathbb{Z}_{-m_{n}}^{0} \rightarrow \mathbb{R}^{2}$ where $\varphi^{*}(k)=S^{-1} \varphi(k)$ is the initial function corresponding to the initial function $\varphi$ in (3).

Next, we consider all four possible cases (36)-(39) separately.

We define

$$
\begin{array}{r}
\Phi_{1}(k):=\left(0, \varphi_{1}^{*}(k)\right)^{T}, \quad \Phi_{2}(k):=\left(\varphi_{2}^{*}(k), 0\right)^{T}, \\
k \in \mathbb{Z}_{-m_{n}}^{0} .
\end{array}
$$

Assuming that (1) is a weakly delayed system, by Lemma 2, the system (40) is weakly delayed system again.

2.1.1. Case (36) of Two Real Distinct Roots. In this case, we have $\Lambda=\Lambda_{1}$ and $\Lambda_{1}^{k}=\operatorname{diag}\left(\lambda_{1}^{k}, \lambda_{2}^{k}\right)$. The necessary and sufficient conditions (13)-(16) for (40) turn into

$$
\begin{gathered}
b_{11}^{* l}+b_{22}^{* l}=0, \\
\left|\begin{array}{ll}
b_{11}^{* l} & b_{12}^{* l} \\
b_{21}^{* l} & b_{22}^{* l}
\end{array}\right|=b_{11}^{* l} b_{22}^{* l}-b_{12}^{* l} b_{21}^{* l}=0, \\
\left|\begin{array}{cc}
\lambda_{1} & 0 \\
b_{21}^{* l} & b_{22}^{* l}
\end{array}\right|+\left|\begin{array}{cc}
b_{11}^{* l} & b_{12}^{* l} \\
0 & \lambda_{2}
\end{array}\right|=\lambda_{1} b_{22}^{* l}+\lambda_{2} b_{11}^{* l}=0, \\
\left|\begin{array}{ll}
b_{11}^{* l} & b_{12}^{* l} \\
b_{21}^{* v} & b_{22}^{* v}
\end{array}\right|+\left|\begin{array}{ll}
b_{11}^{* v} & b_{12}^{* v} \\
b_{21}^{* l} & b_{22}^{* l}
\end{array}\right|=0 .
\end{gathered}
$$

Since $\lambda_{1} \neq \lambda_{2}$, (43) and (45) yield $b_{11}^{* l}=b_{22}^{* l}=0$, then, from (44), we get $b_{12}^{* l} b_{21}^{* l}=0$, so that either $b_{21}^{* l}=0$ or $b_{12}^{* l}=0$. In view of assumptions $B^{l} \neq \Theta, l=1,2, \ldots, n$, we conclude that only the following cases I, II are possible:

$$
\begin{aligned}
& \text { (I) } b_{11}^{* l}=b_{22}^{* l}=b_{21}^{* l}=0, b_{12}^{* l} \neq 0, l=1,2, \ldots, n, \\
& \text { (II) } b_{11}^{* l}=b_{22}^{* l}=b_{12}^{* l}=0, b_{21}^{* l} \neq 0, l=1,2, \ldots, n .
\end{aligned}
$$

In Theorem 5 both cases I, II are analyzed.

Theorem 5. Let (1) be a weakly delayed system and (35) has two real distinct roots $\lambda_{1}, \lambda_{2}$. If case (I) holds, then the solution 
of the initial problem (1), (3) is $x(k)=S y(k), k \in \mathbb{Z}_{-m_{n}}^{\infty}$, where $y(k)$ has the form

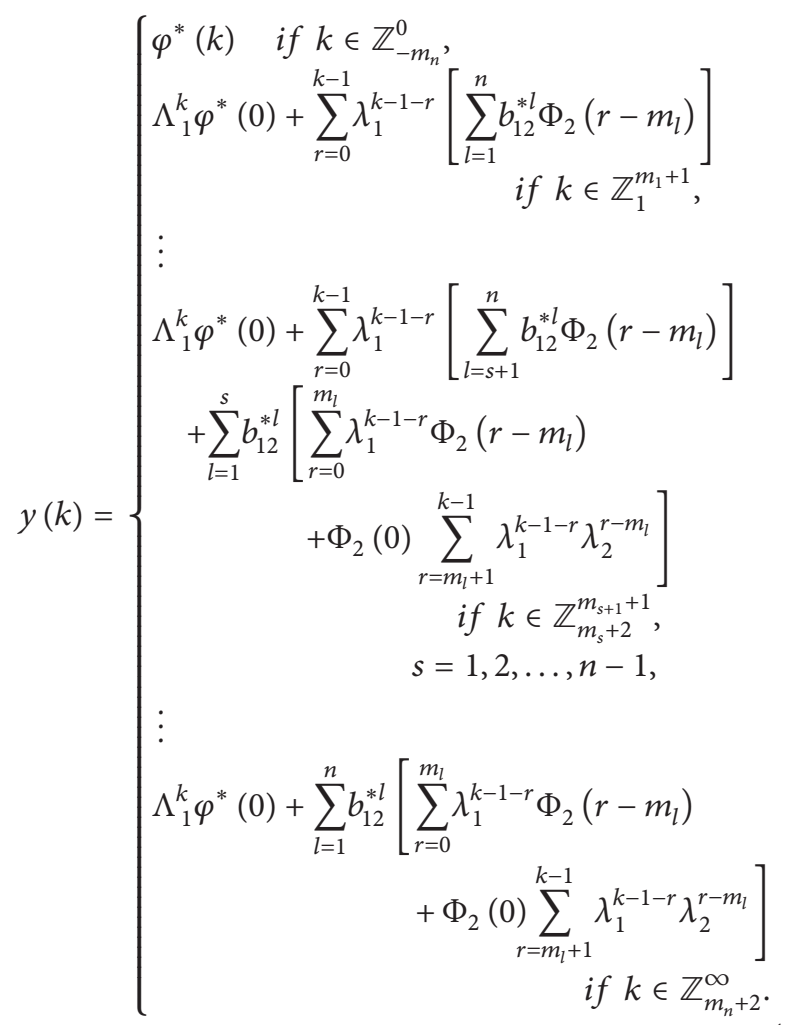

If case (II) is true, then the solution of initial problem (1), (3) is $x(k)=S y(k), k \in \mathbb{Z}_{-m_{n}}^{\infty}$, where $y(k)$ has the form

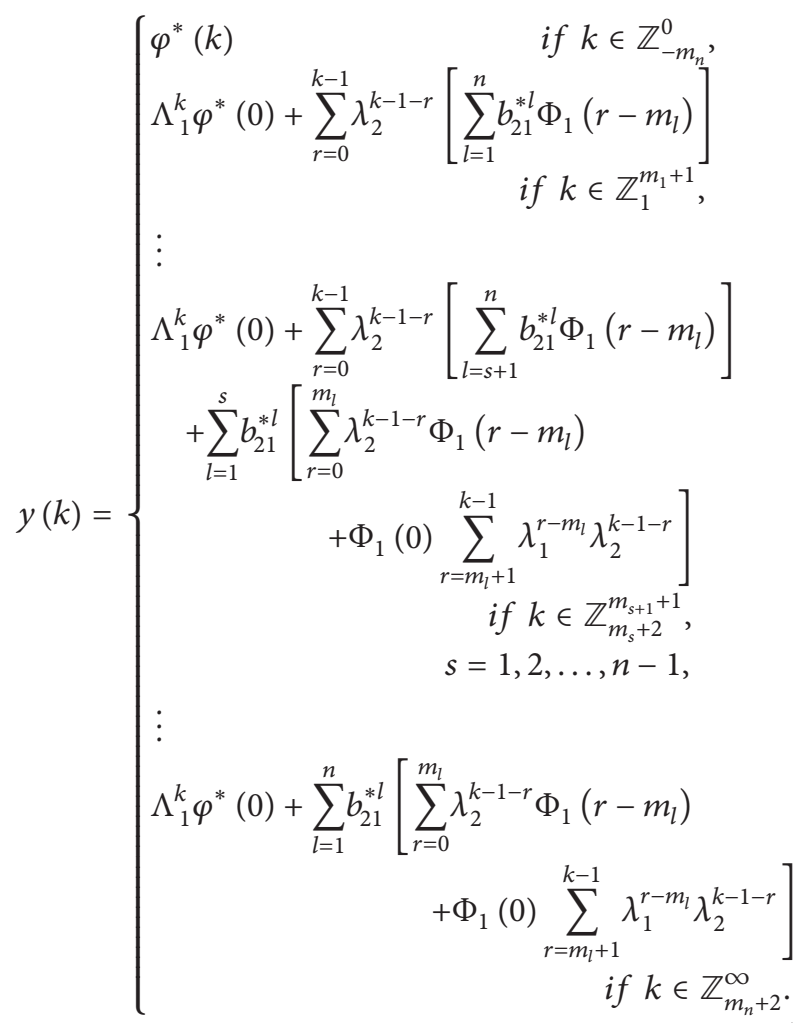

Proof. If case (I) is true, then the transformed system (40) takes the form

$$
\begin{gathered}
y_{1}(k+1)=\lambda_{1} y_{1}(k)+\sum_{l=1}^{n} b_{12}^{* l} y_{2}\left(k-m_{l}\right), \\
y_{2}(k+1)=\lambda_{2} y_{2}(k), \\
k \in \mathbb{Z}_{0}^{\infty},
\end{gathered}
$$

and if case (II) holds, then (40) takes the form

$$
\begin{gathered}
y_{1}(k+1)=\lambda_{1} y_{1}(k), \\
y_{2}(k+1)=\lambda_{2} y_{2}(k)+\sum_{l=1}^{n} b_{21}^{* l} y_{1}\left(k-m_{l}\right),
\end{gathered}
$$$$
k \in \mathbb{Z}_{0}^{\infty} \text {. }
$$

We investigate only the initial problem (49), (50), (41) since the initial problem (51), (52), (41) can be examined in a similar way.

From (50), (41), we get

$$
y_{2}(k)= \begin{cases}\varphi_{2}^{*}(k) & \text { if } k \in \mathbb{Z}_{-m_{n}}^{0}, \\ \lambda_{2}^{k} \varphi_{2}^{*}(0) & \text { if } k \in \mathbb{Z}_{1}^{\infty},\end{cases}
$$

then (49) becomes

$y_{1}(k+1)$

$$
=\left\{\begin{array}{cc}
\lambda_{1} y_{1}(k)+\sum_{l=1}^{n} b_{12}^{* l} \varphi_{2}^{*}\left(k-m_{l}\right) & \text { if } k \in \mathbb{Z}_{0}^{m_{1}}, \\
\lambda_{1} y_{1}(k)+b_{12}^{* 1} \lambda_{2}^{k-m_{1}} \varphi_{2}^{*}(0) & \text { if } k \in \mathbb{Z}_{m_{1}+1}^{m_{2}}, \\
+\sum_{l=2}^{n} b_{12}^{* l} \varphi_{2}^{*}\left(k-m_{l}\right) & \\
\lambda_{1} y_{1}(k)+\sum_{l=1}^{2} b_{12}^{* l} \lambda_{2}^{k-m_{l}} \varphi_{2}^{*}(0) & \\
+\sum_{l=3}^{n} b_{12}^{* l} \varphi_{2}^{*}\left(k-m_{l}\right) & \text { if } k \in \mathbb{Z}_{m_{2}+1}^{m_{3}}, \\
\vdots & \\
\lambda_{1} y_{1}(k)+\sum_{l=1}^{s} b_{12}^{* l} \lambda_{2}^{k-m_{l}} \varphi_{2}^{*}(0) & \\
+\sum_{l=s+1}^{n} b_{12}^{* l} \varphi_{2}^{*}\left(k-m_{l}\right) & \text { if } k \in \mathbb{Z}_{m_{s}+1}^{m_{s+1}}, \\
\vdots & s=3,4, \ldots, n-1, \\
\lambda_{1} y_{1}(k)+\sum_{l=1}^{n} b_{12}^{* l} \lambda_{2}^{k-m_{l}} \varphi_{2}^{*}(0) & \text { if } k \in \mathbb{Z}_{m_{n}+1}^{\infty} .
\end{array}\right.
$$


First, we solve this equation for $k \in \mathbb{Z}_{0}^{m_{1}}$. This means that we consider the problem

$$
\begin{aligned}
y_{1}(k+1)= & \lambda_{1} y_{1}(k) \\
+ & \sum_{l=1}^{n} b_{12}^{* l} \varphi_{2}^{*}\left(k-m_{l}\right), \quad k \in \mathbb{Z}_{0}^{m_{1}}, \\
& y_{1}(0)=\varphi_{1}^{*}(0) .
\end{aligned}
$$

With the aid of formula (33), we get

$$
\begin{array}{r}
y_{1}(k)=\lambda_{1}^{k} \varphi_{1}^{*}(0)+\sum_{r=0}^{k-1} \lambda_{1}^{k-1-r}\left[\sum_{l=1}^{n} b_{12}^{* l} \varphi_{2}^{*}\left(r-m_{l}\right)\right], \\
k \in \mathbb{Z}_{1}^{m_{1}+1} .
\end{array}
$$

Now we solve (54) for $k \in \mathbb{Z}_{m_{1}+1}^{m_{2}}$ with initial data deduced from (56); that is, we consider the problem

$$
\begin{gathered}
y_{1}(k+1)=\lambda_{1} y_{1}(k)+b_{12}^{* 1} \lambda_{2}^{k-m_{1}} \varphi_{2}^{*}(0) \\
+\sum_{l=2}^{n} b_{12}^{* l} \varphi_{2}^{*}\left(k-m_{l}\right), \quad k \in \mathbb{Z}_{m_{1}+1}^{m_{2}}, \\
y_{1}\left(m_{1}+1\right)=\lambda_{1}^{m_{1}+1} \varphi_{1}^{*}(0)+\sum_{r=0}^{m_{1}} \lambda_{1}^{m_{1}-r}\left[\sum_{l=1}^{n} b_{12}^{* l} \varphi_{2}^{*}\left(r-m_{l}\right)\right] .
\end{gathered}
$$

Applying formula (33) we get (for $k \in \mathbb{Z}_{m_{1}+2}^{m_{2}+1}$ )

$$
\begin{aligned}
& y_{1}(k)=\lambda_{1}^{k-\left(m_{1}+1\right)} y_{1}\left(m_{1}+1\right) \\
& +\sum_{r=m_{1}+1}^{k-1} \lambda_{1}^{k-1-r}\left[b_{12}^{* 1} \lambda_{2}^{r-m_{1}} \varphi_{2}^{*}(0)\right. \\
& \left.+\sum_{l=2}^{n} b_{12}^{* l} \varphi_{2}^{*}\left(r-m_{l}\right)\right] \\
& =\lambda_{1}^{k-m_{1}-1}\left[\lambda_{1}^{m_{1}+1} \varphi_{1}^{*}(0)\right. \\
& \left.+\sum_{r=0}^{m_{1}} \lambda_{1}^{m_{1}-r}\left[\sum_{l=1}^{n} b_{12}^{* l} \varphi_{2}^{*}\left(r-m_{l}\right)\right]\right] \\
& +\sum_{r=m_{1}+1}^{k-1} \lambda_{1}^{k-1-r}\left[b_{12}^{* 1} \lambda_{2}^{r-m_{1}} \varphi_{2}^{*}(0)\right. \\
& \left.+\sum_{l=2}^{n} b_{12}^{* l} \varphi_{2}^{*}\left(r-m_{l}\right)\right]
\end{aligned}
$$

$$
\begin{gathered}
=\lambda_{1}^{k} \varphi_{1}^{*}(0)+\sum_{r=0}^{m_{1}} \lambda_{1}^{k-1-r}\left[\sum_{l=1}^{n} b_{12}^{* l} \varphi_{2}^{*}\left(r-m_{l}\right)\right] \\
+\sum_{r=m_{1}+1}^{k-1} \lambda_{1}^{k-1-r}\left[b_{12}^{* 1} \lambda_{2}^{r-m_{1}} \varphi_{2}^{*}(0)\right. \\
\left.+\sum_{l=2}^{n} b_{12}^{* l} \varphi_{2}^{*}\left(r-m_{l}\right)\right] \\
=\lambda_{1}^{k} \varphi_{1}^{*}(0)+\sum_{r=0}^{k-1} \lambda_{1}^{k-1-r}\left[\sum_{l=2}^{n} b_{12}^{* l} \varphi_{2}^{*}\left(r-m_{l}\right)\right] \\
+b_{12}^{* 1}\left[\sum_{r=0}^{m_{1}} \lambda_{1}^{k-1-r} \varphi_{2}^{*}\left(r-m_{1}\right)\right. \\
\left.+\varphi_{2}^{*}(0) \sum_{r=m_{1}+1}^{k-1} \lambda_{1}^{k-1-r} \lambda_{2}^{r-m_{1}}\right] .
\end{gathered}
$$

Now we solve (54) for $k \in \mathbb{Z}_{m_{2}+1}^{m_{3}}$ with initial data deduced from (58); that is, we consider the problem

$$
\begin{aligned}
y_{1}(k+1)= & \lambda_{1} y_{1}(k)+\sum_{l=1}^{2} b_{12}^{* l} \lambda_{2}^{k-m_{l}} \varphi_{2}^{*}(0) \\
& +\sum_{l=3}^{n} b_{12}^{* l} \varphi_{2}^{*}\left(k-m_{l}\right), \quad k \in \mathbb{Z}_{m_{2}+1}^{m_{3}}, \\
y_{1}\left(m_{2}+1\right)= & \lambda_{1}^{m_{2}+1} \varphi_{1}^{*}(0)+\sum_{r=0}^{m_{2}} \lambda_{1}^{m_{2}-r}\left[\sum_{l=2}^{n} b_{12}^{* l} \varphi_{2}^{*}\left(r-m_{l}\right)\right] \\
& +b_{12}^{* 1}\left[\sum_{r=0}^{m_{1}} \lambda_{1}^{m_{2}-r} \varphi_{2}^{*}\left(r-m_{1}\right)\right. \\
& \left.+\varphi_{2}^{*}(0) \sum_{r=m_{1}+1}^{m_{2}} \lambda_{1}^{m_{2}-r} \lambda_{2}^{r-m_{1}}\right]
\end{aligned}
$$

Applying formula (33) yields (for $k \in \mathbb{Z}_{m_{2}+2}^{m_{3}+1}$ )

$$
\begin{aligned}
& y_{1}(k) \\
& =\lambda_{1}^{k-\left(m_{2}+1\right)} y_{1}\left(m_{2}+1\right) \\
& \quad+\sum_{r=m_{2}+1}^{k-1} \lambda_{1}^{k-1-r}\left[\sum_{l=1}^{2} b_{12}^{* l} \lambda_{2}^{r-m_{l}} \varphi_{2}^{*}(0)+\sum_{l=3}^{n} b_{12}^{* l} \varphi_{2}^{*}\left(r-m_{l}\right)\right] \\
& =\lambda_{1}^{k-m_{2}-1}\left[\lambda_{1}^{m_{2}+1} \varphi_{1}^{*}(0)+\sum_{r=0}^{m_{2}} \lambda_{1}^{m_{2}-r}\left[\sum_{l=2}^{n} b_{12}^{* l} \varphi_{2}^{*}\left(r-m_{l}\right)\right]\right.
\end{aligned}
$$




$$
\begin{aligned}
& +b_{12}^{* 1}\left[\sum_{r=0}^{m_{1}} \lambda_{1}^{m_{2}-r} \varphi_{2}^{*}\left(r-m_{1}\right)\right. \\
& \left.\left.+\varphi_{2}^{*}(0) \sum_{r=m_{1}+1}^{m_{2}} \lambda_{1}^{m_{2}-r} \lambda_{2}^{r-m_{1}}\right]\right] \\
& +\sum_{r=m_{2}+1}^{k-1} \lambda_{1}^{k-1-r}\left[\sum_{l=1}^{2} b_{12}^{* l} \lambda_{2}^{r-m_{l}} \varphi_{2}^{*}(0)+\sum_{l=3}^{n} b_{12}^{* l} \varphi_{2}^{*}\left(r-m_{l}\right)\right] \\
& =\lambda_{1}^{k} \varphi_{1}^{*}(0)+\sum_{r=0}^{m_{2}} \lambda_{1}^{k-1-r}\left[\sum_{l=2}^{n} b_{12}^{* l} \varphi_{2}^{*}\left(r-m_{l}\right)\right] \\
& +b_{12}^{* 1}\left[\sum_{r=0}^{m_{1}} \lambda_{1}^{k-1-r} \varphi_{2}^{*}\left(r-m_{1}\right)\right. \\
& \left.+\varphi_{2}^{*}(0) \sum_{r=m_{1}+1}^{m_{2}} \lambda_{1}^{k-1-r} \lambda_{2}^{r-m_{1}}\right] \\
& +\sum_{r=m_{2}+1}^{k-1} \lambda_{1}^{k-1-r}\left[\sum_{l=1}^{2} b_{12}^{* l} \lambda_{2}^{r-m_{l}} \varphi_{2}^{*}(0)\right. \\
& \left.+\sum_{l=3}^{n} b_{12}^{* l} \varphi_{2}^{*}\left(r-m_{l}\right)\right] \\
& =\lambda_{1}^{k} \varphi_{1}^{*}(0)+\sum_{r=0}^{k-1} \lambda_{1}^{k-1-r}\left[\sum_{l=3}^{n} b_{12}^{* l} \varphi_{2}^{*}\left(r-m_{l}\right)\right] \\
& +b_{12}^{* 1}\left[\sum_{r=0}^{m_{1}} \lambda_{1}^{k-1-r} \varphi_{2}^{*}\left(r-m_{1}\right)\right. \\
& \left.+\varphi_{2}^{*}(0) \sum_{r=m_{1}+1}^{k-1} \lambda_{1}^{k-1-r} \lambda_{2}^{r-m_{1}}\right] \\
& +b_{12}^{* 2}\left[\sum_{r=0}^{m_{2}} \lambda_{1}^{k-1-r} \varphi_{2}^{*}\left(r-m_{2}\right)\right. \\
& \left.+\varphi_{2}^{*}(0) \sum_{r=m_{2}+1}^{k-1} \lambda_{1}^{k-1-r} \lambda_{2}^{r-m_{2}}\right] .
\end{aligned}
$$

From (56), (58), and (60) we deduce that expected form of the solution of the initial problem for $k \in \mathbb{Z}_{m_{s-1}+1}^{m_{s}}$ with initial data derived from the solution of previous equation for $k \in \mathbb{Z}_{m_{s-2}+1}^{m_{s-1}}$ is

$$
\begin{aligned}
y_{1}(k)= & \lambda_{1}^{k} \varphi_{1}^{*}(0)+\sum_{r=0}^{k-1} \lambda_{1}^{k-1-r}\left[\sum_{l=s}^{n} b_{12}^{* l} \varphi_{2}^{*}\left(r-m_{l}\right)\right] \\
& +\sum_{l=1}^{s-1} b_{12}^{* l}\left[\sum_{r=0}^{m_{l}} \lambda_{1}^{k-1-r} \varphi_{2}^{*}\left(r-m_{l}\right)\right.
\end{aligned}
$$

$$
\begin{array}{r}
\left.+\varphi_{2}^{*}(0) \sum_{r=m_{l}+1}^{k-1} \lambda_{1}^{k-1-r} \lambda_{2}^{r-m_{l}}\right] \\
\text { if } k \in \mathbb{Z}_{m_{s-1}+2}^{m_{s}+1}
\end{array}
$$

We solve (54) for $k \in \mathbb{Z}_{m_{s}+1}^{m_{s+1}}$ with initial data deduced from (61); that is, we consider the problem

$$
\begin{aligned}
y_{1}(k+1)= & \lambda_{1} y_{1}(k)+\sum_{l=1}^{s} b_{12}^{* l} \lambda_{2}^{k-m_{l}} \varphi_{2}^{*}(0) \\
& +\sum_{l=s+1}^{n} b_{12}^{* l} \varphi_{2}^{*}\left(k-m_{l}\right), \quad k \in \mathbb{Z}_{m_{s}+1}^{m_{s+1}}, \\
y_{1}\left(m_{s}+1\right)= & \lambda_{1}^{m_{s}+1} \varphi_{1}^{*}(0)+\sum_{r=0}^{m_{s}} \lambda_{1}^{m_{s}-r}\left[\sum_{l=s}^{n} b_{12}^{* l} \varphi_{2}^{*}\left(r-m_{l}\right)\right] \\
& +\sum_{l=1}^{s-1} b_{12}^{* l}\left[\sum_{r=0}^{m_{l}} \lambda_{1}^{m_{s}-r} \varphi_{2}^{*}\left(r-m_{l}\right)\right. \\
& \left.+\varphi_{2}^{*}(0) \sum_{r=m_{l}+1}^{m_{s}} \lambda_{1}^{m_{s}-r} \lambda_{2}^{r-m_{l}}\right]
\end{aligned}
$$

Applying formula (33) yields (for $k \in \mathbb{Z}_{m_{s}+2}^{m_{s+1}+1}$ )

$y_{1}(k)$

$$
\begin{aligned}
& =\lambda_{1}^{k-\left(m_{s}+1\right)} y_{1}\left(m_{s}+1\right) \\
& +\sum_{r=m_{s}+1}^{k-1} \lambda_{1}^{k-1-r}\left[\sum_{l=1}^{s} b_{12}^{* l} \lambda_{2}^{r-m_{l}} \varphi_{2}^{*}(0)+\sum_{l=s+1}^{n} b_{12}^{* l} \varphi_{2}^{*}\left(r-m_{l}\right)\right] \\
& =\lambda_{1}^{k-\left(m_{s}+1\right)}\left[\lambda_{1}^{m_{s}+1} \varphi_{1}^{*}(0)+\sum_{r=0}^{m_{s}} \lambda_{1}^{m_{s}-r}\left[\sum_{l=s}^{n} b_{12}^{* l} \varphi_{2}^{*}\left(r-m_{l}\right)\right]\right. \\
& +\sum_{l=1}^{s-1} b_{12}^{* l}\left[\sum_{r=0}^{m_{l}} \lambda_{1}^{m_{s}-r} \varphi_{2}^{*}\left(r-m_{l}\right)\right. \\
& \left.\left.+\varphi_{2}^{*}(0) \sum_{r=m_{l}+1}^{m_{s}} \lambda_{1}^{m_{s}-r} \lambda_{2}^{r-m_{l}}\right]\right] \\
& +\sum_{r=m_{s}+1}^{k-1} \lambda_{1}^{k-1-r}\left[\sum_{l=1}^{s} b_{12}^{* l} \lambda_{2}^{r-m_{l}} \varphi_{2}^{*}(0)\right. \\
& \left.+\sum_{l=s+1}^{n} b_{12}^{* l} \varphi_{2}^{*}\left(r-m_{l}\right)\right] \\
& =\lambda_{1}^{k} \varphi_{1}^{*}(0)+\sum_{r=0}^{m_{s}} \lambda_{1}^{k-1-r}\left[\sum_{l=s}^{n} b_{12}^{* l} \varphi_{2}^{*}\left(r-m_{l}\right)\right]
\end{aligned}
$$




$$
\begin{aligned}
& +\sum_{l=1}^{s-1} b_{12}^{* l}\left[\sum_{r=0}^{m_{l}} \lambda_{1}^{k-1-r} \varphi_{2}^{*}\left(r-m_{l}\right)\right. \\
& =\lambda_{1}^{k} \varphi_{1}^{*}(0)+\sum_{r=0}^{k-1} \lambda_{1}^{k-1-r}\left[\sum_{l=s+1}^{n} b_{12}^{* l} \varphi_{2}^{*}\left(r-m_{l}\right)\right] \\
& \left.+\varphi_{2}^{*}(0) \sum_{r=m_{l}+1}^{m_{s}} \lambda_{1}^{k-1-r} \lambda_{2}^{r-m_{l}}\right] \\
& +\sum_{l=1}^{s} b_{12}^{* l}\left[\sum_{r=0}^{m_{l}} \lambda_{1}^{k-1-r} \varphi_{2}^{*}\left(r-m_{l}\right)\right. \\
& \left.+\varphi_{2}^{*}(0) \sum_{r=m_{l}+1}^{k-1} \lambda_{1}^{k-1-r} \lambda_{2}^{r-m_{l}}\right] . \\
& \left.+\sum_{l=s+1}^{n} b_{12}^{* l} \varphi_{2}^{*}\left(r-m_{l}\right)\right] \\
& =\lambda_{1}^{k} \varphi_{1}^{*}(0)+\sum_{r=0}^{m_{s}} \lambda_{1}^{k-1-r}\left[b_{12}^{* s} \varphi_{2}^{*}\left(r-m_{s}\right)\right. \\
& \left.+\sum_{l=s+1}^{n} b_{12}^{* l} \varphi_{2}^{*}\left(r-m_{l}\right)\right] \\
& +b_{12}^{* 1}\left[\sum_{r=0}^{m_{1}} \lambda_{1}^{k-1-r} \varphi_{2}^{*}\left(r-m_{1}\right)\right. \\
& \left.+\varphi_{2}^{*}(0) \sum_{r=m_{1}+1}^{m_{s}} \lambda_{1}^{k-1-r} \lambda_{2}^{r-m_{1}}\right] \\
& +b_{12}^{* 2}\left[\sum_{r=0}^{m_{2}} \lambda_{1}^{k-1-r} \varphi_{2}^{*}\left(r-m_{2}\right)\right. \\
& \left.+\varphi_{2}^{*}(0) \sum_{r=m_{2}+1}^{m_{s}} \lambda_{1}^{k-1-r} \lambda_{2}^{r-m_{2}}\right] \\
& +\cdots \\
& +b_{12}^{* s-1}\left[\sum_{r=0}^{m_{s-1}} \lambda_{1}^{k-1-r} \varphi_{2}^{*}\left(r-m_{s-1}\right)\right. \\
& \left.+\varphi_{2}^{*}(0) \sum_{r=m_{s-1}+1}^{m_{s}} \lambda_{1}^{k-1-r} \lambda_{2}^{r-m_{s-1}}\right] \\
& +b_{12}^{* 1}\left[\varphi_{2}^{*}(0) \sum_{r=m_{s}+1}^{k-1} \lambda_{1}^{k-1-r} \lambda_{2}^{r-m_{1}}\right] \\
& +b_{12}^{* 2}\left[\varphi_{2}^{*}(0) \sum_{r=m_{s}+1}^{k-1} \lambda_{1}^{k-1-r} \lambda_{2}^{r-m_{2}}\right] \\
& +\ldots \\
& +b_{12}^{* s}\left[\varphi_{2}^{*}(0) \sum_{r=m_{s}+1}^{k-1} \lambda_{1}^{k-1-r} \lambda_{2}^{r-m_{s}}\right] \\
& +\sum_{r=m_{s}+1}^{k-1} \lambda_{1}^{k-1-r}\left[\sum_{l=s+1}^{n} b_{12}^{* l} \varphi_{2}^{*}\left(r-m_{l}\right)\right] \\
& y_{1}(k+1)=\lambda_{1} y_{1}(k)+\sum_{l=1}^{n} b_{12}^{* l} \lambda_{2}^{k-m_{l}} \varphi_{2}^{*}(0), \quad k \in \mathbb{Z}_{m_{n}+1}^{\infty}, \\
& y_{1}\left(m_{n}+1\right)=\lambda_{1}^{m_{n}+1} \varphi_{1}^{*}(0)+\sum_{r=0}^{m_{n}} \lambda_{1}^{m_{n}-r} b_{12}^{* n} \varphi_{2}^{*}\left(r-m_{n}\right) \\
& +\sum_{l=1}^{n-1} b_{12}^{* l}\left[\sum_{r=0}^{m_{l}} \lambda_{1}^{m_{n}-r} \varphi_{2}^{*}\left(r-m_{l}\right)\right. \\
& \left.+\varphi_{2}^{*}(0) \sum_{r=m_{l}+1}^{m_{n}} \lambda_{1}^{m_{n}-r} \lambda_{2}^{r-m_{l}}\right] \\
& y_{1}(k)=\lambda_{1}^{k-\left(m_{n}+1\right)} y_{1}\left(m_{n}+1\right) \\
& +\sum_{r=m_{n}+1}^{k-1} \lambda_{1}^{k-1-r}\left[\sum_{l=1}^{n} b_{12}^{* l} \lambda_{2}^{r-m_{l}} \varphi_{2}^{*}(0)\right] \\
& =\lambda_{1}^{k-\left(m_{n}+1\right)}\left[\lambda_{1}^{m_{n}+1} \varphi_{1}^{*}(0)+\sum_{r=0}^{m_{n}} \lambda_{1}^{m_{n}-r} b_{12}^{* n} \varphi_{2}^{*}\left(r-m_{n}\right)\right. \\
& +\sum_{l=1}^{n-1} b_{12}^{* l}\left[\sum_{r=0}^{m_{l}} \lambda_{1}^{m_{n}-r} \varphi_{2}^{*}\left(r-m_{l}\right)\right. \\
& \left.\left.+\varphi_{2}^{*}(0) \sum_{r=m_{l}+1}^{m_{n}} \lambda_{1}^{m_{n}-r} \lambda_{2}^{r-m_{l}}\right]\right] \\
& +\sum_{r=m_{n}+1}^{k-1} \lambda_{1}^{k-1-r}\left[\sum_{l=1}^{n} b_{12}^{* l} \lambda_{2}^{r-m_{l}} \varphi_{2}^{*}(0)\right] \\
& =\lambda_{1}^{k} \varphi_{1}^{*}(0)+\sum_{r=0}^{m_{n}} \lambda_{1}^{k-1-r} b_{12}^{* n} \varphi_{2}^{*}\left(r-m_{n}\right)
\end{aligned}
$$




$$
\begin{aligned}
& +\sum_{l=1}^{n-1} b_{12}^{* l}\left[\sum_{r=0}^{m_{l}} \lambda_{1}^{k-1-r} \varphi_{2}^{*}\left(r-m_{l}\right)\right. \\
& \left.+\varphi_{2}^{*}(0) \sum_{r=m_{l}+1}^{m_{n}} \lambda_{1}^{k-1-r} \lambda_{2}^{r-m_{l}}\right] \\
& +\sum_{r=m_{n}+1}^{k-1} \lambda_{1}^{k-1-r}\left[\sum_{l=1}^{n} b_{12}^{* l} \lambda_{2}^{r-m_{l}} \varphi_{2}^{*}(0)\right] \\
& =\lambda_{1}^{k} \varphi_{1}^{*}(0)+\sum_{r=0}^{m_{n}} \lambda_{1}^{k-1-r} b_{12}^{* n} \varphi_{2}^{*}\left(r-m_{n}\right) \\
& +b_{12}^{* 1}\left[\sum_{r=0}^{m_{1}} \lambda_{1}^{k-1-r} \varphi_{2}^{*}\left(r-m_{1}\right)\right. \\
& \left.+\varphi_{2}^{*}(0) \sum_{r=m_{1}+1}^{m_{s}} \lambda_{1}^{k-1-r} \lambda_{2}^{r-m_{1}}\right] \\
& +b_{12}^{* 2}\left[\sum_{r=0}^{m_{2}} \lambda_{1}^{k-1-r} \varphi_{2}^{*}\left(r-m_{2}\right)\right. \\
& \left.+\varphi_{2}^{*}(0) \sum_{r=m_{2}+1}^{m_{s}} \lambda_{1}^{k-1-r} \lambda_{2}^{r-m_{2}}\right] \\
& +\cdots \\
& +b_{12}^{* n-1}\left[\sum_{r=0}^{m_{n-1}} \lambda_{1}^{k-1-r} \varphi_{2}^{*}\left(r-m_{n-1}\right)\right. \\
& \left.+\varphi_{2}^{*}(0) \sum_{r=m_{n-1}+1}^{m_{s}} \lambda_{1}^{k-1-r} \lambda_{2}^{r-m_{n-1}}\right] \\
& +b_{12}^{* 1}\left[\varphi_{2}^{*}(0) \sum_{r=m_{s}+1}^{k-1} \lambda_{1}^{k-1-r} \lambda_{2}^{r-m_{1}}\right] \\
& +b_{12}^{* 2}\left[\varphi_{2}^{*}(0) \sum_{r=m_{s}+1}^{k-1} \lambda_{1}^{k-1-r} \lambda_{2}^{r-m_{2}}\right] \\
& +\cdots \\
& +b_{12}^{* n}\left[\varphi_{2}^{*}(0) \sum_{r=m_{n}+1}^{k-1} \lambda_{1}^{k-1-r} \lambda_{2}^{r-m_{n}}\right] \\
& =\lambda_{1}^{k} \varphi_{1}^{*}(0)+\sum_{l=1}^{n} b_{12}^{* l}\left[\sum_{r=0}^{m_{l}} \lambda_{1}^{k-1-r} \varphi_{2}^{*}\left(r-m_{l}\right)\right. \\
& \left.+\varphi_{2}^{*}(0) \sum_{r=m_{l}+1}^{k-1} \lambda_{1}^{k-1-r} \lambda_{2}^{r-m_{l}}\right] .
\end{aligned}
$$

Summing up all particular cases (56)-(65) we have

$$
\begin{aligned}
& \left\{\begin{array}{lr}
\varphi_{1}^{*}(k) & \text { if } k \in \mathbb{Z}_{-m_{n}}^{0}, \\
\lambda_{1}^{k} \varphi_{1}^{*}(0)+\sum_{r=0}^{k-1} \lambda_{1}^{k-1-r}\left[\sum_{l=1}^{n} b_{12}^{* l} \varphi_{2}^{*}\left(r-m_{l}\right)\right]
\end{array}\right. \\
& \text { if } k \in \mathbb{Z}_{1}^{m_{1}+1} \text {, } \\
& \lambda_{1}^{k} \varphi_{1}^{*}(0)+\sum_{r=0}^{k-1} \lambda_{1}^{k-1-r}\left[\sum_{l=2}^{n} b_{12}^{* l} \varphi_{2}^{*}\left(r-m_{l}\right)\right] \\
& +b_{12}^{* 1}\left[\sum_{r=0}^{m_{1}} \lambda_{1}^{k-1-r} \varphi_{2}^{*}\left(r-m_{1}\right)\right. \\
& \left.+\varphi_{2}^{*}(0) \sum_{r=m_{1}+1}^{k-1} \lambda_{1}^{k-1-r} \lambda_{2}^{r-m_{1}}\right] \\
& \text { if } k \in \mathbb{Z}_{m_{1}+2}^{m_{2}+1}, \\
& \lambda_{1}^{k} \varphi_{1}^{*}(0)+\sum_{r=0}^{k-1} \lambda_{1}^{k-1-r}\left[\sum_{l=3}^{n} b_{12}^{* l} \varphi_{2}^{*}\left(r-m_{l}\right)\right] \\
& +b_{12}^{* 1}\left[\sum_{r=0}^{m_{1}} \lambda_{1}^{k-1-r} \varphi_{2}^{*}\left(r-m_{1}\right)\right. \\
& \left.+\varphi_{2}^{*}(0) \sum_{r=m_{1}+1}^{k-1} \lambda_{1}^{k-1-r} \lambda_{2}^{r-m_{1}}\right] \\
& y_{1}(k)=\left\{+b_{12}^{* 2}\left[\sum_{r=0}^{m_{2}} \lambda_{1}^{k-1-r} \varphi_{2}^{*}\left(r-m_{2}\right)\right.\right. \\
& \left.+\varphi_{2}^{*}(0) \sum_{r=m_{2}+1}^{k-1} \lambda_{1}^{k-1-r} \lambda_{2}^{r-m_{2}}\right] \\
& \text { if } k \in \mathbb{Z}_{m_{2}+2}^{m_{3}+1} \text {, } \\
& \lambda_{1}^{k} \varphi_{1}^{*}(0)+\sum_{r=0}^{k-1} \lambda_{1}^{k-1-r}\left[\sum_{l=s+1}^{n} b_{12}^{* l} \varphi_{2}^{*}\left(r-m_{l}\right)\right] \\
& +\sum_{l=1}^{s} b_{12}^{* l}\left[\sum_{r=0}^{m_{l}} \lambda_{1}^{k-1-r} \varphi_{2}^{*}\left(r-m_{l}\right)\right. \\
& \left.+\varphi_{2}^{*}(0) \sum_{r=m_{l}+1}^{k-1} \lambda_{1}^{k-1-r} \lambda_{2}^{r-m_{l}}\right] \\
& \text { if } k \in \mathbb{Z}_{m_{s}+2}^{m_{s+1}+1}, \\
& \lambda_{1}^{k} \varphi_{1}^{*}(0) \\
& +\sum_{l=1}^{n} b_{12}^{* l}\left[\sum_{r=0}^{m_{l}} \lambda_{1}^{k-1-r} \varphi_{2}^{*}\left(r-m_{l}\right)\right. \\
& \left.+\varphi_{2}^{*}(0) \sum_{r=m_{l}+1}^{k-1} \lambda_{1}^{k-1-r} \lambda_{2}^{r-m_{l}}\right] \\
& \text { if } k \in \mathbb{Z}_{m_{n}+2}^{\infty} \text {. }
\end{aligned}
$$

Now, taking into account (42), formula (47) is a consequence of (53) and (66). Formula (48) can be proved in a similar way.

Finally, we note that both formulas (47), (48) remain valid for $b_{12}^{* l}=b_{21}^{* l}=0$. In this case, the transformed system 
(1) reduces to a system without delays. This possibility is excluded by condition (2).

2.1.2. Case (37) of Two Complex Conjugate Roots. The necessary and sufficient conditions (13)-(16) take the forms (43), (44), (46), and

$$
\left|\begin{array}{cc}
p & q \\
b_{21}^{* l} & b_{22}^{* l}
\end{array}\right|+\left|\begin{array}{cc}
b_{11}^{* l} & b_{12}^{* l} \\
-q & p
\end{array}\right|=p\left(b_{11}^{* l}+b_{22}^{* l}\right)+q\left(b_{12}^{* l}-b_{21}^{* l}\right)=0,
$$

where $l, v=1,2, \ldots, n$ and $v>l$.

The system of conditions (43), (44), and (67) gives $b_{12}^{* l}=$ $b_{21}^{* l},\left(b_{11}^{* l}\right)^{2}=-\left(b_{12}^{* l}\right)^{2}$ and admits only one possibility; namely,

$$
b_{11}^{* l}=b_{22}^{* l}=b_{12}^{* l}=b_{21}^{* l}=0 .
$$

Consequently, $B^{* l}=\Theta, B^{l}=\Theta$.

The initial problem (1), (3) reduces to a problem without delay

$$
\begin{gathered}
x(k+1)=A x(k), \\
x(k)=\varphi(k), \quad k \in \mathbb{Z}_{-m_{n}}^{0}
\end{gathered}
$$

and, obviously,

$$
x(k)= \begin{cases}\varphi(k) & \text { if } k \in \mathbb{Z}_{-m_{n}}^{0}, \\ A^{k} \varphi(0) & \text { if } k \in \mathbb{Z}_{1}^{\infty} .\end{cases}
$$

From this discussion, the next theorem follows.

Theorem 6. There exists no weakly delayed system (1) if $\Lambda$ has the form (37).

Finally, we note that the assumption (2) alone excludes this case.

2.1.3. Case (38) of Double Real Root. In this case we have $\Lambda=\Lambda_{3}$ and $\Lambda_{3}^{k}=\operatorname{diag}\left(\lambda^{k}, \lambda^{k}\right)$. For (40), the necessary and sufficient conditions (13)-(16) are reduced to (43), (44), (46), and

$$
\left|\begin{array}{cc}
\lambda & 0 \\
b_{21}^{* l} & b_{22}^{* l}
\end{array}\right|+\left|\begin{array}{cc}
b_{11}^{* l} & b_{12}^{* l} \\
0 & \lambda
\end{array}\right|=\lambda\left(b_{11}^{* l}+b_{22}^{* l}\right)=0,
$$

where $l=1,2, \ldots, n$.

From (43), (44), and (71), we get $b_{12}^{* l} b_{21}^{* l}=-\left(b_{11}^{* l}\right)^{2}$. From the condition (46) we get

$$
b_{11}^{* l} b_{22}^{* v}-b_{12}^{* l} b_{21}^{* v}+b_{22}^{* l} b_{11}^{* v}-b_{21}^{* l} b_{12}^{* v}=0,
$$

where $l, v=1,2, \ldots, n$ and $v>l$. Multiplying (72) by $b_{12}^{* l} b_{12}^{* v}$, we have

$$
\begin{aligned}
& b_{11}^{* l} b_{22}^{* v} b_{12}^{* l} b_{12}^{* v}-\left(b_{12}^{* l}\right)^{2} b_{21}^{* v} b_{12}^{* v} \\
& +b_{22}^{* l} b_{11}^{* v} b_{12}^{* l} b_{12}^{* v}-b_{21}^{* l} b_{12}^{* l}\left(b_{12}^{* v}\right)^{2}=0 .
\end{aligned}
$$

Substituting $b_{12}^{* l} b_{21}^{* l}=-\left(b_{11}^{* l}\right)^{2}, b_{12}^{* v} b_{21}^{* v}=-\left(b_{11}^{* v}\right)^{2}$ into (73) and using (43) we obtain

$$
\begin{aligned}
- & b_{11}^{* l} b_{11}^{* v} b_{12}^{* l} b_{12}^{* v}+\left(b_{12}^{* l}\right)^{2}\left(b_{11}^{* v}\right)^{2} \\
& -b_{11}^{* l} b_{11}^{* v} b_{12}^{* l} b_{12}^{* v}+\left(b_{11}^{* l}\right)^{2}\left(b_{12}^{* v}\right)^{2}=0 .
\end{aligned}
$$

The equation (74) can be written as

$$
\begin{gathered}
\left(b_{12}^{* l} b_{11}^{* v}-b_{12}^{* v} b_{11}^{* l}\right)^{2}=0, \\
b_{12}^{* l} b_{11}^{* v}=b_{12}^{* v} b_{11}^{* l} .
\end{gathered}
$$

Now we will analyse the two possible cases: $b_{12}^{* l} b_{21}^{* l}=0$ and $b_{12}^{* l} b_{21}^{* l} \neq 0$.

For the case $b_{12}^{* l} b_{21}^{* l}=0$, we have from (43), (44) that $b_{11}^{* l}=$ $b_{22}^{* l}=0$ and $b_{12}^{* l}=0$ or $b_{21}^{* l}=0$. For $b_{12}^{* l}=0$ and $b_{21}^{* l} \neq 0$, condition (46) gives $b_{12}^{* v}=0$, where $l, v=1,2, \ldots, n$ and $v>l$. Then, from (43), (44) for $l=v$, we get $b_{11}^{* v}=b_{22}^{* v}=0$ and $b_{21}^{* v} \neq 0$.

For $b_{21}^{* l}=0$ and $b_{12}^{* l} \neq 0$, condition (46) gives $b_{21}^{* v}=0$, where $l, v=1,2, \ldots, n$ and $v>l$, then, from (43), (44) for $l=v$, we get $b_{11}^{* v}=b_{22}^{* v}=0$ and $b_{12}^{* v} \neq 0$.

Now we discuss the case $b_{12}^{* l} b_{21}^{* l} \neq 0$. From conditions (43), (44), we have $b_{12}^{* l} b_{21}^{* l}=-\left(b_{11}^{* l}\right)^{2}$ and $b_{11}^{* l} b_{22}^{* l} \neq 0$. This yields $b_{11}^{* l} \neq 0, b_{22}^{* l} \neq 0$ and, from (75), we have $b_{11}^{* v} \neq 0, b_{12}^{* v} \neq 0$. By conditions (43), (44) for $v=l$, we get $b_{22}^{* v} \neq 0, b_{21}^{* v} \neq 0$.

From the assumptions $B^{l} \neq \Theta$, we conclude that only the following cases ((I), (II), (III)) are possible:

$$
\begin{aligned}
& \text { (I) } b_{11}^{* l}=b_{22}^{* l}=b_{21}^{* l}=0, b_{12}^{* l} \neq 0, \\
& \text { (II) } b_{11}^{* l}=b_{22}^{* l}=b_{12}^{* l}=0, b_{21}^{* l} \neq 0,
\end{aligned}
$$

(III) $b_{12}^{* l} b_{21}^{* l} \neq 0$

where $l=1,2, \ldots, n$.

\subsubsection{Case $b_{12}^{* l} b_{21}^{* l}=0$}

Theorem 7. Let (1) be a weakly delayed system, (35) has a twofold root $\lambda_{1,2}=\lambda, b_{12}^{* l} b_{21}^{* l}=0$ and the matrix $\Lambda$ has the form (38). Then the solution of the initial problem (1), (3) is $x(k)=S y(k), k \in \mathbb{Z}_{-m_{n}}^{\infty}$, where in case $b_{21}^{* l}=0, y(k)$ has the form 


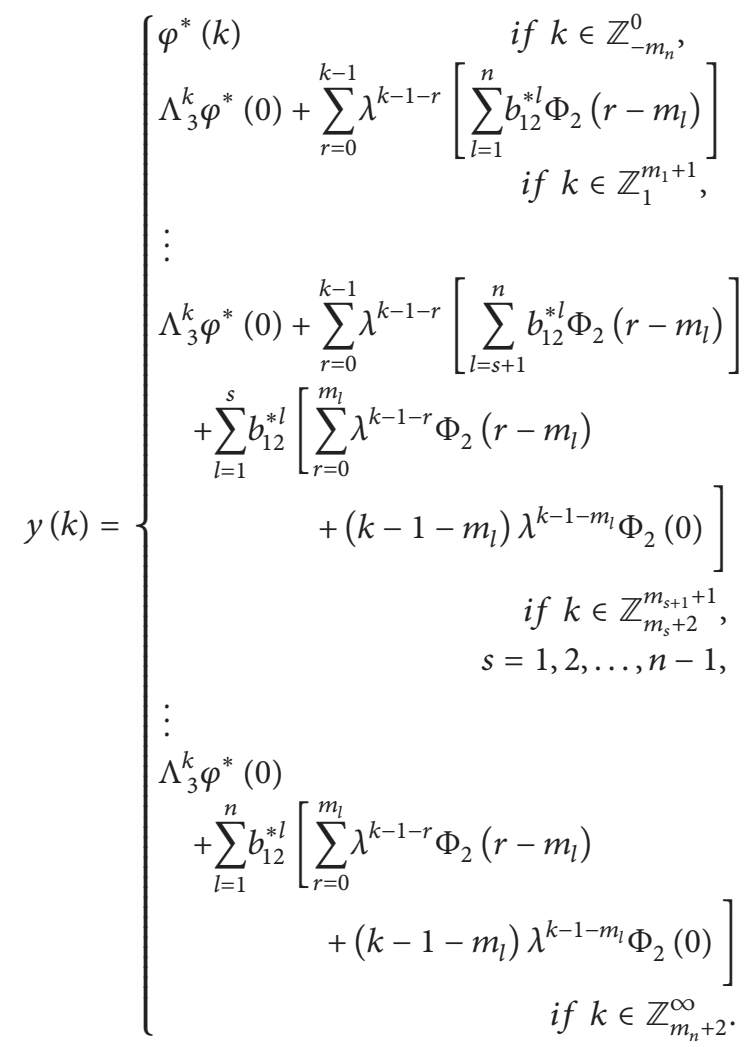

If $b_{12}^{* l}=0$ is true then the solution of initial problem (1), (3) is $x(k)=S y(k), k \in \mathbb{Z}_{-m_{n}}^{\infty}$, where $y(k)$ has the form

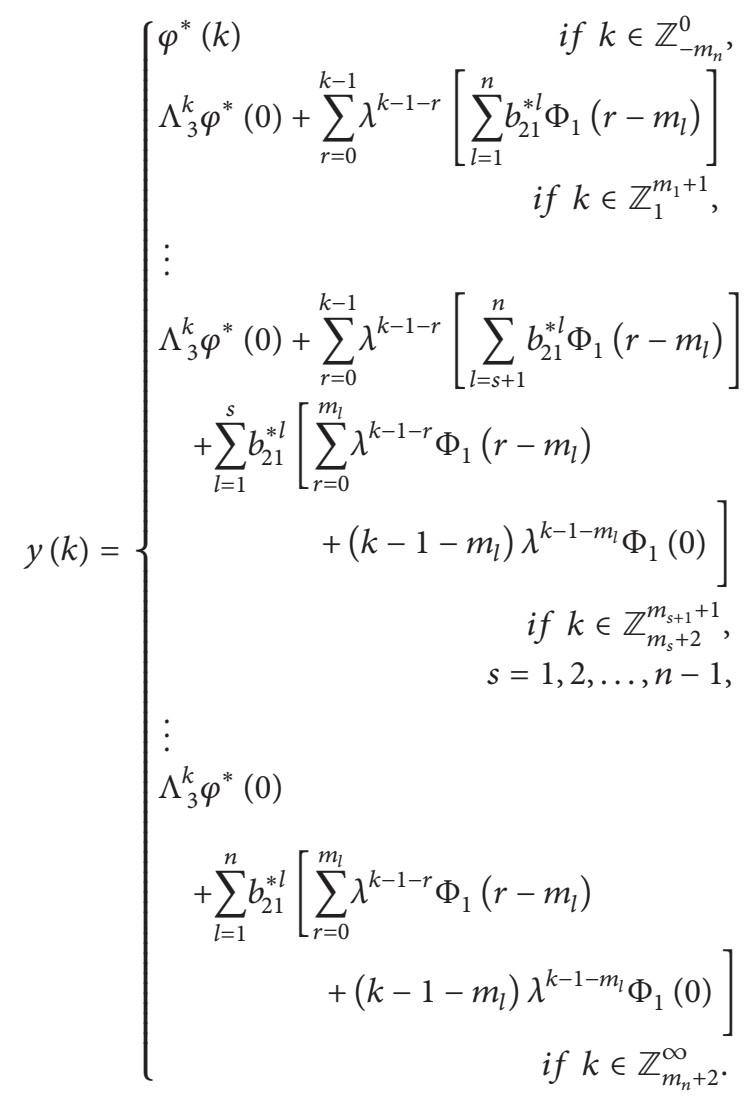

Proof. Case (I) means that $b_{12}^{* l} \neq 0$. Then (40) turns into the system

and, if $b_{21}^{* l} \neq 0,(40)$ turns into the system

$$
\begin{gathered}
y_{1}(k+1)=\lambda y_{1}(k), \\
y_{2}(k+1)=\lambda y_{2}(k)+\sum_{l=1}^{n} b_{21}^{* l} y_{1}\left(k-m_{l}\right), \quad k \in \mathbb{Z}_{0}^{\infty} .
\end{gathered}
$$

System (78) can be solved in much the same way as the systems (49), (50) if we put $\lambda_{1}=\lambda_{2}=\lambda$, and the discussion of the system (79) goes along the same lines as that of the systems (51), (52) with $\lambda_{1}=\lambda_{2}=\lambda$. Formulas (76) and (77) are consequences of (47), (48).

2.1.5. Case $b_{12}^{* l} b_{21}^{* l} \neq 0$. For $k \in \mathbb{Z}_{-m_{n}}^{0}$, we define

$$
\begin{aligned}
\Phi_{l}^{*}(k):=\left(b_{11}^{* l}\left[\varphi_{1}^{*}(k)+\frac{b_{12}^{* 1}}{b_{11}^{* 1}} \varphi_{2}^{*}(k)\right],\right. \\
\left.-\frac{\left(b_{11}^{* l}\right)^{2}}{b_{12}^{* l}}\left[\varphi_{1}^{*}(k)+\frac{b_{12}^{* 1}}{b_{11}^{* 1}} \varphi_{2}^{*}(k)\right]\right)^{T} .
\end{aligned}
$$

Theorem 8. Let system (1) be a weakly delayed system, (35) admits two repeated roots $\lambda_{1,2}=\lambda, b_{12}^{* l} b_{21}^{* l} \neq 0$ and the matrix $\Lambda_{3}$ has the form (38). Then the solution of the initial problem 
(1), (3) is given by $x(k)=S y(k), k \in \mathbb{Z}_{-m_{n}}^{\infty}$, where $y(k)$ has the form

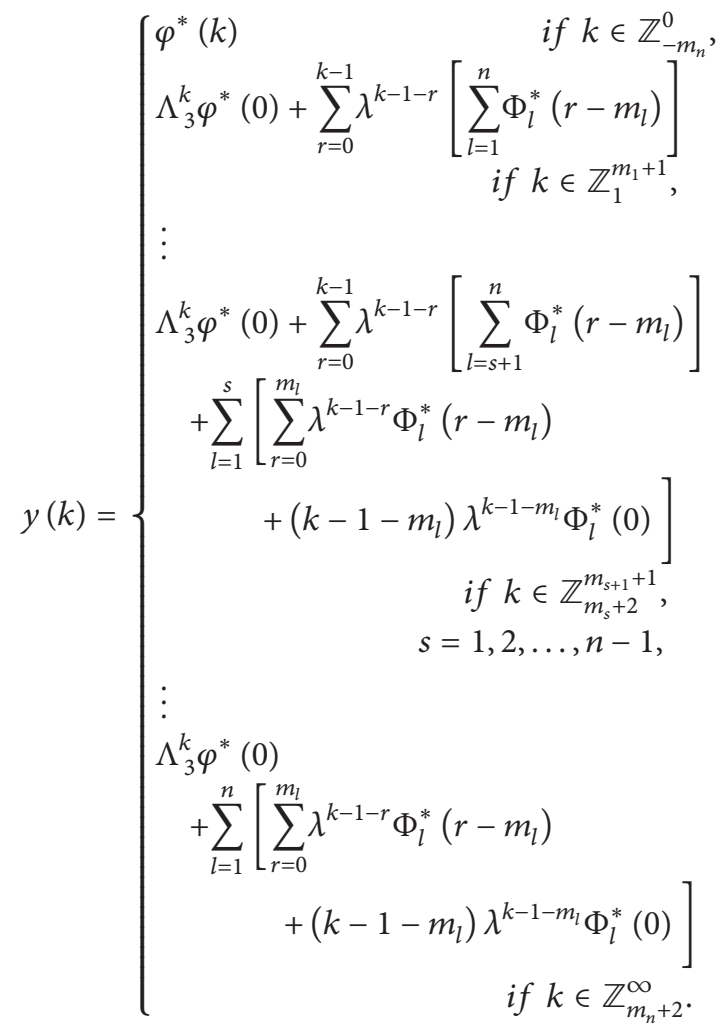

Proof. In this case, all the entries of $B^{* l}$ are nonzero and, from (43), (44), and (71), we get

$$
B^{*}=\left(\begin{array}{cc}
b_{11}^{* l} & b_{12}^{* l} \\
\frac{-\left(b_{11}^{* l}\right)^{2}}{b_{12}^{* l}} & -b_{11}^{* l}
\end{array}\right),
$$

where $l=1,2, \ldots, n$, then, the system (40) reduces to

$$
y_{1}(k+1)=\lambda y_{1}(k)+\sum_{l=1}^{n}\left[b_{11}^{* l} y_{1}\left(k-m_{l}\right)+b_{12}^{* l} y_{2}\left(k-m_{l}\right)\right] \text {, }
$$

$$
\begin{aligned}
& y_{2}(k+1) \\
& \quad=\lambda y_{2}(k)-\sum_{l=1}^{n}\left[\frac{\left(b_{11}^{* l}\right)^{2}}{b_{12}^{* l}} y_{1}\left(k-m_{l}\right)+b_{11}^{* l} y_{2}\left(k-m_{l}\right)\right],
\end{aligned}
$$

where $k \in \mathbb{Z}_{0}^{\infty}$. It is easy to see (multiplying (84) by $b_{12}^{* 1} / b_{11}^{* 1}$ and summing both equations) that

$$
\begin{array}{r}
y_{1}(k+1)+\frac{b_{12}^{* 1}}{b_{11}^{* 1}} y_{2}(k+1)=\lambda\left[y_{1}(k)+\frac{b_{12}^{* 1}}{b_{11}^{* 1}} y_{2}(k)\right], \\
k \in \mathbb{Z}_{0}^{\infty} .
\end{array}
$$

Equation (85) is a homogeneous equation with respect to the unknown expression

$$
y_{1}(k)+\left(\frac{b_{12}^{* 1}}{b_{11}^{* 1}}\right) y_{2}(k)
$$

then, using (33), we obtain

$$
\begin{aligned}
& y_{1}(k)+\frac{b_{12}^{* 1}}{b_{11}^{* 1}} y_{2}(k) \\
& = \begin{cases}\varphi_{1}^{*}(k)+\frac{b_{12}^{* 1}}{b_{11}^{* 1}} \varphi_{2}^{*}(k) & \text { if } k \in \mathbb{Z}_{-m_{n}}^{0}, \\
\lambda^{k}\left[\varphi_{1}^{*}(0)+\frac{b_{12}^{* 1}}{b_{11}^{* 1}} \varphi_{2}^{*}(0)\right] & \text { if } k \in \mathbb{Z}_{1}^{\infty} .\end{cases}
\end{aligned}
$$

With the aid of (87), we rewrite the systems (83), (84) as follows:

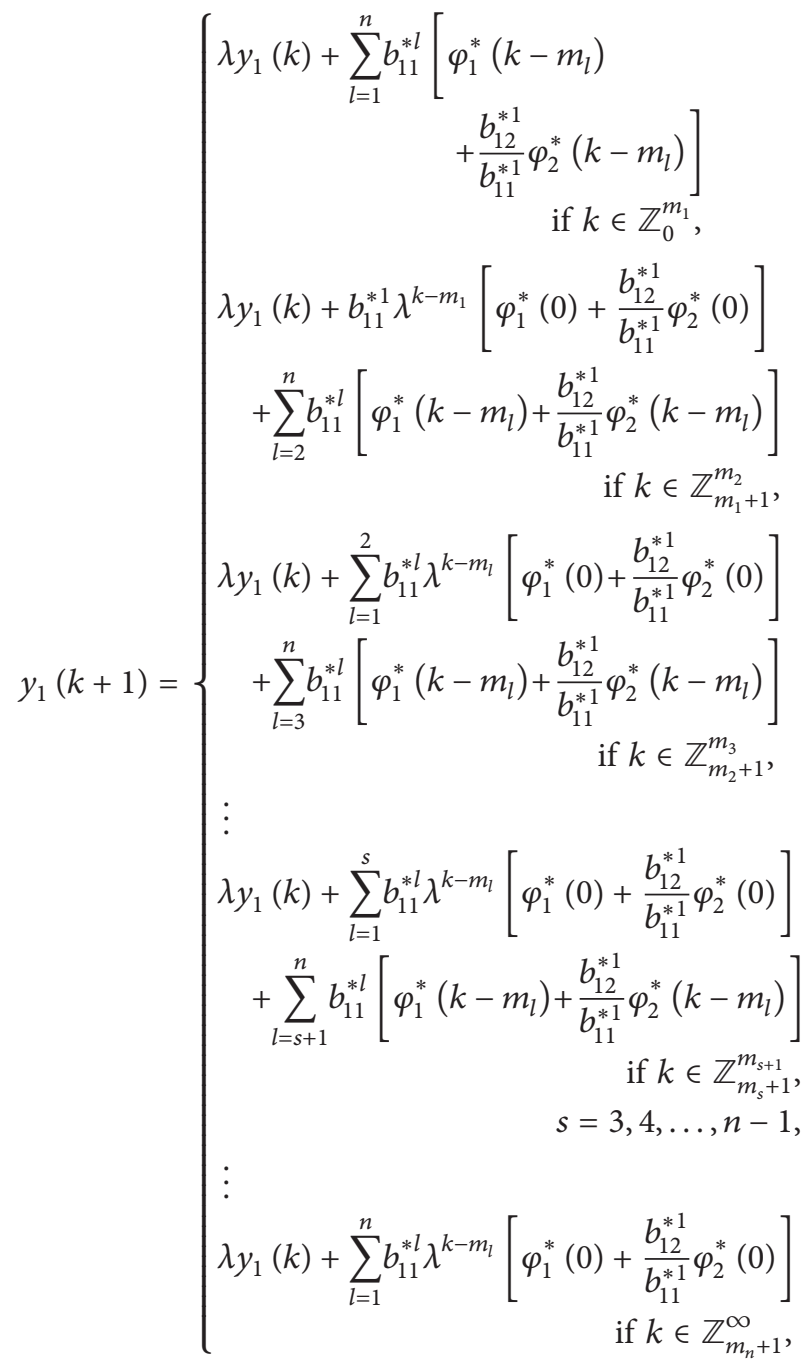




$$
\begin{aligned}
& \int \lambda y_{2}(k)-\sum_{l=1}^{n} \frac{\left(b_{11}^{* l}\right)^{2}}{b_{12}^{* l}}\left[\varphi_{1}^{*}\left(k-m_{l}\right)\right. \\
& \left.+\frac{b_{12}^{* 1}}{b_{11}^{* 1}} \varphi_{2}^{*}\left(k-m_{l}\right)\right] \\
& \lambda y_{2}(k)-\frac{\left(b_{11}^{* 1}\right)^{2}}{b_{12}^{* 1}} \lambda^{k-m_{1}} \\
& \times\left[\varphi_{1}^{*}(0)+\frac{b_{12}^{* 1}}{b_{11}^{* 1}} \varphi_{2}^{*}(0)\right] \\
& -\sum_{l=2}^{n} \frac{\left(b_{11}^{* l}\right)^{2}}{b_{12}^{* l}}\left[\varphi_{1}^{*}\left(k-m_{1}\right)\right. \\
& \left.+\frac{b_{12}^{* 1}}{b_{11}^{* 1}} \varphi_{2}^{*}\left(k-m_{1}\right)\right] \\
& \lambda y_{2}(k)-\sum_{l=1}^{2} \frac{\left(b_{11}^{* l}\right)^{2}}{b_{12}^{* l}}\left[\varphi_{1}^{*}(0)+\frac{b_{12}^{* 1}}{b_{11}^{* 1}} \varphi_{2}^{*}(0)\right] \\
& -\sum_{l=3}^{n} \frac{\left(b_{11}^{* l}\right)^{2}}{b_{12}^{* l}}\left[\varphi_{1}^{*}\left(k-m_{l}\right)\right. \\
& \left.+\frac{b_{12}^{* 1}}{b_{11}^{* 1}} \varphi_{2}^{*}\left(k-m_{l}\right)\right] \\
& \text { if } k \in \mathbb{Z}_{m_{2}+1}^{m_{3}} \text {, } \\
& \lambda y_{2}(k)-\sum_{l=1}^{s} \frac{\left(b_{11}^{* l}\right)^{2}}{b_{12}^{* l}}\left[\varphi_{1}^{*}(0)+\frac{b_{12}^{* 1}}{b_{11}^{* 1}} \varphi_{2}^{*}(0)\right] \\
& -\sum_{l=s+1}^{n} \frac{\left(b_{11}^{* l}\right)^{2}}{b_{12}^{* l}}\left[\varphi_{1}^{*}\left(k-m_{l}\right)\right. \\
& \left.+\frac{b_{12}^{* 1}}{b_{11}^{* 1}} \varphi_{2}^{*}\left(k-m_{l}\right)\right] \\
& \text { if } k \in \mathbb{Z}_{m_{s}+1}^{m_{s+1}} \text {, } \\
& s=3,4, \ldots, n-1 \text {, } \\
& \lambda y_{2}(k)-\sum_{l=1}^{n} \frac{\left(b_{11}^{* l}\right)^{2}}{b_{12}^{* l}} \lambda^{k-m_{l}} \\
& \begin{array}{r}
\times\left[\varphi_{1}^{*}(0)+\frac{b_{12}^{* 1}}{b_{11}^{* 1}} \varphi_{2}^{*}(0)\right] \\
\text { if } k \in \mathbb{Z}_{m_{n}+1}^{\infty} .
\end{array}
\end{aligned}
$$

First, we solve this system for $k \in \mathbb{Z}_{0}^{m_{1}}$ and consider the problems

$$
\begin{gathered}
y_{1}(k+1)=\lambda y_{1}(k) \\
+\sum_{l=1}^{n} b_{11}^{* l}\left[\varphi_{1}^{*}\left(k-m_{l}\right)+\frac{b_{12}^{* 1}}{b_{11}^{* 1}} \varphi_{2}^{*}\left(k-m_{l}\right)\right] \\
\text { if } k \in \mathbb{Z}_{0}^{m_{1}}, \\
y_{1}(0)=\varphi_{1}^{*}(0),
\end{gathered}
$$

$$
\begin{gathered}
y_{2}(k+1)=\lambda y_{2}(k) \\
-\sum_{l=1}^{n} \frac{\left(b_{11}^{* l}\right)^{2}}{b_{12}^{* l}}\left[\varphi_{1}^{*}\left(k-m_{l}\right)+\frac{b_{12}^{* 1}}{b_{11}^{* 1}} \varphi_{2}^{*}\left(k-m_{l}\right)\right] \\
\quad \text { if } k \in \mathbb{Z}_{0}^{m_{1}}, \\
y_{2}(0)=\varphi_{2}^{*}(0) .
\end{gathered}
$$

With the aid of formula (33), we get

$$
\begin{aligned}
& y_{1}(k) \\
& =\lambda^{k} \varphi_{1}^{*}(0)+\sum_{r=0}^{k-1} \lambda^{k-1-r} \\
& \quad \times\left(\sum_{l=1}^{n} b_{11}^{* l}\left[\varphi_{1}^{*}\left(r-m_{l}\right)+\frac{b_{12}^{* 1}}{b_{11}^{* 1}} \varphi_{2}^{*}\left(r-m_{l}\right)\right]\right), \\
& \qquad k \in \mathbb{Z}_{1}^{m_{1}+1}, \\
& y_{2}(k) \quad \lambda^{k} \varphi_{2}^{*}(0)-\sum_{r=0}^{k-1} \lambda^{k-1-r} \\
& \quad \times\left(\sum_{l=1}^{n} \frac{\left(b_{11}^{* l}\right)^{2}}{b_{12}^{* l}}\left[\varphi_{1}^{*}\left(r-m_{l}\right)+\frac{b_{12}^{* 1}}{b_{11}^{* 1}} \varphi_{2}^{*}\left(r-m_{l}\right)\right]\right),
\end{aligned}
$$

Now we solve system (88) for $k \in \mathbb{Z}_{m_{1}+1}^{m_{2}}$; that is, we consider the problem (with initial data deduced from (90), (91))

$$
\begin{aligned}
& y_{1}(k+1) \\
& =\lambda y_{1}(k)+b_{11}^{* 1} \lambda^{k-m_{1}}\left[\varphi_{1}^{*}(0)+\frac{b_{12}^{* 1}}{b_{11}^{* 1}} \varphi_{2}^{*}(0)\right] \\
& \quad+\sum_{l=2}^{n} b_{11}^{* l}\left[\varphi_{1}^{*}\left(k-m_{l}\right)+\frac{b_{12}^{* 1}}{b_{11}^{* 1}} \varphi_{2}^{*}\left(k-m_{l}\right)\right] \\
& \quad \text { if } k \in \mathbb{Z}_{m_{1}+1}^{m_{2}}, \\
& y_{1}\left(m_{1}+1\right) \\
& =\lambda^{m_{1}+1} \varphi_{1}^{*}(0)+\sum_{r=0}^{m_{1}} \lambda^{m_{1}-r} \\
& \quad \times\left(\sum_{l=1}^{n} b_{11}^{* l}\left[\varphi_{1}^{*}\left(r-m_{l}\right)+\frac{b_{12}^{* 1}}{b_{11}^{* 1}} \varphi_{2}^{*}\left(r-m_{l}\right)\right]\right),
\end{aligned}
$$




$$
\begin{aligned}
& y_{2}(k+1) \\
& =\lambda y_{2}(k)-\frac{\left(b_{11}^{* 1}\right)^{2}}{b_{12}^{* 1}} \lambda^{k-m_{1}}\left[\varphi_{1}^{*}(0)+\frac{b_{12}^{* 1}}{b_{11}^{* 1}} \varphi_{2}^{*}(0)\right] \\
& \quad-\sum_{l=2}^{n} \frac{\left(b_{11}^{* l}\right)^{2}}{b_{12}^{* l}}\left[\varphi_{1}^{*}\left(k-m_{1}\right)+\frac{b_{12}^{* 1}}{b_{11}^{* 1}} \varphi_{2}^{*}\left(k-m_{1}\right)\right] \\
& y_{2}\left(m_{1}+1\right) \quad \text { if } k \in \mathbb{Z}_{m_{1}+1}^{m_{2}}, \\
& =\lambda^{m_{1}+1} \varphi_{2}^{*}(0)-\sum_{r=0}^{m_{1}} \lambda^{m_{1}-r} \\
& \quad \times\left(\sum_{l=1}^{n} \frac{\left(b_{11}^{* l}\right)^{2}}{b_{12}^{* l}}\left[\varphi_{1}^{*}\left(r-m_{l}\right)+\frac{b_{12}^{* 1}}{b_{11}^{* 1}} \varphi_{2}^{*}\left(r-m_{l}\right)\right]\right) .
\end{aligned}
$$

Formula (33) yields (for $k \in \mathbb{Z}_{m_{1}+2}^{m_{2}+1}$ )

$y_{1}(k)$

$$
\begin{gathered}
=\lambda^{k-\left(m_{1}+1\right)} y_{1}\left(m_{1}+1\right)+\sum_{r=m_{1}+1}^{k-1} \lambda^{k-1-r} \\
\times\left(b_{11}^{* 1} \lambda^{k-m_{1}}\left[\varphi_{1}^{*}(0)+\frac{b_{12}^{* 1}}{b_{11}^{* 1}} \varphi_{2}^{*}(0)\right]\right. \\
+\sum_{l=2}^{n} b_{11}^{* l}\left[\varphi_{1}^{*}\left(r-m_{l}\right)\right. \\
\left.\left.+\frac{b_{12}^{* 1}}{b_{11}^{* 1}} \varphi_{2}^{*}\left(r-m_{l}\right)\right]\right) \\
=\lambda^{k-m_{1}-1}\left[\lambda^{m_{1}+1} \varphi_{1}^{*}(0)+\sum_{r=0}^{m_{1}} \lambda^{m_{1}-r}\right. \\
\times\left(\sum _ { l = 1 } ^ { n } b _ { 1 1 } ^ { * l } \left[\varphi_{1}^{*}\left(r-m_{l}\right)\right.\right. \\
\left.\left.\left.+\frac{b_{12}^{* 1}}{b_{11}^{* 1}} \varphi_{2}^{*}\left(r-m_{l}\right)\right]\right)\right] \\
+\sum_{r=m_{1}+1}^{k-1} \lambda^{k-1-r}\left(b_{11}^{* 1} \lambda^{k-m_{1}}\left[\varphi_{1}^{*}(0)+\frac{b_{12}^{* 1}}{b_{11}^{* 1}} \varphi_{2}^{*}(0)\right]\right. \\
+\sum_{l=2}^{n} b_{11}^{* l}\left[\varphi_{1}^{*}\left(r-m_{l}\right)\right. \\
\left.\left.+\frac{b_{12}^{* 1}}{b_{11}^{* 1}} \varphi_{2}^{*}\left(r-m_{l}\right)\right]\right)
\end{gathered}
$$

$$
\begin{aligned}
& =\lambda^{k} \varphi_{1}^{*}(0)+\sum_{r=0}^{m_{1}} \lambda^{k-1-r}\left(\sum _ { l = 1 } ^ { n } b _ { 1 1 } ^ { * l } \left[\varphi_{1}^{*}\left(r-m_{l}\right)\right.\right. \\
& \left.\left.+\frac{b_{12}^{* 1}}{b_{11}^{* 1}} \varphi_{2}^{*}\left(r-m_{l}\right)\right]\right) \\
& +\sum_{r=m_{1}+1}^{k-1} \lambda^{k-1-r}\left(b_{11}^{* 1} \lambda^{k-m_{1}}\left[\varphi_{1}^{*}(0)+\frac{b_{12}^{* 1}}{b_{11}^{* 1}} \varphi_{2}^{*}(0)\right]\right. \\
& +\sum_{l=2}^{n} b_{11}^{* l}\left[\varphi_{1}^{*}\left(r-m_{l}\right)\right. \\
& \left.\left.+\frac{b_{12}^{* 1}}{b_{11}^{* 1}} \varphi_{2}^{*}\left(r-m_{l}\right)\right]\right) \\
& =\lambda^{k} \varphi_{1}^{*}(0)+\sum_{r=0}^{k-1} \lambda^{k-1-r}\left(\sum _ { l = 2 } ^ { n } b _ { 1 1 } ^ { * l } \left[\varphi_{1}^{*}\left(r-m_{l}\right)\right.\right.
\end{aligned}
$$$$
\left.\left.+\frac{b_{12}^{* 1}}{b_{11}^{* 1}} \varphi_{2}^{*}\left(r-m_{l}\right)\right]\right)
$$

$$
\begin{gathered}
+\sum_{r=0}^{m_{1}} \lambda^{k-1-r} b_{11}^{* 1}\left[\varphi_{1}^{*}\left(r-m_{1}\right)+\frac{b_{12}^{* 1}}{b_{11}^{* 1}} \varphi_{2}^{*}\left(r-m_{1}\right)\right] \\
+\left(k-1-m_{1}\right) b_{11}^{* 1} \lambda^{k-1-m_{1}}\left[\varphi_{1}^{*}(0)+\frac{b_{12}^{* 1}}{b_{11}^{* 1}} \varphi_{2}^{*}(0)\right] \\
=\lambda^{k} \varphi_{1}^{*}(0)+\sum_{r=0}^{k-1} \lambda^{k-1-r}\left(\sum _ { l = 2 } ^ { n } b _ { 1 1 } ^ { * l } \left[\varphi_{1}^{*}\left(r-m_{l}\right)\right.\right. \\
\left.\left.+\frac{b_{12}^{* 1}}{b_{11}^{* 1}} \varphi_{2}^{*}\left(r-m_{l}\right)\right]\right) \\
+b_{11}^{* 1}\left(\sum_{r=0}^{m_{1}} \lambda^{k-1-r}\left[\varphi_{1}^{*}\left(r-m_{1}\right)+\frac{b_{12}^{* 1}}{b_{11}^{*}} \varphi_{2}^{*}\left(r-m_{1}\right)\right]\right. \\
+\left(k-1-m_{1}\right) \lambda^{k-1-m_{1}}\left[\varphi_{1}^{*}(0)\right. \\
\left.\left.+\frac{b_{12}^{* 1}}{b_{11}^{* 1}} \varphi_{2}^{*}(0)\right]\right)
\end{gathered}
$$

$y_{2}(k)$

$$
\begin{gathered}
=\lambda^{k-\left(m_{1}+1\right)} y_{2}\left(m_{1}+1\right)-\sum_{r=m_{1}+1}^{k-1} \lambda^{k-1-r} \\
\times\left(\frac{\left(b_{11}^{* 1}\right)^{2}}{b_{12}^{* 1}} \lambda^{r-m_{1}}\left[\varphi_{1}^{*}(0)+\frac{b_{12}^{* 1}}{b_{11}^{* 1}} \varphi_{2}^{*}(0)\right]\right. \\
+\sum_{l=2}^{n} \frac{\left(b_{11}^{* l}\right)^{2}}{b_{12}^{* l}}\left[\varphi_{1}^{*}\left(r-m_{l}\right)\right.
\end{gathered}
$$




$$
\begin{aligned}
& \left.\left.+\frac{b_{12}^{* 1}}{b_{11}^{* 1}} \varphi_{2}^{*}\left(r-m_{l}\right)\right]\right) \\
& =\lambda^{k-m_{1}-1}\left[\lambda^{m_{1}+1} \varphi_{2}^{*}(0)-\sum_{r=0}^{m_{1}} \lambda^{m_{1}-r}\right. \\
& \times\left(\sum _ { l = 1 } ^ { n } \frac { ( b _ { 1 1 } ^ { * l } ) ^ { 2 } } { b _ { 1 2 } ^ { * l } } \left[\varphi_{1}^{*}\left(r-m_{l}\right)\right.\right. \\
& \left.\left.\left.+\frac{b_{12}^{* 1}}{b_{11}^{* 1}} \varphi_{2}^{*}\left(r-m_{l}\right)\right]\right)\right] \\
& -\sum_{r=m_{1}+1}^{k-1} \lambda^{k-1-r}\left(\frac{\left(b_{11}^{* 1}\right)^{2}}{b_{12}^{* 1}} \lambda^{r-m_{1}}\left[\varphi_{1}^{*}(0)+\frac{b_{12}^{* 1}}{b_{11}^{* 1}} \varphi_{2}^{*}(0)\right]\right. \\
& +\sum_{l=2}^{n} \frac{\left(b_{11}^{* l}\right)^{2}}{b_{12}^{* l}}\left[\varphi_{1}^{*}\left(r-m_{l}\right)\right. \\
& \left.\left.+\frac{b_{12}^{* 1}}{b_{11}^{* 1}} \varphi_{2}^{*}\left(r-m_{l}\right)\right]\right) \\
& =\lambda^{k} \varphi_{2}^{*}(0)-\sum_{r=0}^{m_{1}} \lambda^{k-1-r}\left(\sum _ { l = 1 } ^ { n } \frac { ( b _ { 1 1 } ^ { * l } ) ^ { 2 } } { b _ { 1 2 } ^ { * l } } \left[\varphi_{1}^{*}\left(r-m_{l}\right)\right.\right. \\
& \left.\left.+\frac{b_{12}^{* 1}}{b_{11}^{* 1}} \varphi_{2}^{*}\left(r-m_{l}\right)\right]\right) \\
& -\sum_{r=m_{1}+1}^{k-1} \lambda^{k-1-r}\left(\frac{\left(b_{11}^{* 1}\right)^{2}}{b_{12}^{* 1}} \lambda^{r-m_{1}}\left[\varphi_{1}^{*}(0)+\frac{b_{12}^{* 1}}{b_{11}^{* 1}} \varphi_{2}^{*}(0)\right]\right. \\
& +\sum_{l=2}^{n} \frac{\left(b_{11}^{* l}\right)^{2}}{b_{12}^{* l}}\left[\varphi_{1}^{*}\left(r-m_{l}\right)\right. \\
& \left.\left.+\frac{b_{12}^{* 1}}{b_{11}^{* 1}} \varphi_{2}^{*}\left(r-m_{l}\right)\right]\right) \\
& =\lambda^{k} \varphi_{2}^{*}(0)-\sum_{r=0}^{k-1} \lambda^{k-1-r}\left(\sum _ { l = 2 } ^ { n } \frac { ( b _ { 1 1 } ^ { * l } ) ^ { 2 } } { b _ { 1 2 } ^ { * l } } \left[\varphi_{1}^{*}\left(r-m_{l}\right)\right.\right. \\
& \left.\left.+\frac{b_{12}^{* 1}}{b_{11}^{* 1}} \varphi_{2}^{*}\left(r-m_{l}\right)\right]\right) \\
& -\sum_{r=0}^{m_{1}} \lambda^{k-1-r} \frac{\left(b_{11}^{* 1}\right)^{2}}{b_{12}^{* 1}}\left[\varphi_{1}^{*}\left(r-m_{1}\right)+\frac{b_{12}^{* 1}}{b_{11}^{* 1}} \varphi_{2}^{*}\left(r-m_{1}\right)\right] \\
& -\left(k-1-m_{1}\right) \lambda^{k-1-m_{1}} \frac{\left(b_{11}^{* 1}\right)^{2}}{b_{12}^{* 1}}\left[\varphi_{1}^{*}(0)+\frac{b_{12}^{* 1}}{b_{11}^{* 1}} \varphi_{2}^{*}(0)\right]
\end{aligned}
$$

Now we solve (88) for $k \in \mathbb{Z}_{m_{2}+1}^{m_{3}}$; that is, we consider the problem (with initial data deduced from (93), (94))

$$
\begin{aligned}
& y_{1}(k+1) \\
& =\lambda y_{1}(k)+\sum_{l=1}^{2} b_{11}^{* l} \lambda^{k-m_{l}}\left[\varphi_{1}^{*}(0)+\frac{b_{12}^{* 1}}{b_{11}^{* 1}} \varphi_{2}^{*}(0)\right] \\
& \quad+\sum_{l=3}^{n} b_{11}^{* l}\left[\varphi_{1}^{*}\left(k-m_{l}\right)+\frac{b_{12}^{* 1}}{b_{11}^{* 1}} \varphi_{2}^{*}\left(k-m_{l}\right)\right] \\
& y_{1}\left(m_{2}+1\right) \quad \text { if } k \in \mathbb{Z}_{m_{2}+1}^{m_{3}}, \\
& =\lambda^{m_{2}+1} \varphi_{1}^{*}(0)+\sum_{r=0}^{m_{2}} \lambda^{m_{2}-r} \\
& \quad\left(\sum_{l=2}^{n} b_{11}^{* l}\left[\varphi_{1}^{*}\left(r-m_{l}\right)+\frac{b_{12}^{* 1}}{b_{11}^{* 1}} \varphi_{2}^{*}\left(r-m_{l}\right)\right]\right) \\
& \quad+b_{11}^{* 1}\left(\sum_{r=0}^{m_{1}} \lambda^{m_{2}-r}\left[\varphi_{1}^{*}\left(r-m_{1}\right)+\frac{b_{12}^{* 1}}{b_{11}^{* 1}} \varphi_{2}^{*}\left(r-m_{1}\right)\right]\right. \\
&
\end{aligned}
$$

$y_{2}(k+1)$

$$
\begin{aligned}
= & \lambda y_{2}(k)-\sum_{l=1}^{2} \frac{\left(b_{11}^{* l}\right)^{2}}{b_{12}^{* l}}\left[\varphi_{1}^{*}(0)+\frac{b_{12}^{* 1}}{b_{11}^{* 1}} \varphi_{2}^{*}(0)\right] \\
& -\sum_{l=3}^{n} \frac{\left(b_{11}^{* l}\right)^{2}}{b_{12}^{* l}}\left[\varphi_{1}^{*}\left(k-m_{l}\right)+\frac{b_{12}^{* 1}}{b_{11}^{* 1}} \varphi_{2}^{*}\left(k-m_{l}\right)\right]
\end{aligned}
$$

if $k \in \mathbb{Z}_{m_{2}+1}^{m_{3}}$, 
Abstract and Applied Analysis

17

$$
\begin{aligned}
& y_{2}\left(m_{2}+1\right) \\
& =\lambda^{m_{2}+1} \varphi_{2}^{*}(0)-\sum_{r=0}^{m_{2}} \lambda^{m_{2}-r} \\
& \quad \times\left(\sum_{l=2}^{n} \frac{\left(b_{11}^{* l}\right)^{2}}{b_{12}^{* l}}\left[\varphi_{1}^{*}\left(r-m_{l}\right)+\frac{b_{12}^{* 1}}{b_{11}^{* 1}} b_{12}^{* l} \varphi_{2}^{*}\left(r-m_{l}\right)\right]\right) \\
& -\frac{\left(b_{11}^{* 1}\right)^{2}}{b_{12}^{* 1}}\left(\sum_{r=0}^{m_{1}} \lambda^{m_{2}-r}\left[\varphi_{1}^{*}\left(r-m_{1}\right)+\frac{b_{12}^{* 1}}{b_{11}^{* 1}} \varphi_{2}^{*}\left(r-m_{1}\right)\right]\right. \\
& \left.\quad+\left(m_{2}-m_{1}\right) \lambda^{m_{2}-m_{1}}\left[\varphi_{1}^{*}(0)+\frac{b_{12}^{* 1}}{b_{11}^{* 1}} \varphi_{2}^{*}(0)\right]\right) .
\end{aligned}
$$

Applying formula (33) yields (for $k \in \mathbb{Z}_{m_{2}+2}^{m_{3}+1}$ )

$y_{1}(k)$

$=\lambda^{k-\left(m_{2}+1\right)} y_{1}\left(m_{2}+1\right)+\sum_{r=m_{2}+1}^{k-1} \lambda^{k-1-r}$

$$
\times\left(\sum_{l=1}^{2} b_{11}^{* l} \lambda^{r-m_{l}}\left[\varphi_{1}^{*}(0)+\frac{b_{12}^{* 1}}{b_{11}^{* 1}} \varphi_{2}^{*}(0)\right]\right.
$$

$\left.+\sum_{l=3}^{n} b_{11}^{* l}\left[\varphi_{1}^{*}\left(r-m_{l}\right)+\frac{b_{12}^{* 1}}{b_{11}^{* 1}} \varphi_{2}^{*}\left(r-m_{l}\right)\right]\right)$

$=\lambda^{k-m_{2}-1}\left[\lambda^{m_{2}+1} \varphi_{1}^{*}(0)+\sum_{r=0}^{m_{2}} \lambda^{m_{2}-r}\right.$

$\times\left(\sum_{l=2}^{n} b_{11}^{* l}\left[\varphi_{1}^{*}\left(r-m_{l}\right)\right.\right.$

$\left.\left.+\frac{b_{12}^{* 1}}{b_{11}^{* 1}} \varphi_{2}^{*}\left(r-m_{l}\right)\right]\right)$

$+b_{11}^{* 1}\left(\sum_{r=0}^{m_{1}} \lambda^{m_{2}-r}\left[\varphi_{1}^{*}\left(r-m_{1}\right)\right.\right.$

$\left.+\frac{b_{12}^{* 1}}{b_{11}^{* 1}} \varphi_{2}^{*}\left(r-m_{1}\right)\right]$

$+\left(m_{2}-m_{1}\right) \lambda^{m_{2}-m_{1}}$

$\left.\left.\times\left[\varphi_{1}^{*}(0)+\frac{b_{12}^{* 1}}{b_{11}^{* 1}} \varphi_{2}^{*}(0)\right]\right)\right]$

$+\sum_{r=m_{2}+1}^{k-1} \lambda^{k-1-r}\left(\sum_{l=1}^{2} b_{11}^{* l} \lambda^{r-m_{l}}\left[\varphi_{1}^{*}(0)+\frac{b_{12}^{* 1}}{b_{11}^{* 1}} \varphi_{2}^{*}(0)\right]\right.$ $+\sum_{l=3}^{n} b_{11}^{* l}\left[\varphi_{1}^{*}\left(r-m_{l}\right)\right.$

$\left.\left.+\frac{b_{12}^{* 1}}{b_{11}^{* 1}} \varphi_{2}^{*}\left(r-m_{l}\right)\right]\right)$

$=\lambda^{k} \varphi_{1}^{*}(0)+\sum_{r=0}^{m_{2}} \lambda^{k-1-r}\left(\sum_{l=2}^{n} b_{11}^{* l}\left[\varphi_{1}^{*}\left(r-m_{l}\right)\right.\right.$

$$
\left.\left.+\frac{b_{12}^{* 1}}{b_{11}^{* 1}} \varphi_{2}^{*}\left(r-m_{l}\right)\right]\right)
$$

$+b_{11}^{* 1}\left(\sum_{r=0}^{m_{1}} \lambda^{k-1-r}\left[\varphi_{1}^{*}\left(r-m_{1}\right)+\frac{b_{12}^{* 1}}{b_{11}^{* 1}} \varphi_{2}^{*}\left(r-m_{1}\right)\right]\right.$

$\left.+\left(m_{2}-m_{1}\right) \lambda^{k-1-m_{1}}\left[\varphi_{1}^{*}(0)+\frac{b_{12}^{* 1}}{b_{11}^{* 1}} \varphi_{2}^{*}(0)\right]\right)$

$+\sum_{r=m_{2}+1}^{k-1} \lambda^{k-1-r}\left(\sum_{l=1}^{2} b_{11}^{* l} \lambda^{r-m_{l}}\left[\varphi_{1}^{*}(0)+\frac{b_{12}^{* 1}}{b_{11}^{* 1}} \varphi_{2}^{*}(0)\right]\right.$

$+\sum_{l=3}^{n} b_{11}^{* l}\left[\varphi_{1}^{*}\left(r-m_{l}\right)\right.$

$\left.\left.+\frac{b_{12}^{* 1}}{b_{11}^{* 1}} \varphi_{2}^{*}\left(r-m_{l}\right)\right]\right)$

$=\lambda^{k} \varphi_{1}^{*}(0)+\sum_{r=0}^{k-1} \lambda^{k-1-r}\left(\sum_{l=3}^{n} b_{11}^{* l}\left[\varphi_{1}^{*}\left(r-m_{l}\right)\right.\right.$

$\left.\left.+\frac{b_{12}^{* 1}}{b_{11}^{* 1}} \varphi_{2}^{*}\left(r-m_{l}\right)\right]\right)$

$+\sum_{r=0}^{m_{2}} \lambda^{k-1-r} b_{11}^{* 2}\left[\varphi_{1}^{*}\left(r-m_{2}\right)+\frac{b_{12}^{* 1}}{b_{11}^{* 1}} \varphi_{2}^{*}\left(r-m_{2}\right)\right]$

$+b_{11}^{* 1}\left(\sum_{r=0}^{m_{1}} \lambda^{k-1-r}\left[\varphi_{1}^{*}\left(r-m_{1}\right)+\frac{b_{12}^{* 1}}{b_{11}^{* 1}} \varphi_{2}^{*}\left(r-m_{1}\right)\right]\right.$

$\left.+\left(m_{2}-m_{1}\right) \lambda^{k-1-m_{1}}\left[\varphi_{1}^{*}(0)+\frac{b_{12}^{* 1}}{b_{11}^{* 1}} \varphi_{2}^{*}(0)\right]\right)$

$+\left(k-1-m_{2}\right)\left(b_{11}^{* 1} \lambda^{k-1-m_{1}}\left[\varphi_{1}^{*}(0)+\frac{b_{12}^{* 1}}{b_{11}^{* 1}} \varphi_{2}^{*}(0)\right]\right.$

$\left.+b_{11}^{* 2} \lambda^{k-1-m_{2}}\left[\varphi_{1}^{*}(0)+\frac{b_{12}^{* 1}}{b_{11}^{* 1}} \varphi_{2}^{*}(0)\right]\right)$

$=\lambda^{k} \varphi_{1}^{*}(0)+\sum_{r=0}^{k-1} \lambda^{k-1-r}\left(\sum_{l=3}^{n} b_{11}^{* l}\left[\varphi_{1}^{*}\left(r-m_{l}\right)\right.\right.$

$\left.\left.+\frac{b_{12}^{* 1}}{b_{11}^{* 1}} \varphi_{2}^{*}\left(r-m_{l}\right)\right]\right)$ 
18

Abstract and Applied Analysis

$$
\begin{aligned}
& +b_{11}^{* 1}\left(\sum_{r=0}^{m_{1}} \lambda^{k-1-r}\left[\varphi_{1}^{*}\left(r-m_{1}\right)+\frac{b_{12}^{* 1}}{b_{11}^{* 1}} \varphi_{2}^{*}\left(r-m_{1}\right)\right]\right. \\
& =\lambda^{k} \varphi_{2}^{*}(0)-\sum_{r=0}^{m_{2}} \lambda^{k-1-r}\left(\sum _ { l = 2 } ^ { n } \frac { ( b _ { 1 1 } ^ { * l } ) ^ { 2 } } { b _ { 1 2 } ^ { * l } } \left[\varphi_{1}^{*}\left(r-m_{l}\right)\right.\right. \\
& \left.+\left(k-1-m_{1}\right) \lambda^{k-1-m_{1}}\left[\varphi_{1}^{*}(0)+\frac{b_{12}^{* 1}}{b_{11}^{* 1}} \varphi_{2}^{*}(0)\right]\right) \\
& \left.\left.+\frac{b_{12}^{* 1}}{b_{11}^{* 1}} \varphi_{2}^{*}\left(r-m_{l}\right)\right]\right) \\
& +b_{11}^{* 2}\left(\sum_{r=0}^{m_{2}} \lambda^{k-1-r}\left[\varphi_{1}^{*}\left(r-m_{2}\right)+\frac{b_{12}^{* 1}}{b_{11}^{* 1}} \varphi_{2}^{*}\left(r-m_{2}\right)\right]\right. \\
& \left.+\left(k-1-m_{2}\right) \lambda^{k-1-m_{2}}\left[\varphi_{1}^{*}(0)+\frac{b_{12}^{* 1}}{b_{11}^{* 1}} \varphi_{2}^{*}(0)\right]\right), \\
& -\frac{\left(b_{11}^{* 1}\right)^{2}}{b_{12}^{* 1}}\left(\sum_{r=0}^{m_{1}} \lambda^{k-1-r}\left[\varphi_{1}^{*}\left(r-m_{1}\right)+\frac{b_{12}^{* 1}}{b_{11}^{* 1}} \varphi_{2}^{*}\left(r-m_{1}\right)\right]\right. \\
& y_{2}(k) \\
& \begin{array}{r}
\left.+\left(m_{2}-m_{1}\right) \lambda^{k-1-m_{1}}\left[\varphi_{1}^{*}(0)+\frac{b_{12}^{* 1}}{b_{11}^{* 1}} \varphi_{2}^{*}(0)\right]\right) \\
-\sum_{r=m_{2}+1}^{k-1} \lambda^{k-1-r}\left(\sum_{l=1}^{2} \frac{\left(b_{11}^{* l}\right)^{2}}{b_{12}^{* l}} \lambda^{r-m_{l}}\left[\varphi_{1}^{*}(0)+\frac{b_{12}^{* 1}}{b_{11}^{* 1}} \varphi_{2}^{*}(0)\right]\right. \\
+\sum_{l=3}^{n} \frac{\left(b_{11}^{* l}\right)^{2}}{b_{12}^{* l}}\left[\varphi_{1}^{*}\left(r-m_{l}\right)\right. \\
\left.\left.+\frac{b_{12}^{* 1}}{b_{11}^{* 1}} \varphi_{2}^{*}\left(r-m_{l}\right)\right]\right) \\
=\lambda^{k} \varphi_{2}^{*}(0)-\sum_{r=0}^{k-1} \lambda^{k-1-r}\left(\sum _ { l = 3 } ^ { n } \frac { ( b _ { 1 1 } ^ { * l } ) ^ { 2 } } { b _ { 1 2 } ^ { * l } } \left[\varphi_{1}^{*}\left(r-m_{l}\right)\right.\right. \\
\left.\left.+\frac{b_{12}^{* 1}}{b_{11}^{* 1}} \varphi_{2}^{*}\left(r-m_{l}\right)\right]\right) \\
+\sum_{r=0}^{m_{2}} \lambda^{k-1-r} \frac{\left(b_{11}^{* 2}\right)^{2}}{b_{12}^{* 2}}\left[\varphi_{1}^{*}\left(r-m_{2}\right)+\frac{b_{12}^{* 1}}{b_{11}^{* 1}} \varphi_{2}^{*}\left(r-m_{2}\right)\right] \\
-\frac{\left(b_{11}^{* 1}\right)^{2}}{b_{12}^{* 1}}\left(\sum _ { r = 0 } ^ { m _ { 1 } } \lambda ^ { k - 1 - r } \left[\varphi_{1}^{*}\left(r-m_{1}\right)\right.\right. \\
+\frac{b_{12}^{* 1}}{\left.b_{11}^{* 1} \varphi_{2}^{*}\left(r-m_{1}\right)\right]} \\
\left.\left.m_{1}\right) \lambda^{k-1-m_{1}}\left[\varphi_{1}^{*}(0)+\frac{b_{12}^{* 1}}{b_{11}^{* 1}} \varphi_{2}^{*}(0)\right]\right)
\end{array} \\
& =\lambda^{k-\left(m_{2}+1\right)} y_{2}\left(m_{2}+1\right)-\sum_{r=m_{2}+1}^{k-1} \lambda^{k-1-r} \\
& \begin{array}{r}
\left.+\left(m_{2}-m_{1}\right) \lambda^{k-1-m_{1}}\left[\varphi_{1}^{*}(0)+\frac{b_{12}^{* 1}}{b_{11}^{* 1}} \varphi_{2}^{*}(0)\right]\right) \\
-\sum_{r=m_{2}+1}^{k-1} \lambda^{k-1-r}\left(\sum_{l=1}^{2} \frac{\left(b_{11}^{* l}\right)^{2}}{b_{12}^{* l}} \lambda^{r-m_{l}}\left[\varphi_{1}^{*}(0)+\frac{b_{12}^{* 1}}{b_{11}^{* 1}} \varphi_{2}^{*}(0)\right]\right. \\
+\sum_{l=3}^{n} \frac{\left(b_{11}^{* l}\right)^{2}}{b_{12}^{* l}}\left[\varphi_{1}^{*}\left(r-m_{l}\right)\right. \\
\left.\left.+\frac{b_{12}^{* 1}}{b_{11}^{* 1}} \varphi_{2}^{*}\left(r-m_{l}\right)\right]\right) \\
=\lambda^{k} \varphi_{2}^{*}(0)-\sum_{r=0}^{k-1} \lambda^{k-1-r}\left(\sum _ { l = 3 } ^ { n } \frac { ( b _ { 1 1 } ^ { * l } ) ^ { 2 } } { b _ { 1 2 } ^ { * l } } \left[\varphi_{1}^{*}\left(r-m_{l}\right)\right.\right. \\
\left.\left.+\frac{b_{12}^{* 1}}{b_{11}^{* 1}} \varphi_{2}^{*}\left(r-m_{l}\right)\right]\right) \\
+\sum_{r=0}^{m_{2}} \lambda^{k-1-r} \frac{\left(b_{11}^{* 2}\right)^{2}}{b_{12}^{* 2}}\left[\varphi_{1}^{*}\left(r-m_{2}\right)+\frac{b_{12}^{* 1}}{b_{11}^{* 1}} \varphi_{2}^{*}\left(r-m_{2}\right)\right] \\
-\frac{\left(b_{11}^{* 1}\right)^{2}}{b_{12}^{* 1}}\left(\sum _ { r = 0 } ^ { m _ { 1 } } \lambda ^ { k - 1 - r } \left[\varphi_{1}^{*}\left(r-m_{1}\right)\right.\right. \\
+\frac{b_{12}^{* 1}}{\left.b_{11}^{* 1} \varphi_{2}^{*}\left(r-m_{1}\right)\right]} \\
\left.\left.m_{1}\right) \lambda^{k-1-m_{1}}\left[\varphi_{1}^{*}(0)+\frac{b_{12}^{* 1}}{b_{11}^{* 1}} \varphi_{2}^{*}(0)\right]\right)
\end{array} \\
& \times\left(\sum_{l=1}^{2} \frac{\left(b_{11}^{* l}\right)^{2}}{b_{12}^{* l}} \lambda^{r-m_{l}}\left[\varphi_{1}^{*}(0)+\frac{b_{12}^{* 1}}{b_{11}^{* 1}} \varphi_{2}^{*}(0)\right]\right. \\
& \begin{array}{r}
\left.+\left(m_{2}-m_{1}\right) \lambda^{k-1-m_{1}}\left[\varphi_{1}^{*}(0)+\frac{b_{12}^{* 1}}{b_{11}^{* 1}} \varphi_{2}^{*}(0)\right]\right) \\
-\sum_{r=m_{2}+1}^{k-1} \lambda^{k-1-r}\left(\sum_{l=1}^{2} \frac{\left(b_{11}^{* l}\right)^{2}}{b_{12}^{* l}} \lambda^{r-m_{l}}\left[\varphi_{1}^{*}(0)+\frac{b_{12}^{* 1}}{b_{11}^{* 1}} \varphi_{2}^{*}(0)\right]\right. \\
+\sum_{l=3}^{n} \frac{\left(b_{11}^{* l}\right)^{2}}{b_{12}^{* l}}\left[\varphi_{1}^{*}\left(r-m_{l}\right)\right. \\
\left.\left.+\frac{b_{12}^{* 1}}{b_{11}^{* 1}} \varphi_{2}^{*}\left(r-m_{l}\right)\right]\right) \\
=\lambda^{k} \varphi_{2}^{*}(0)-\sum_{r=0}^{k-1} \lambda^{k-1-r}\left(\sum _ { l = 3 } ^ { n } \frac { ( b _ { 1 1 } ^ { * l } ) ^ { 2 } } { b _ { 1 2 } ^ { * l } } \left[\varphi_{1}^{*}\left(r-m_{l}\right)\right.\right. \\
\left.\left.+\frac{b_{12}^{* 1}}{b_{11}^{* 1}} \varphi_{2}^{*}\left(r-m_{l}\right)\right]\right) \\
+\sum_{r=0}^{m_{2}} \lambda^{k-1-r} \frac{\left(b_{11}^{* 2}\right)^{2}}{b_{12}^{* 2}}\left[\varphi_{1}^{*}\left(r-m_{2}\right)+\frac{b_{12}^{* 1}}{b_{11}^{* 1}} \varphi_{2}^{*}\left(r-m_{2}\right)\right] \\
-\frac{\left(b_{11}^{* 1}\right)^{2}}{b_{12}^{* 1}}\left(\sum _ { r = 0 } ^ { m _ { 1 } } \lambda ^ { k - 1 - r } \left[\varphi_{1}^{*}\left(r-m_{1}\right)\right.\right. \\
+\frac{b_{12}^{* 1}}{\left.b_{11}^{* 1} \varphi_{2}^{*}\left(r-m_{1}\right)\right]} \\
\left.\left.m_{1}\right) \lambda^{k-1-m_{1}}\left[\varphi_{1}^{*}(0)+\frac{b_{12}^{* 1}}{b_{11}^{* 1}} \varphi_{2}^{*}(0)\right]\right)
\end{array} \\
& \left.+\sum_{l=3}^{n} \frac{\left(b_{11}^{* l}\right)^{2}}{b_{12}^{* l}}\left[\varphi_{1}^{*}\left(r-m_{l}\right)+\frac{b_{12}^{* 1}}{b_{11}^{* 1}} \varphi_{2}^{*}\left(r-m_{l}\right)\right]\right) \\
& \begin{array}{r}
\left.+\left(m_{2}-m_{1}\right) \lambda^{k-1-m_{1}}\left[\varphi_{1}^{*}(0)+\frac{b_{12}^{* 1}}{b_{11}^{* 1}} \varphi_{2}^{*}(0)\right]\right) \\
-\sum_{r=m_{2}+1}^{k-1} \lambda^{k-1-r}\left(\sum_{l=1}^{2} \frac{\left(b_{11}^{* l}\right)^{2}}{b_{12}^{* l}} \lambda^{r-m_{l}}\left[\varphi_{1}^{*}(0)+\frac{b_{12}^{* 1}}{b_{11}^{* 1}} \varphi_{2}^{*}(0)\right]\right. \\
+\sum_{l=3}^{n} \frac{\left(b_{11}^{* l}\right)^{2}}{b_{12}^{* l}}\left[\varphi_{1}^{*}\left(r-m_{l}\right)\right. \\
\left.\left.+\frac{b_{12}^{* 1}}{b_{11}^{* 1}} \varphi_{2}^{*}\left(r-m_{l}\right)\right]\right) \\
=\lambda^{k} \varphi_{2}^{*}(0)-\sum_{r=0}^{k-1} \lambda^{k-1-r}\left(\sum _ { l = 3 } ^ { n } \frac { ( b _ { 1 1 } ^ { * l } ) ^ { 2 } } { b _ { 1 2 } ^ { * l } } \left[\varphi_{1}^{*}\left(r-m_{l}\right)\right.\right. \\
\left.\left.+\frac{b_{12}^{* 1}}{b_{11}^{* 1}} \varphi_{2}^{*}\left(r-m_{l}\right)\right]\right) \\
+\sum_{r=0}^{m_{2}} \lambda^{k-1-r} \frac{\left(b_{11}^{* 2}\right)^{2}}{b_{12}^{* 2}}\left[\varphi_{1}^{*}\left(r-m_{2}\right)+\frac{b_{12}^{* 1}}{b_{11}^{* 1}} \varphi_{2}^{*}\left(r-m_{2}\right)\right] \\
-\frac{\left(b_{11}^{* 1}\right)^{2}}{b_{12}^{* 1}}\left(\sum _ { r = 0 } ^ { m _ { 1 } } \lambda ^ { k - 1 - r } \left[\varphi_{1}^{*}\left(r-m_{1}\right)\right.\right. \\
+\frac{b_{12}^{* 1}}{\left.b_{11}^{* 1} \varphi_{2}^{*}\left(r-m_{1}\right)\right]} \\
\left.\left.m_{1}\right) \lambda^{k-1-m_{1}}\left[\varphi_{1}^{*}(0)+\frac{b_{12}^{* 1}}{b_{11}^{* 1}} \varphi_{2}^{*}(0)\right]\right)
\end{array} \\
& =\lambda^{k-m_{2}-1}\left[\lambda^{m_{2}+1} \varphi_{2}^{*}(0)-\sum_{r=0}^{m_{2}} \lambda^{m_{2}-r}\right. \\
& \times\left(\sum _ { l = 2 } ^ { n } \frac { ( b _ { 1 1 } ^ { * l } ) ^ { 2 } } { b _ { 1 2 } ^ { * l } } \left[\varphi_{1}^{*}\left(r-m_{l}\right)\right.\right. \\
& \left.\left.+\frac{b_{12}^{* 1}}{b_{11}^{* 1}} \varphi_{2}^{*}\left(r-m_{l}\right)\right]\right) \\
& -\frac{\left(b_{11}^{* l}\right)^{2}}{b_{12}^{* 1}}\left(\sum _ { r = 0 } ^ { m _ { 1 } } \lambda ^ { m _ { 2 } - r } \left[\varphi_{1}^{*}\left(r-m_{1}\right)\right.\right. \\
& \left.+\frac{b_{12}^{* 1}}{b_{11}^{* 1}} \varphi_{2}^{*}\left(r-m_{1}\right)\right] \\
& +\left(m_{2}-m_{1}\right) \lambda^{m_{2}-m_{1}} \\
& \left.\left.\times\left[\varphi_{1}^{*}(0)+\frac{b_{12}^{* 1}}{b_{11}^{* 1}} \varphi_{2}^{*}(0)\right]\right)\right] \\
& -\sum_{r=m_{2}+1}^{k-1} \lambda^{k-1-r}\left(\sum_{l=1}^{2} \frac{\left(b_{11}^{* l}\right)^{2}}{b_{12}^{* l}} \lambda^{r-m_{l}}\left[\varphi_{1}^{*}(0)+\frac{b_{12}^{* 1}}{b_{11}^{* 1}} \varphi_{2}^{*}(0)\right]\right. \\
& +\sum_{l=3}^{n} \frac{\left(b_{11}^{* l}\right)^{2}}{b_{12}^{* l}}\left[\varphi_{1}^{*}\left(r-m_{l}\right)\right. \\
& \left.\left.+\frac{b_{12}^{* 1}}{b_{11}^{* 1}} \varphi_{2}^{*}\left(r-m_{l}\right)\right]\right) \\
& -\left(k-1-m_{2}\right) \\
& \times\left(\frac{\left(b_{11}^{* 1}\right)^{2}}{b_{12}^{* 1}} \lambda^{k-1-m_{1}}\left[\varphi_{1}^{*}(0)+\frac{b_{12}^{* 1}}{b_{11}^{* 1}} \varphi_{2}^{*}(0)\right]\right. \\
& \left.+\frac{\left(b_{11}^{* 2}\right)^{2}}{b_{12}^{* 2}} \lambda^{k-1-m_{2}}\left[\varphi_{1}^{*}(0)+\frac{b_{12}^{* 1}}{b_{11}^{* 1}} \varphi_{2}^{*}(0)\right]\right)
\end{aligned}
$$


Abstract and Applied Analysis

19

$$
\begin{gathered}
=\lambda^{k} \varphi_{2}^{*}(0)-\sum_{r=0}^{k-1} \lambda^{k-1-r}\left(\sum _ { l = 3 } ^ { n } \frac { ( b _ { 1 1 } ^ { * l } ) ^ { 2 } } { b _ { 1 2 } ^ { * l } } \left[\varphi_{1}^{*}\left(r-m_{l}\right)\right.\right. \\
\left.\left.+\frac{b_{12}^{* 1}}{b_{11}^{* 1}} \varphi_{2}^{*}\left(r-m_{l}\right)\right]\right) \\
-\frac{\left(b_{11}^{* 1}\right)^{2}}{b_{12}^{* 1}}\left(\sum_{r=0}^{m_{1}} \lambda^{k-1-r}\left[\varphi_{1}^{*}\left(r-m_{1}\right)+\frac{b_{12}^{* 1}}{b_{11}^{* 1}} \varphi_{2}^{*}\left(r-m_{1}\right)\right]\right. \\
+\left(k-1-m_{1}\right) \lambda^{k-1-m_{1}} \\
\times \\
+\frac{\left.b_{11}^{* 2}\right)^{2}}{b_{12}^{* 2}}\left(\sum _ { r = 0 } ^ { m _ { 2 } } \lambda ^ { k - 1 - r } \left[\varphi_{1}^{*}\left(r-m_{2}\right)+\frac{b_{12}^{* 1}}{\left.\left.b_{11}^{* 1} \varphi_{2}^{*}(0)\right]\right)}\right.\right. \\
+\left(k-1-m_{2}\right) \lambda^{k-1-m_{2}} \\
\times\left[\varphi_{1}^{* 1}(0)+\frac{b_{12}^{* 1}}{\left.\left.b_{11}^{* 1} \varphi_{2}^{*}(0)\right]\right) .}\right. \\
\left.+\left(r-m_{2}\right)\right]
\end{gathered}
$$

From (93)-(97) we deduce that expected form of the solution of the initial problem for $k \in \mathbb{Z}_{m_{s-1}+1}^{m_{s}}$ with initial data derived from the solution of previous equation for $k \in$ $\mathbb{Z}_{m_{s-2}+1}^{m_{s-1}}$ is

$y_{1}(k)$

$$
\begin{gathered}
=\lambda^{k} \varphi_{1}^{*}(0)+\sum_{r=0}^{k-1} \lambda^{k-1-r} \\
\times\left(\sum_{l=s}^{n} b_{11}^{* l}\left[\varphi_{1}^{*}\left(r-m_{l}\right)+\frac{b_{12}^{* 1}}{b_{11}^{* 1}} \varphi_{2}^{*}\left(r-m_{l}\right)\right]\right) \\
+\sum_{l=1}^{s-1}\left[b _ { 1 1 } ^ { * l } \left(\sum_{r=0}^{m_{l}} \lambda^{k-1-r}\left[\varphi_{1}^{*}\left(r-m_{l}\right)+\frac{b_{12}^{* 1}}{b_{11}^{* 1}} \varphi_{2}^{*}\left(r-m_{l}\right)\right]\right.\right. \\
+\left(k-1-m_{l}\right) \lambda^{k-1-m_{l}} \\
\left.\left.\times\left[\varphi_{1}^{*}(0)+\frac{b_{12}^{* 1}}{b_{11}^{* 1}} \varphi_{2}^{*}(0)\right]\right)\right] \\
\text { if } k \in \mathbb{Z}_{m_{s-1}+2}^{m_{s}+1}
\end{gathered}
$$

$y_{1}(k)$

$$
\begin{aligned}
= & \lambda^{k} \varphi_{2}^{*}(0)-\sum_{r=0}^{k-1} \lambda^{k-1-r} \\
& \times\left(\sum_{l=s}^{n} \frac{\left(b_{11}^{* l}\right)^{2}}{b_{12}^{* l}}\left[\varphi_{1}^{*}\left(r-m_{l}\right)+\frac{b_{12}^{* 1}}{b_{11}^{* 1}} \varphi_{2}^{*}\left(r-m_{l}\right)\right]\right)
\end{aligned}
$$

$$
\begin{gathered}
-\sum_{l=1}^{s-1}\left[\frac { ( b _ { 1 1 } ^ { * l } ) ^ { 2 } } { b _ { 1 2 } ^ { * l } } \left(\sum_{r=0}^{m_{l}} \lambda^{k-1-r}\left[\varphi_{1}^{*}\left(r-m_{l}\right)+\frac{b_{12}^{* 1}}{b_{11}^{* 1}} \varphi_{2}^{*}\left(r-m_{l}\right)\right]\right.\right. \\
+\left(k-1-m_{l}\right) \lambda^{k-1-m_{l}} \\
\left.\left.\times\left[\varphi_{1}^{*}(0)+\frac{b_{12}^{* 1}}{b_{11}^{* 1}} \varphi_{2}^{*}(0)\right]\right)\right] \\
\text { if } k \in \mathbb{Z}_{m_{s-1}^{m_{s}+1}} .
\end{gathered}
$$

We solve (88) for $k \in \mathbb{Z}_{m_{s}+1}^{m_{s+1}}$ with initial data deduced from (98); that is, we consider the problem

$$
\begin{aligned}
y_{1} & (k+1) \\
= & \lambda y_{1}(k) \\
& +\sum_{l=1}^{s} b_{11}^{* l} \lambda^{k-m_{l}}\left[\varphi_{1}^{*}(0)+\frac{b_{12}^{* 1}}{b_{11}^{* 1}} \varphi_{2}^{*}(0)\right] \\
& +\sum_{l=s+1}^{n} b_{11}^{* l}\left[\varphi_{1}^{*}\left(k-m_{l}\right)+\frac{b_{12}^{* 1}}{b_{11}^{* 1}} \varphi_{2}^{*}\left(k-m_{l}\right)\right] \\
y_{1} & \left(m_{s}+1\right) \text { if } k \in \mathbb{Z}_{m_{s}+1}^{m_{s+1}}, \\
= & \lambda^{m_{s}+1} \varphi_{1}^{*}(0) \\
& +\sum_{r=0}^{m_{s}} \lambda^{m_{s}-r}\left(\sum_{l=s}^{n} b_{11}^{* l}\left[\varphi_{1}^{*}\left(r-m_{l}\right)+\frac{b_{12}^{* 1}}{b_{11}^{* 1}} \varphi_{2}^{*}\left(r-m_{l}\right)\right]\right) \\
& +\sum_{l=s+1}^{n} \frac{\left(b_{11}^{* l}\right)^{2}}{b_{12}^{* l}}\left[\varphi_{1}^{*}\left(k-m_{l}\right)+\frac{b_{12}^{* 1}}{b_{11}^{* 1}} \varphi_{2}^{*}\left(k-m_{l}\right)\right] \\
& +\sum_{l=1} b_{11}^{* l}\left(\sum_{r=0}^{m_{l}} \lambda^{m_{s}-r}\left[\varphi_{1}^{*}\left(r-m_{l}\right)+\frac{b_{12}^{* 1}}{b_{11}^{* 1}} \varphi_{2}^{*}\left(r-m_{l}\right)\right]\right. \\
y_{2} & (k+1) \\
& \left.\left.+\varphi_{1}^{*}(0)+\frac{b_{12}^{* 1}}{b_{11}^{* 1}} \varphi_{2}^{*}(0)\right]\right)
\end{aligned}
$$


20

Abstract and Applied Analysis

$$
\begin{aligned}
& y_{2}\left(m_{s}+1\right) \\
& =\lambda^{m_{s}+1} \varphi_{2}^{*}(0) \\
& -\sum_{r=0}^{m_{s}} \lambda^{m_{s}-r}\left(\sum_{l=s}^{n} \frac{\left(b_{11}^{* l}\right)^{2}}{b_{12}^{* l}}\left[\varphi_{1}^{*}\left(r-m_{l}\right)+\frac{b_{12}^{* 1}}{b_{11}^{* 1}} \varphi_{2}^{*}\left(r-m_{l}\right)\right]\right) \\
& -\sum_{l=1}^{s-1} \frac{\left(b_{11}^{* l}\right)^{2}}{b_{12}^{* l}}\left(\sum_{r=0}^{m_{l}} \lambda^{m_{s}-r}\left[\varphi_{1}^{*}\left(r-m_{l}\right)+\frac{b_{12}^{* 1}}{b_{11}^{* 1}} \varphi_{2}^{*}\left(r-m_{l}\right)\right]\right. \\
& \left.\quad+\left(m_{s}-m_{l}\right) \lambda^{m_{s}-m_{l}}\left[\varphi_{1}^{*}(0)+\frac{b_{12}^{* 1}}{b_{11}^{* 1}} \varphi_{2}^{*}(0)\right]\right) .
\end{aligned}
$$

Applying formula (33) yields (for $k \in \mathbb{Z}_{m_{s}+2}^{m_{s+1}+1}$ )

$$
\begin{aligned}
& y_{1}(k) \\
& =\lambda^{k-\left(m_{s}+1\right)} y_{1}\left(m_{s}+1\right)+\sum_{r=m_{s}+1}^{k-1} \lambda^{k-1-r} \\
& \times\left(\sum _ { l = 1 } ^ { s } b _ { 1 1 } ^ { * l } \lambda ^ { r - m _ { l } } \left[\varphi_{1}^{*}(0)+\frac{b_{12}^{* 1}}{\left.b_{11}^{* 1} \varphi_{2}^{*}(0)\right]}\right.\right. \\
& \left.+\sum_{l=s+1}^{n} b_{11}^{* l}\left[\varphi_{1}^{*}\left(r-m_{l}\right)+\frac{b_{12}^{* 1}}{b_{11}^{* 1}} \varphi_{2}^{*}\left(r-m_{l}\right)\right]\right) \\
& =\lambda^{k-m_{s}-1}\left[\lambda^{m_{s}+1} \varphi_{1}^{*}(0)+\sum_{r=0}^{m_{s}} \lambda_{s}^{m_{s}-r}\right. \\
& \times\left(\sum_{l=s}^{n} b_{11}^{* l}\left[\varphi_{1}^{*}\left(r-m_{l}\right)+\frac{b_{12}^{* 1}}{b_{11}^{* 1}} \varphi_{2}^{*}\left(r-m_{l}\right)\right]\right) \\
& +\sum_{l=1}^{s-1} b_{11}^{* l}\left(\sum _ { r = 0 } ^ { m _ { l } } \lambda ^ { m _ { s } - r } \left[\varphi_{1}^{*}\left(r-m_{l}\right)\right.\right. \\
& +\left(m_{s}-m_{l}\right) \lambda^{m_{s}-m_{l}} \\
& \left.\left.\times\left[\varphi_{12}^{*}(0)+\frac{b_{12}^{* 1}}{b_{11}^{* 1}} \varphi_{2}^{*}(0)\right]\right)\right] \\
& +\sum_{r=m_{s}+1}^{k-1} \lambda^{k-1-r}\left(\sum_{l=1}^{s} b_{11}^{* l} \lambda^{r-m_{l}}\left[\varphi_{1}^{*}(0)+\frac{b_{12}^{* 1}}{b_{11}^{* 1}} \varphi_{2}^{*}(0)\right]\right. \\
& \left.\left.b_{11}^{* 1} \varphi_{2}^{*}\left(r-m_{l}\right)\right]\right)
\end{aligned}
$$

$$
=\lambda^{k} \varphi_{1}^{*}(0)+\sum_{r=0}^{m_{s}} \lambda^{k-1-r}
$$

$$
\begin{gathered}
\times\left(\sum_{l=s}^{n} b_{11}^{* l}\left[\varphi_{1}^{*}\left(r-m_{l}\right)+\frac{b_{12}^{* 1}}{b_{11}^{* 1}} \varphi_{2}^{*}\left(r-m_{l}\right)\right]\right) \\
+\sum_{l=1}^{s-1} b_{11}^{* l}\left(\sum_{r=0}^{m_{l}} \lambda^{k-1-r}\left[\varphi_{1}^{*}\left(r-m_{l}\right)+\frac{b_{12}^{* 1}}{b_{11}^{* 1}} \varphi_{2}^{*}\left(r-m_{l}\right)\right]\right. \\
+\left(m_{s}-m_{l}\right) \lambda^{k-1-m_{l}} \\
\left.\times\left[\varphi_{1}^{*}(0)+\frac{b_{12}^{* 1}}{b_{11}^{* 1}} \varphi_{2}^{*}(0)\right]\right) \\
+\sum_{r=m_{s}+1}^{k-1} \lambda^{k-1-r}\left(\sum_{l=1}^{s} b_{11}^{* l} \lambda^{r-m_{l}}\left[\varphi_{1}^{*}(0)+\frac{b_{12}^{* 1}}{b_{11}^{* 1}} \varphi_{2}^{*}(0)\right]\right. \\
+\sum_{l=s+1}^{n} b_{11}^{* l}\left[\varphi_{1}^{*}\left(r-m_{l}\right)\right. \\
+\frac{b_{12}^{* 1}}{\left.\left.b_{11}^{*} \varphi_{2}^{*}\left(r-m_{l}\right)\right]\right)}
\end{gathered}
$$

$$
\begin{aligned}
= & \lambda^{k} \varphi_{1}^{*}(0)+\sum_{r=0}^{k-1} \lambda^{k-1-r} \\
& \times\left(\sum_{l=s+1}^{n} b_{11}^{* l}\left[\varphi_{1}^{*}\left(r-m_{l}\right)+\frac{b_{12}^{* 1}}{b_{11}^{* 1}} \varphi_{2}^{*}\left(r-m_{l}\right)\right]\right) \\
& +b_{11}^{* s} \sum_{r=0}^{m_{s}} \lambda^{k-1-r}\left[\varphi_{1}^{*}\left(r-m_{s}\right)+\frac{b_{12}^{* 1}}{b_{11}^{* 1}} \varphi_{2}^{*}\left(r-m_{s}\right)\right] \\
& +b_{11}^{* 1}\left(\sum_{r=0}^{m_{1}} \lambda^{k-1-r}\left[\varphi_{1}^{*}\left(r-m_{1}\right)+\frac{b_{12}^{* 1}}{b_{11}^{* 1}} \varphi_{2}^{*}\left(r-m_{1}\right)\right]\right.
\end{aligned}
$$$$
\left.+\left(m_{s}-m_{1}\right) \lambda^{k-1-m_{1}}\left[\varphi_{1}^{*}(0)+\frac{b_{12}^{* 1}}{b_{11}^{* 1}} \varphi_{2}^{*}(0)\right]\right)
$$$$
+b_{11}^{* 2}\left(\sum_{r=0}^{m_{2}} \lambda^{k-1-r}\left[\varphi_{1}^{*}\left(r-m_{2}\right)+\frac{b_{12}^{* 1}}{b_{11}^{* 1}} \varphi_{2}^{*}\left(r-m_{2}\right)\right]\right.
$$$$
+\left(m_{s}-m_{2}\right) \lambda^{k-1-m_{2}}
$$$$
\left.\times\left[\varphi_{1}^{*}(0)+\frac{b_{12}^{* 1}}{b_{11}^{* 1}} \varphi_{2}^{*}(0)\right]\right)
$$

$+\cdots$

$$
+b_{11}^{* s-1}\left(\sum _ { r = 0 } ^ { m _ { s - 1 } } \lambda ^ { k - 1 - r } \left[\varphi_{1}^{*}\left(r-m_{s-1}\right)\right.\right.
$$

$$
\left.+\frac{b_{12}^{* 1}}{b_{11}^{* 1}} \varphi_{2}^{*}\left(r-m_{s-1}\right)\right]
$$


Abstract and Applied Analysis

21

$$
\begin{aligned}
& +\left(m_{s}-m_{s-1}\right) \lambda^{k-1-m_{s-1}} \\
& \left.\times\left[\varphi_{1}^{*}(0)+\frac{b_{12}^{* 1}}{b_{11}^{* 1}} \varphi_{2}^{*}(0)\right]\right) \\
& +b_{11}^{* s}\left(\sum_{r=0}^{m_{s}} \lambda^{k-1-r}\left[\varphi_{1}^{*}\left(r-m_{s}\right)+\frac{b_{12}^{* 1}}{b_{11}^{* 1}} \varphi_{2}^{*}\left(r-m_{s}\right)\right]\right. \\
& \left.+\left(k-1-m_{s}\right) \lambda^{k-1-m_{s}}\left[\varphi_{1}^{*}(0)+\frac{b_{12}^{* 1}}{b_{11}^{* 1}} \varphi_{2}^{*}(0)\right]\right) \\
& +\left(k-1-m_{s}\right) \\
& \times\left(\lambda^{k-1-m_{1}} b_{11}^{* 1}\left[\varphi_{1}^{*}(0)+\frac{b_{12}^{* 1}}{b_{11}^{* 1}} \varphi_{2}^{*}(0)\right]\right. \\
& +\lambda^{k-1-m_{2}} b_{11}^{* 2}\left[\varphi_{1}^{*}(0)+\frac{b_{12}^{* 1}}{b_{11}^{* 1}} \varphi_{2}^{*}(0)\right]+\cdots \\
& +\lambda^{k-1-m_{s-1}} b_{11}^{* s-1}\left[\varphi_{1}^{*}(0)+\frac{b_{12}^{* 1}}{b_{11}^{* 1}} \varphi_{2}^{*}(0)\right] \\
& \left.+\lambda^{k-1-m_{s}} b_{11}^{* s}\left[\varphi_{1}^{*}(0)+\frac{b_{12}^{* 1}}{b_{11}^{* *}} \varphi_{2}^{*}(0)\right]\right) \\
& =\lambda^{k} \varphi_{1}^{*}(0)+\sum_{r=0}^{k-1} \lambda^{k-1-r} \\
& \times\left(\sum_{l=s+1}^{n} b_{11}^{* l}\left[\varphi_{1}^{*}\left(r-m_{l}\right)+\frac{b_{12}^{* 1}}{b_{11}^{* 1}} \varphi_{2}^{*}\left(r-m_{l}\right)\right]\right) \\
& +b_{11}^{* 1}\left(\sum_{r=0}^{m_{1}} \lambda^{k-1-r}\left[\varphi_{1}^{*}\left(r-m_{1}\right)+\frac{b_{12}^{* 1}}{b_{11}^{* 1}} \varphi_{2}^{*}\left(r-m_{1}\right)\right]\right. \\
& +\left(k-1-m_{1}\right) \lambda^{k-1-m_{1}} \\
& \left.\times\left[\varphi_{1}^{*}(0)+\frac{b_{12}^{* 1}}{b_{11}^{* 1}} \varphi_{2}^{*}(0)\right]\right) \\
& +b_{11}^{* 2}\left(\sum_{r=0}^{m_{2}} \lambda^{k-1-r}\left[\varphi_{1}^{*}\left(r-m_{2}\right)+\frac{b_{12}^{* 1}}{b_{11}^{* 1}} \varphi_{2}^{*}\left(r-m_{2}\right)\right]\right. \\
& +\left(k-1-m_{2}\right) \lambda^{k-1-m_{2}} \\
& \left.\times\left[\varphi_{1}^{*}(0)+\frac{b_{12}^{* 1}}{b_{11}^{* 1}} \varphi_{2}^{*}(0)\right]\right) \\
& +\cdots \\
& +b_{11}^{* s-1}\left(\sum _ { r = 0 } ^ { m _ { s - 1 } } \lambda ^ { k - 1 - r } \left[\varphi_{1}^{*}\left(r-m_{s-1}\right)\right.\right. \\
& \left.+\frac{b_{12}^{* 1}}{b_{11}^{* 1}} \varphi_{2}^{*}\left(r-m_{s-1}\right)\right] \\
& +\left(k-1-m_{s-1}\right) \lambda^{k-1-m_{s-1}} \\
& \left.\times\left[\varphi_{1}^{*}(0)+\frac{b_{12}^{* 1}}{b_{11}^{* 1}} \varphi_{2}^{*}(0)\right]\right) \\
& y_{2}(k) \\
& =\lambda^{k-\left(m_{s}+1\right)} y_{2}\left(m_{s}+1\right)-\sum_{r=m_{s}+1}^{k-1} \lambda^{k-1-r} \\
& \times\left(\sum_{l=1}^{s} \frac{\left(b_{11}^{* l}\right)^{2}}{b_{12}^{* l}} \lambda^{r-m_{l}}\left[\varphi_{1}^{*}(0)+\frac{b_{12}^{* 1}}{b_{11}^{* 1}} \varphi_{2}^{*}(0)\right]\right. \\
& +\sum_{l=s+1}^{n} \frac{\left(b_{11}^{* l}\right)^{2}}{b_{12}^{* l}}\left[\varphi_{1}^{*}\left(r-m_{l}\right)\right. \\
& \left.\left.+\frac{b_{12}^{* 1}}{b_{11}^{* 1}} \varphi_{2}^{*}\left(r-m_{l}\right)\right]\right) \\
& =\lambda^{k-m_{s}-1}\left[\lambda^{m_{s}+1} \varphi_{2}^{*}(0)-\sum_{r=0}^{m_{s}} \lambda^{m_{s}-r}\right. \\
& \times\left(\sum _ { l = s } ^ { n } \frac { ( b _ { 1 1 } ^ { * l } ) ^ { 2 } } { b _ { 1 2 } ^ { * l } } \left[\varphi_{1}^{*}\left(r-m_{l}\right)\right.\right. \\
& \left.\left.+\frac{b_{12}^{* 1}}{b_{11}^{* 1}} \varphi_{2}^{*}\left(r-m_{l}\right)\right]\right) \\
& -\sum_{l=1}^{s-1} \frac{\left(b_{11}^{* l}\right)^{2}}{b_{12}^{* l}}\left(\sum _ { r = 0 } ^ { m _ { l } } \lambda ^ { m _ { s } - r } \left[\varphi_{1}^{*}\left(r-m_{l}\right)\right.\right. \\
& \left.+\frac{b_{12}^{* 1}}{b_{11}^{* 1}} \varphi_{2}^{*}\left(r-m_{l}\right)\right]
\end{aligned}
$$

(100) 
22

Abstract and Applied Analysis

$$
\begin{aligned}
& +\left(m_{s}-m_{l}\right) \lambda^{m_{s}-m_{l}} \\
& \left.\left.\times\left[\varphi_{1}^{*}(0)+\frac{b_{12}^{* 1}}{b_{11}^{* 1}} \varphi_{2}^{*}(0)\right]\right)\right] \\
& -\frac{\left(b_{11}^{* 1}\right)^{2}}{b_{12}^{* 1}}\left(\sum_{r=0}^{m_{1}} \lambda^{k-1-r}\left[\varphi_{1}^{*}\left(r-m_{1}\right)+\frac{b_{12}^{* 1}}{b_{11}^{* 1}} \varphi_{2}^{*}\left(r-m_{1}\right)\right]\right. \\
& \left.+\left(m_{s}-m_{1}\right) \lambda^{k-1-m_{1}}\left[\varphi_{1}^{*}(0)+\frac{b_{12}^{* 1}}{b_{11}^{* 1}} \varphi_{2}^{*}(0)\right]\right) \\
& -\sum_{r=m_{s}+1}^{k-1} \lambda^{k-1-r}\left(\sum_{l=1}^{s} \frac{\left(b_{11}^{* l}\right)^{2}}{b_{12}^{* l}} \lambda^{r-m_{l}}\left[\varphi_{1}^{*}(0)+\frac{b_{12}^{* 1}}{b_{11}^{* 1}} \varphi_{2}^{*}(0)\right]\right. \\
& +\sum_{l=s+1}^{n} \frac{\left(b_{11}^{* l}\right)^{2}}{b_{12}^{* l}}\left[\varphi_{1}^{*}\left(r-m_{l}\right)\right. \\
& \left.\left.+\frac{b_{12}^{* 1}}{b_{11}^{* 1}} \varphi_{2}^{*}\left(r-m_{l}\right)\right]\right) \\
& =\lambda^{k} \varphi_{2}^{*}(0)-\sum_{r=0}^{m_{s}} \lambda^{k-1-r} \\
& -\frac{\left(b_{11}^{* 2}\right)^{2}}{b_{12}^{* 2}}\left(\sum_{r=0}^{m_{2}} \lambda^{k-1-r}\left[\varphi_{1}^{*}\left(r-m_{2}\right)+\frac{b_{12}^{* 1}}{b_{11}^{* 1}} \varphi_{2}^{*}\left(r-m_{2}\right)\right]\right. \\
& \left.+\left(m_{s}-m_{2}\right) \lambda^{k-1-m_{2}}\left[\varphi_{1}^{*}(0)+\frac{b_{12}^{* 1}}{b_{11}^{* 1}} \varphi_{2}^{*}(0)\right]\right) \\
& +\cdots \\
& \times\left(\sum_{l=s}^{n} \frac{\left(b_{11}^{* l}\right)^{2}}{b_{12}^{* l}}\left[\varphi_{1}^{*}\left(r-m_{l}\right)+\frac{b_{12}^{* 1}}{b_{11}^{* 1}} \varphi_{2}^{*}\left(r-m_{l}\right)\right]\right) \\
& -\frac{\left(b_{11}^{* s-1}\right)^{2}}{b_{12}^{* s-1}}\left(\sum_{r=0}^{m_{s-1}} \lambda^{k-1-r}\left[\varphi_{1}^{*}\left(r-m_{s-1}\right)+\frac{b_{12}^{* 1}}{b_{11}^{* 1}} \varphi_{2}^{*}\left(r-m_{s-1}\right)\right]\right. \\
& \left.+\left(m_{s}-m_{s-1}\right) \lambda^{k-1-m_{s-1}}\left[\varphi_{1}^{*}(0)+\frac{b_{12}^{* 1}}{b_{11}^{* 1}} \varphi_{2}^{*}(0)\right]\right) \\
& -\sum_{l=1}^{s-1} \frac{\left(b_{11}^{* l}\right)^{2}}{b_{12}^{* l}}\left(\sum _ { r = 0 } ^ { m _ { l } } \lambda ^ { k - 1 - r } \left[\varphi_{1}^{*}\left(r-m_{l}\right)\right.\right. \\
& \left.+\frac{b_{12}^{* 1}}{b_{11}^{* 1}} \varphi_{2}^{*}\left(r-m_{l}\right)\right] \\
& \left.+\left(m_{s}-m_{l}\right) \lambda^{k-1-m_{l}}\left[\varphi_{1}^{*}(0)+\frac{b_{12}^{* 1}}{b_{11}^{* 1}} \varphi_{2}^{*}(0)\right]\right) \\
& -\sum_{r=m_{s}+1}^{k-1} \lambda^{k-1-r}\left(\sum_{l=1}^{s} \frac{\left(b_{11}^{* l}\right)^{2}}{b_{12}^{* l}} \lambda^{r-m_{l}}\left[\varphi_{1}^{*}(0)+\frac{b_{12}^{* 1}}{b_{11}^{* 1}} \varphi_{2}^{*}(0)\right]\right. \\
& +\sum_{l=s+1}^{n} \frac{\left(b_{11}^{* l}\right)^{2}}{b_{12}^{* l}}\left[\varphi_{1}^{*}\left(r-m_{l}\right)\right. \\
& \left.\left.+\frac{b_{12}^{* 1}}{b_{11}^{* 1}} \varphi_{2}^{*}\left(r-m_{l}\right)\right]\right) \\
& =\lambda^{k} \varphi_{2}^{*}(0)-\sum_{r=0}^{k-1} \lambda^{k-1-r} \\
& \times\left(\sum_{l=s+1}^{n} \frac{\left(b_{11}^{* l}\right)^{2}}{b_{12}^{* l}}\left[\varphi_{1}^{*}\left(r-m_{l}\right)+\frac{b_{12}^{* 1}}{b_{11}^{* 1}} \varphi_{2}^{*}\left(r-m_{l}\right)\right]\right) \\
& -\frac{\left(b_{11}^{* s}\right)^{2}}{b_{12}^{* s}} \sum_{r=0}^{m_{s}} \lambda^{k-1-r}\left[\varphi_{1}^{*}\left(r-m_{s}\right)+\frac{b_{12}^{* 1}}{b_{11}^{* 1}} \varphi_{2}^{*}\left(r-m_{s}\right)\right] \\
& -\left(k-1-m_{s}\right) \\
& \times\left(\lambda^{k-1-m_{1}} \frac{\left(b_{11}^{* 1}\right)^{2}}{b_{12}^{* 1}}\left[\varphi_{1}^{*}(0)+\frac{b_{12}^{* 1}}{b_{11}^{* 1}} \varphi_{2}^{*}(0)\right]\right. \\
& +\lambda^{k-1-m_{2}} \frac{\left(b_{11}^{* 2}\right)^{2}}{b_{12}^{* 2}}\left[\varphi_{1}^{*}(0)+\frac{b_{12}^{* 1}}{b_{11}^{* 1}} \varphi_{2}^{*}(0)\right]+\cdots \\
& +\lambda^{k-1-m_{s-1}} \frac{\left(b_{11}^{* s-1}\right)^{2}}{b_{12}^{* s-1}}\left[\varphi_{1}^{*}(0)+\frac{b_{12}^{* 1}}{b_{11}^{* 1}} \varphi_{2}^{*}(0)\right] \\
& \left.+\lambda^{k-1-m_{s}} \frac{\left(b_{11}^{* s}\right)^{2}}{b_{12}^{* s}}\left[\varphi_{1}^{*}(0)+\frac{b_{12}^{* 1}}{b_{11}^{* 1}} \varphi_{2}^{*}(0)\right]\right) \\
& =\lambda^{k} \varphi_{2}^{*}(0)-\sum_{r=0}^{k-1} \lambda^{k-1-r} \\
& \times\left(\sum_{l=s+1}^{n} \frac{\left(b_{11}^{* l}\right)^{2}}{b_{12}^{* l}}\left[\varphi_{1}^{*}\left(r-m_{l}\right)+\frac{b_{12}^{* 1}}{b_{11}^{* 1}} \varphi_{2}^{*}\left(r-m_{l}\right)\right]\right) \\
& -\frac{\left(b_{11}^{* 1}\right)^{2}}{b_{12}^{* 1}}\left(\sum_{r=0}^{m_{1}} \lambda^{k-1-r}\left[\varphi_{1}^{*}\left(r-m_{1}\right)+\frac{b_{12}^{* 1}}{b_{11}^{* 1}} \varphi_{2}^{*}\left(r-m_{1}\right)\right]\right. \\
& +\left(k-1-m_{1}\right) \lambda^{k-1-m_{1}} \\
& \left.\times\left[\varphi_{1}^{*}(0)+\frac{b_{12}^{* 1}}{b_{11}^{* 1}} \varphi_{2}^{*}(0)\right]\right)
\end{aligned}
$$




$$
\begin{aligned}
& -\frac{\left(b_{11}^{* 2}\right)^{2}}{b_{12}^{* 2}}\left(\sum_{r=0}^{m_{2}} \lambda^{k-1-r}\left[\varphi_{1}^{*}\left(r-m_{2}\right)+\frac{b_{12}^{* 1}}{b_{11}^{* 1}} \varphi_{2}^{*}\left(r-m_{2}\right)\right]\right. \\
& +\left(k-1-m_{2}\right) \lambda^{k-1-m_{2}} \\
& \left.\times\left[\varphi_{1}^{*}(0)+\frac{b_{12}^{* 1}}{b_{11}^{* 1}} \varphi_{2}^{*}(0)\right]\right) \\
& y_{1}(k+1)=\lambda y_{1}(k)+\sum_{l=1}^{n} b_{11}^{* l} \lambda^{k-m_{l}}\left[\varphi_{1}^{*}(0)+\frac{b_{12}^{* 1}}{b_{11}^{* 1}} \varphi_{2}^{*}(0)\right] \\
& \text { if } k \in \mathbb{Z}_{m_{n}+1}^{\infty} \text {, } \\
& +\cdots \\
& -\frac{\left(b_{11}^{* s-1}\right)^{2}}{b_{12}^{* s-1}}\left(\sum _ { r = 0 } ^ { m _ { s - 1 } } \lambda ^ { k - 1 - r } \left[\varphi_{1}^{*}\left(r-m_{s-1}\right)\right.\right. \\
& \left.+\frac{b_{12}^{* 1}}{b_{11}^{* 1}} \varphi_{2}^{*}\left(r-m_{s-1}\right)\right] \\
& +\left(k-1-m_{s-1}\right) \lambda^{k-1-m_{s-1}} \\
& \left.\times\left[\varphi_{1}^{*}(0)+\frac{b_{12}^{* 1}}{b_{11}^{* 1}} \varphi_{2}^{*}(0)\right]\right) \\
& -\frac{\left(b_{11}^{* s}\right)^{2}}{b_{12}^{* s}}\left(\sum _ { r = 0 } ^ { m _ { s } } \lambda ^ { k - 1 - r } \left[\varphi_{1}^{*}\left(r-m_{s}\right)\right.\right. \\
& \left.+\frac{b_{12}^{* 1}}{b_{11}^{* 1}} \varphi_{2}^{*}\left(r-m_{s}\right)\right] \\
& +\left(k-1-m_{s}\right) \lambda^{k-1-m_{s}} \\
& \left.\times\left[\varphi_{1}^{*}(0)+\frac{b_{12}^{* 1}}{b_{11}^{* 1}} \varphi_{2}^{*}(0)\right]\right) \\
& =\lambda^{k} \varphi_{2}^{*}(0)-\sum_{r=0}^{k-1} \lambda^{k-1-r} \\
& \times\left(\sum_{l=s+1}^{n} \frac{\left(b_{11}^{* l}\right)^{2}}{b_{12}^{* l}}\left[\varphi_{1}^{*}\left(r-m_{l}\right)+\frac{b_{12}^{* 1}}{b_{11}^{* 1}} \varphi_{2}^{*}\left(r-m_{l}\right)\right]\right) \\
& -\sum_{l=1}^{s} \frac{\left(b_{11}^{* l}\right)^{2}}{b_{12}^{* l}}\left(\sum _ { r = 0 } ^ { m _ { l } } \lambda ^ { k - 1 - r } \left[\varphi_{1}^{*}\left(r-m_{l}\right)\right.\right. \\
& \left.+\frac{b_{12}^{* 1}}{b_{11}^{* 1}} \varphi_{2}^{*}\left(r-m_{l}\right)\right] \\
& +\left(k-1-m_{l}\right) \lambda^{k-1-m_{l}} \\
& \left.\times\left[\varphi_{1}^{*}(0)+\frac{b_{12}^{* 1}}{b_{11}^{* 1}} \varphi_{2}^{*}(0)\right]\right) \text {. } \\
& y_{1}\left(m_{n}+1\right) \\
& =\lambda^{m_{n}+1} \varphi_{1}^{*}(0)+\sum_{r=0}^{m_{n}} \lambda^{m_{n}-r} b_{11}^{* n} \\
& \times\left[\varphi_{1}^{*}\left(r-m_{n}\right)+\frac{b_{12}^{* 1}}{b_{11}^{* 1}} \varphi_{2}^{*}\left(r-m_{n}\right)\right] \\
& +\sum_{l=1}^{n-1} b_{11}^{* l}\left(\sum _ { r = 0 } ^ { m _ { l } } \lambda ^ { m _ { n } - r } \left[\varphi_{1}^{*}\left(r-m_{l}\right)\right.\right. \\
& \left.+\frac{b_{12}^{* 1}}{b_{11}^{* 1}} \varphi_{2}^{*}\left(r-m_{l}\right)\right] \\
& +\left(m_{n}-m_{l}\right) \lambda^{m_{n}-m_{l}} \\
& \left.\times\left[\varphi_{1}^{*}(0)+\frac{b_{12}^{* 1}}{b_{11}^{* 1}} \varphi_{2}^{*}(0)\right]\right), \\
& y_{2}(k+1) \\
& \begin{aligned}
= & \lambda y_{2}(k)-\sum_{l=1}^{n} \frac{\left(b_{11}^{* l}\right)^{2}}{b_{12}^{* l}} \lambda^{k-m_{l}} \\
& \times\left[\varphi_{1}^{*}(0)+\frac{b_{12}^{* 1}}{b_{11}^{* 1}} \varphi_{2}^{*}(0)\right] \quad \text { if } k \in \mathbb{Z}_{m_{n}+1}^{\infty},
\end{aligned} \\
& y_{2}\left(m_{n}+1\right) \\
& =\lambda^{m_{n}+1} \varphi_{2}^{*}(0)-\sum_{r=0}^{m_{n}} \lambda^{m_{n}-r} \frac{\left(b_{11}^{* n}\right)^{2}}{b_{12}^{* n}} \\
& \times\left[\varphi_{1}^{*}\left(r-m_{n}\right)+\frac{b_{12}^{* 1}}{b_{11}^{* 1}} \varphi_{2}^{*}\left(r-m_{n}\right)\right] \\
& -\sum_{l=1}^{n-1} \frac{\left(b_{11}^{* l}\right)^{2}}{b_{12}^{* l}}\left(\sum _ { r = 0 } ^ { m _ { l } } \lambda ^ { m _ { n } - r } \left[\varphi_{1}^{*}\left(r-m_{l}\right)\right.\right. \\
& \left.+\frac{b_{12}^{* 1}}{b_{11}^{* 1}} \varphi_{2}^{*}\left(r-m_{l}\right)\right] \\
& +\left(m_{n}-m_{l}\right) \lambda^{m_{n}-m_{l}} \\
& \left.\times\left[\varphi_{1}^{*}(0)+\frac{b_{12}^{* 1}}{b_{11}^{* 1}} \varphi_{2}^{*}(0)\right]\right) .
\end{aligned}
$$


Applying formula (33) yields (for $k \in \mathbb{Z}_{m_{n}+2}^{\infty}$ )

$$
\begin{aligned}
& +\sum_{r=m_{n}+1}^{k-1} \lambda^{k-1-r}\left(\sum_{l=1}^{n} b_{11}^{* l} \lambda^{r-m_{l}}\right. \\
& \left.\times\left[\varphi_{1}^{*}(0)+\frac{b_{12}^{* 1}}{b_{11}^{* 1}} \varphi_{2}^{*}(0)\right]\right) \\
& =\lambda^{k} \varphi_{1}^{*}(0)+\sum_{r=0}^{m_{n}} \lambda^{k-1-r} b_{11}^{* n}\left[\varphi_{1}^{*}\left(r-m_{n}\right)\right. \\
& \left.+\frac{b_{12}^{* 1}}{b_{11}^{* 1}} \varphi_{2}^{*}\left(r-m_{n}\right)\right] \\
& +b_{11}^{* 1}\left(\sum _ { r = 0 } ^ { m _ { 1 } } \lambda ^ { k - 1 - r } \left[\varphi_{1}^{*}\left(r-m_{1}\right)+\frac{b_{12}^{* 1}}{\left.b_{11}^{* 1} \varphi_{2}^{*}\left(r-m_{1}\right)\right]}\right.\right. \\
& +\left(m_{n}-m_{1}\right) \lambda^{k-1-m_{1}} \\
& \left.\quad \times\left[\varphi_{1}^{*}(0)+\frac{b_{12}^{* 1}}{b_{11}^{*}} \varphi_{2}^{*}(0)\right]\right) \\
& +b_{11}^{* 2}\left(\sum_{r=0}^{m_{2}} \lambda^{k-1-r}\left[\varphi_{1}^{*}\left(r-m_{2}\right)+\frac{b_{12}^{* 1}}{b_{11}^{* 1}} \varphi_{2}^{*}\left(r-m_{2}\right)\right]\right. \\
& +\left(m_{n}-m_{2}\right) \lambda^{k-1-m_{2}} \\
& \left.\times\left[\varphi_{1}^{*}(0)+\frac{b_{12}^{* 1}}{b_{11}^{* 1}} \varphi_{2}^{*}(0)\right]\right) \\
& +\cdots \quad
\end{aligned}
$$$$
y_{1}(k)
$$$$
=\lambda^{k-\left(m_{n}+1\right)} y_{1}\left(m_{n}+1\right)+\sum_{r=m_{n}+1}^{k-1} \lambda^{k-1-r}
$$$$
\times\left(\sum_{l=1}^{n} b_{11}^{* l} \lambda^{r-m_{l}}\left[\varphi_{1}^{*}(0)+\frac{b_{12}^{* 1}}{b_{11}^{* 1}} \varphi_{2}^{*}(0)\right]\right)
$$$$
=\lambda^{k-m_{n}-1}\left[\lambda^{m_{n}+1} \varphi_{1}^{*}(0)\right.
$$$$
+\sum_{r=0}^{m_{n}} \lambda^{m_{n}-r} b_{11}^{* n}\left[\varphi_{1}^{*}\left(r-m_{n}\right)\right.
$$$$
\left.+\frac{b_{12}^{* 1}}{b_{11}^{* 1}} \varphi_{2}^{*}\left(r-m_{n}\right)\right]
$$$$
+\sum_{l=1}^{n-1} b_{11}^{* l}\left(\sum _ { r = 0 } ^ { m _ { l } } \lambda ^ { m _ { n } - r } \left[\varphi_{1}^{*}\left(r-m_{l}\right)\right.\right.
$$$$
\left.+\frac{b_{12}^{* 1}}{b_{11}^{* 1}} \varphi_{2}^{*}\left(r-m_{l}\right)\right]
$$$$
+\left(m_{n}-m_{l}\right) \lambda^{m_{n}-m_{l}}
$$$$
\left.\left.\times\left[\varphi_{1}^{*}(0)+\frac{b_{12}^{* 1}}{b_{11}^{* 1}} \varphi_{2}^{*}(0)\right]\right)\right]
$$$$
+\sum_{r=m_{n}+1}^{k-1} \lambda^{k-1-r}\left(\sum_{l=1}^{n} b_{11}^{* l} \lambda^{r-m_{l}}\right.
$$$$
\left.\times\left[\varphi_{1}^{*}(0)+\frac{b_{12}^{* 1}}{b_{11}^{* 1}} \varphi_{2}^{*}(0)\right]\right)
$$$$
=\lambda^{k} \varphi_{1}^{*}(0)+\sum_{r=0}^{m_{n}} \lambda^{k-1-r} b_{11}^{* n}\left[\varphi_{1}^{*}\left(r-m_{n}\right)\right.
$$$$
\left.+\frac{b_{12}^{* 1}}{b_{11}^{* 1}} \varphi_{2}^{*}\left(r-m_{n}\right)\right]
$$

$$
\begin{aligned}
& +b_{11}^{* n-1}\left(\sum _ { r = 0 } ^ { m _ { n - 1 } } \lambda ^ { k - 1 - r } \left[\varphi_{1}^{*}\left(r-m_{n-1}\right)\right.\right. \\
& \left.+\frac{b_{12}^{* 1}}{b_{11}^{* 1}} \varphi_{2}^{*}\left(r-m_{n-1}\right)\right] \\
& +\left(m_{n}-m_{n-1}\right) \lambda^{k-1-m_{n-1}} \\
& \left.\times\left[\varphi_{1}^{*}(0)+\frac{b_{12}^{* 1}}{b_{11}^{* 1}} \varphi_{2}^{*}(0)\right]\right) \\
& +\left(k-1-m_{n}\right) \\
& \times\left(\lambda^{k-1-m_{1}} b_{11}^{* 1}\left[\varphi_{1}^{*}(0)+\frac{b_{12}^{* 1}}{b_{11}^{* 1}} \varphi_{2}^{*}(0)\right]\right. \\
& +\lambda^{k-1-m_{2}} b_{11}^{* 2}\left[\varphi_{1}^{*}(0)+\frac{b_{12}^{* 1}}{b_{11}^{* 1}} \varphi_{2}^{*}(0)\right]+\cdots \\
& +\lambda^{k-1-m_{n-1}} b_{11}^{* n-1}\left[\varphi_{1}^{*}(0)+\frac{b_{12}^{* 1}}{b_{11}^{* 1}} \varphi_{2}^{*}(0)\right] \\
& \left.+\lambda^{k-1-m_{n}} b_{11}^{* n}\left[\varphi_{1}^{*}(0)+\frac{b_{12}^{* 1}}{b_{11}^{* 1}} \varphi_{2}^{*}(0)\right]\right)
\end{aligned}
$$$$
\left.\times\left[\varphi_{1}^{*}(0)+\frac{b_{12}^{* 1}}{b_{11}^{* 1}} \varphi_{2}^{*}(0)\right]\right)
$$ 


$$
\begin{aligned}
& =\lambda^{k} \varphi_{1}^{*}(0)+b_{11}^{* 1}\left(\sum _ { r = 0 } ^ { m _ { 1 } } \lambda ^ { k - 1 - r } \left[\varphi_{1}^{*}\left(r-m_{1}\right)\right.\right. \\
& \left.+\frac{b_{12}^{* 1}}{b_{11}^{* 1}} \varphi_{2}^{*}\left(r-m_{1}\right)\right] \\
& +\left(k-1-m_{1}\right) \lambda^{k-1-m_{1}} \\
& \left.\times\left[\varphi_{1}^{*}(0)+\frac{b_{12}^{* 1}}{b_{11}^{* 1}} \varphi_{2}^{*}(0)\right]\right) \\
& +b_{11}^{* 2}\left(\sum_{r=0}^{m_{2}} \lambda^{k-1-r}\left[\varphi_{1}^{*}\left(r-m_{2}\right)+\frac{b_{12}^{* 1}}{b_{11}^{* 1}} \varphi_{2}^{*}\left(r-m_{2}\right)\right]\right. \\
& +\left(k-1-m_{2}\right) \lambda^{k-1-m_{2}} \\
& \left.\times\left[\varphi_{1}^{*}(0)+\frac{b_{12}^{* 1}}{b_{11}^{* 1}} \varphi_{2}^{*}(0)\right]\right) \\
& +\cdots \\
& +b_{11}^{* n-1}\left(\sum _ { r = 0 } ^ { m _ { n - 1 } } \lambda ^ { k - 1 - r } \left[\varphi_{1}^{*}\left(r-m_{n-1}\right)\right.\right. \\
& \left.+\frac{b_{12}^{* 1}}{b_{11}^{* 1}} \varphi_{2}^{*}\left(r-m_{n-1}\right)\right] \\
& +\left(k-1-m_{n-1}\right) \lambda^{k-1-m_{n-1}} \\
& \left.\times\left[\varphi_{1}^{*}(0)+\frac{b_{12}^{* 1}}{b_{11}^{* 1}} \varphi_{2}^{*}(0)\right]\right) \\
& +b_{11}^{* n}\left(\sum_{r=0}^{m_{n}} \lambda^{k-1-r}\left[\varphi_{1}^{*}\left(r-m_{n}\right)+\frac{b_{12}^{* 1}}{b_{11}^{* 1}} \varphi_{2}^{*}\left(r-m_{n}\right)\right]\right. \\
& +\left(k-1-m_{n}\right) \lambda^{k-1-m_{n}} \\
& \left.\times\left[\varphi_{1}^{*}(0)+\frac{b_{12}^{* 1}}{b_{11}^{* 1}} \varphi_{2}^{*}(0)\right]\right) \\
& =\lambda^{k} \varphi_{1}^{*}(0) \\
& +\sum_{l=1}^{n} b_{11}^{* l}\left(\sum_{r=0}^{m_{l}} \lambda^{k-1-r}\left[\varphi_{1}^{*}\left(r-m_{l}\right)+\frac{b_{12}^{* 1}}{b_{11}^{* 1}} \varphi_{2}^{*}\left(r-m_{l}\right)\right]\right. \\
& +\left(k-1-m_{l}\right) \lambda^{k-1-m_{l}} \\
& \left.\times\left[\varphi_{1}^{*}(0)+\frac{b_{12}^{* 1}}{b_{11}^{* 1}} \varphi_{2}^{*}(0)\right]\right), \\
& =\lambda^{k-\left(m_{n}+1\right)} y_{2}\left(m_{n}+1\right)-\sum_{r=m_{n}+1}^{k-1} \lambda^{k-1-r} \\
& \times\left(\sum_{l=1}^{n} \frac{\left(b_{11}^{* l}\right)^{2}}{b_{12}^{* l}} \lambda^{r-m_{l}}\left[\varphi_{1}^{*}(0)+\frac{b_{12}^{* 1}}{b_{11}^{* 1}} \varphi_{2}^{*}(0)\right]\right) \\
& =\lambda^{k-m_{n}-1} \\
& \times\left[\lambda^{m_{n}+1} \varphi_{2}^{*}(0)-\sum_{r=0}^{m_{n}} \lambda^{m_{n}-r} \frac{\left(b_{11}^{* n}\right)^{2}}{b_{12}^{* n}}\right. \\
& \times\left[\varphi_{1}^{*}\left(r-m_{n}\right)+\frac{b_{12}^{* 1}}{b_{11}^{* 1}} \varphi_{2}^{*}\left(r-m_{n}\right)\right] \\
& -\sum_{l=1}^{n-1} \frac{\left(b_{11}^{* l}\right)^{2}}{b_{12}^{* l}} \\
& \times\left(\sum _ { r = 0 } ^ { m _ { l } } \lambda ^ { m _ { n } - r } \left[\varphi_{1}^{*}\left(r-m_{l}\right)\right.\right. \\
& \left.+\frac{b_{12}^{* 1}}{b_{11}^{* 1}} \varphi_{2}^{*}\left(r-m_{l}\right)\right] \\
& +\left(m_{n}-m_{l}\right) \lambda^{m_{n}-m_{l}} \\
& \left.\left.\times\left[\varphi_{1}^{*}(0)+\frac{b_{12}^{* 1}}{b_{11}^{* 1}} \varphi_{2}^{*}(0)\right]\right)\right] \\
& -\sum_{r=m_{n}+1}^{k-1} \lambda^{k-1-r}\left(\sum_{l=1}^{n} \frac{\left(b_{11}^{* l}\right)^{2}}{b_{12}^{* l}} \lambda^{r-m_{l}}\right. \\
& \left.\times\left[\varphi_{1}^{*}(0)+\frac{b_{12}^{* 1}}{b_{11}^{* 1}} \varphi_{2}^{*}(0)\right]\right) \\
& =\lambda^{k} \varphi_{2}^{*}(0)-\sum_{r=0}^{m_{n}} \lambda^{k-1-r} \frac{\left(b_{11}^{* n}\right)^{2}}{b_{12}^{* n}} \\
& \times\left[\varphi_{1}^{*}\left(r-m_{n}\right)+\frac{b_{12}^{* 1}}{b_{11}^{* 1}} \varphi_{2}^{*}\left(r-m_{n}\right)\right] \\
& -\sum_{l=1}^{n-1} \frac{\left(b_{11}^{* l}\right)^{2}}{b_{12}^{* l}}\left(\sum _ { r = 0 } ^ { m _ { l } } \lambda ^ { k - 1 - r } \left[\varphi_{1}^{*}\left(r-m_{l}\right)\right.\right. \\
& \left.+\frac{b_{12}^{* 1}}{b_{11}^{* 1}} \varphi_{2}^{*}\left(r-m_{l}\right)\right] \\
& +\left(m_{n}-m_{l}\right) \lambda^{k-1-m_{l}} \\
& \left.\times\left[\varphi_{1}^{*}(0)+\frac{b_{12}^{* 1}}{b_{11}^{* 1}} \varphi_{2}^{*}(0)\right]\right)
\end{aligned}
$$


26

Abstract and Applied Analysis

$$
\begin{aligned}
& -\sum_{r=m_{n}+1}^{k-1} \lambda^{k-1-r}\left(\sum_{l=1}^{n} \frac{\left(b_{11}^{* l}\right)^{2}}{b_{12}^{* l}} \lambda^{r-m_{l}}\right. \\
& +\lambda^{k-1-m_{n-1}} \frac{\left(b_{11}^{* n-1}\right)^{2}}{b_{12}^{* n-1}}\left[\varphi_{1}^{*}(0)+\frac{b_{12}^{* 1}}{b_{11}^{* 1}} \varphi_{2}^{*}(0)\right] \\
& \left.\times\left[\varphi_{1}^{*}(0)+\frac{b_{12}^{* 1}}{b_{11}^{* 1}} \varphi_{2}^{*}(0)\right]\right) \\
& \left.+\lambda^{k-1-m_{n}} \frac{\left(b_{11}^{* n}\right)^{2}}{b_{12}^{* n}}\left[\varphi_{1}^{*}(0)+\frac{b_{12}^{* 1}}{b_{11}^{* 1}} \varphi_{2}^{*}(0)\right]\right) \\
& =\lambda^{k} \varphi_{2}^{*}(0)-\sum_{r=0}^{m_{n}} \lambda^{k-1-r} \frac{\left(b_{11}^{* n}\right)^{2}}{b_{12}^{* n}}\left[\varphi_{1}^{*}\left(r-m_{n}\right)\right. \\
& \left.+\frac{b_{12}^{* 1}}{b_{11}^{* 1}} \varphi_{2}^{*}\left(r-m_{n}\right)\right] \\
& =\lambda^{k} \varphi_{2}^{*}(0)-\frac{\left(b_{11}^{* 1}\right)^{2}}{b_{12}^{* 1}} \\
& \times\left(\sum_{r=0}^{m_{1}} \lambda^{k-1-r}\left[\varphi_{1}^{*}\left(r-m_{1}\right)+\frac{b_{12}^{* 1}}{b_{11}^{* 1}} \varphi_{2}^{*}\left(r-m_{1}\right)\right]\right. \\
& -\frac{\left(b_{11}^{* 1}\right)^{2}}{b_{12}^{* 1}}\left(\sum _ { r = 0 } ^ { m _ { 1 } } \lambda ^ { k - 1 - r } \left[\varphi_{1}^{*}\left(r-m_{1}\right)\right.\right. \\
& +\left(k-1-m_{1}\right) \lambda^{k-1-m_{1}} \\
& \left.+\frac{b_{12}^{* 1}}{b_{11}^{* 1}} \varphi_{2}^{*}\left(r-m_{1}\right)\right] \\
& +\left(m_{n}-m_{1}\right) \lambda^{k-1-m_{1}} \\
& \left.\times\left[\varphi_{1}^{*}(0)+\frac{b_{12}^{* 1}}{b_{11}^{* 1}} \varphi_{2}^{*}(0)\right]\right) \\
& \left.\times\left[\varphi_{1}^{*}(0)+\frac{b_{12}^{* 1}}{b_{11}^{* 1}} \varphi_{2}^{*}(0)\right]\right) \\
& -\frac{\left(b_{11}^{* 2}\right)^{2}}{b_{12}^{* 2}}\left(\sum _ { r = 0 } ^ { m _ { 2 } } \lambda ^ { k - 1 - r } \left[\varphi_{1}^{*}\left(r-m_{2}\right)\right.\right. \\
& -\frac{\left(b_{11}^{* 2}\right)^{2}}{b_{12}^{* 2}}\left(\sum _ { r = 0 } ^ { m _ { 2 } } \lambda ^ { k - 1 - r } \left[\varphi_{1}^{*}\left(r-m_{2}\right)\right.\right. \\
& \left.+\frac{b_{12}^{* 1}}{b_{11}^{* 1}} \varphi_{2}^{*}\left(r-m_{2}\right)\right] \\
& \left.+\frac{b_{12}^{* 1}}{b_{11}^{* 1}} \varphi_{2}^{*}\left(r-m_{2}\right)\right] \\
& +\left(m_{n}-m_{2}\right) \lambda^{k-1-m_{2}} \\
& \left.\times\left[\varphi_{1}^{*}(0)+\frac{b_{12}^{* 1}}{b_{11}^{* 1}} \varphi_{2}^{*}(0)\right]\right) \\
& +\cdots \\
& -\frac{\left(b_{11}^{* n-1}\right)^{2}}{b_{12}^{* n-1}}\left(\sum _ { r = 0 } ^ { m _ { n - 1 } } \lambda ^ { k - 1 - r } \left[\varphi_{1}^{*}\left(r-m_{n-1}\right)\right.\right. \\
& \left.+\frac{b_{12}^{* 1}}{b_{11}^{* 1}} \varphi_{2}^{*}\left(r-m_{n-1}\right)\right] \\
& +\left(m_{n}-m_{n-1}\right) \lambda^{k-1-m_{n-1}} \\
& \left.\times\left[\varphi_{1}^{*}(0)+\frac{b_{12}^{* 1}}{b_{11}^{* 1}} \varphi_{2}^{*}(0)\right]\right)-\left(k-1-m_{n}\right) \\
& \times\left(\lambda^{k-1-m_{1}} \frac{\left(b_{11}^{* 1}\right)^{2}}{b_{12}^{* 1}}\left[\varphi_{1}^{*}(0)+\frac{b_{12}^{* 1}}{b_{11}^{* 1}} \varphi_{2}^{*}(0)\right]\right. \\
& +\left(k-1-m_{2}\right) \lambda^{k-1-m_{2}} \\
& \left.\times\left[\varphi_{1}^{*}(0)+\frac{b_{12}^{* 1}}{b_{11}^{* 1}} \varphi_{2}^{*}(0)\right]\right) \\
& +\cdots \\
& -\frac{\left(b_{11}^{* n-1}\right)^{2}}{b_{12}^{* n-1}}\left(\sum _ { r = 0 } ^ { m _ { n - 1 } } \lambda ^ { k - 1 - r } \left[\varphi_{1}^{*}\left(r-m_{n-1}\right)\right.\right. \\
& \left.+\frac{b_{12}^{* 1}}{b_{11}^{* 1}} \varphi_{2}^{*}\left(r-m_{n-1}\right)\right] \\
& +\left(k-1-m_{n-1}\right) \lambda^{k-1-m_{n-1}} \\
& \left.\times\left[\varphi_{1}^{*}(0)+\frac{b_{12}^{* 1}}{b_{11}^{* 1}} \varphi_{2}^{*}(0)\right]\right) \\
& +\lambda^{k-1-m_{2}} \frac{\left(b_{11}^{* 2}\right)^{2}}{b_{12}^{* 2}}\left[\varphi_{1}^{*}(0)+\frac{b_{12}^{* 1}}{b_{11}^{* 1}} \varphi_{2}^{*}(0)\right]+\cdots \\
& -\frac{\left(b_{11}^{* n}\right)^{2}}{b_{12}^{* n}}\left(\sum _ { r = 0 } ^ { m _ { n } } \lambda ^ { k - 1 - r } \left[\varphi_{1}^{*}\left(r-m_{n}\right)\right.\right.
\end{aligned}
$$


Abstract and Applied Analysis

27

$$
\begin{array}{r}
\left.+\frac{b_{12}^{* 1}}{b_{11}^{* 1}} \varphi_{2}^{*}\left(r-m_{n}\right)\right] \\
+\left(k-1-m_{n}\right) \lambda^{k-1-m_{n}} \\
\left.\times\left[\varphi_{1}^{*}(0)+\frac{b_{12}^{* 1}}{b_{11}^{* 1}} \varphi_{2}^{*}(0)\right]\right) \\
=\lambda^{k} \varphi_{2}^{*}(0)-\sum_{l=1}^{n} \frac{\left(b_{11}^{* l}\right)^{2}}{b_{12}^{* l}}
\end{array}
$$

$$
\begin{aligned}
\times & \left(\sum_{r=0}^{m_{l}} \lambda^{k-1-r}\left[\varphi_{1}^{*}\left(r-m_{l}\right)+\frac{b_{12}^{* 1}}{b_{11}^{* 1}} \varphi_{2}^{*}\left(r-m_{l}\right)\right]\right. \\
& +\left(k-1-m_{l}\right) \lambda^{k-1-m_{l}} \\
& \left.\times\left[\varphi_{1}^{*}(0)+\frac{b_{12}^{* 1}}{b_{11}^{* 1}} \varphi_{2}^{*}(0)\right]\right) .
\end{aligned}
$$

Summing up all particular cases (90), (93), (96), (100), and (103) we have

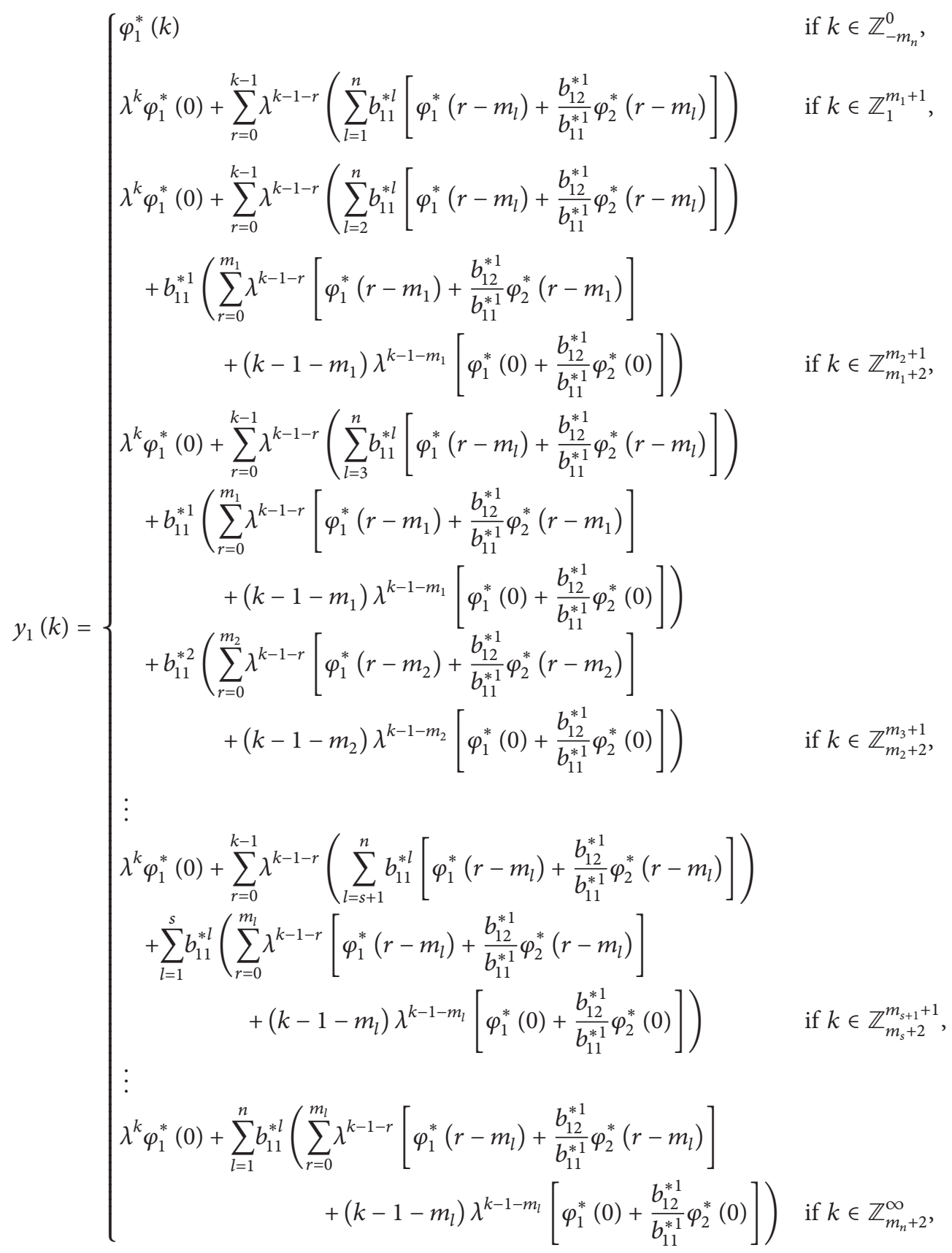


and from cases (91), (94), (97), (101), and (104) we conclude that

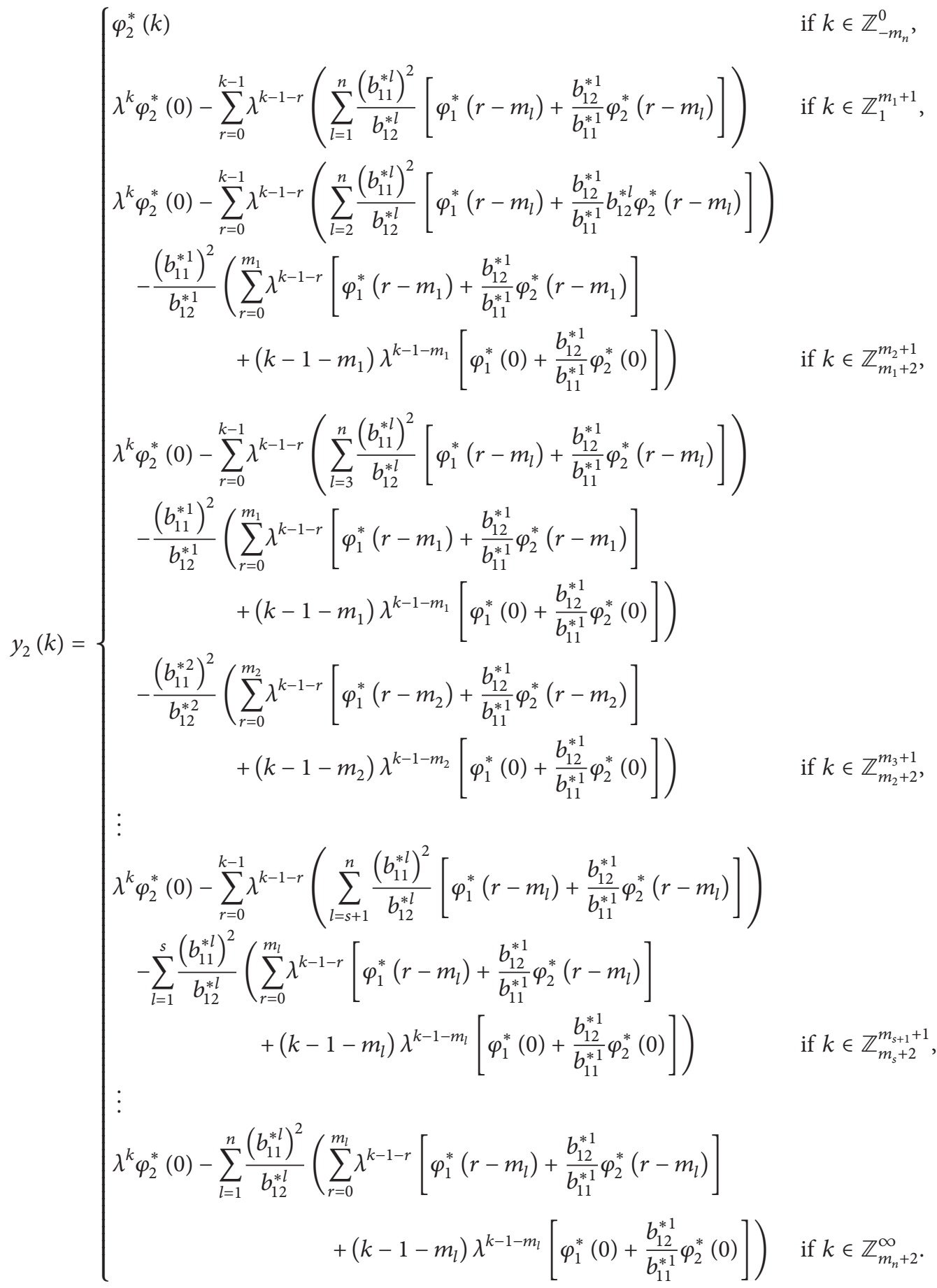

Formula (81) is now a direct consequence of (105), (106), and (80).

2.1.6. Case (39) of a Double Real Root. If the matrix $\Lambda$ has the form (39), the necessary and sufficient conditions (13)-(16), for (40), are reduced to (43), (44), (46), and

$$
\left|\begin{array}{cc}
\lambda & 1 \\
b_{21}^{* l} & b_{22}^{* l}
\end{array}\right|+\left|\begin{array}{cc}
b_{11}^{* l} & b_{12}^{* l} \\
0 & \lambda
\end{array}\right|=\lambda\left(b_{11}^{* l}+b_{22}^{* l}\right)-b_{21}^{* l}=0
$$

Then (43), (44), and (107) give $b_{11}^{* l}=b_{22}^{* l}=b_{21}^{* l}=0$.

Theorem 9. Let (1) be a weakly delayed system, (35) has a double root $\lambda_{1,2}=\lambda$ and the matrix $\Lambda$ has the form (39). Then 
$b_{11}^{* l}=b_{22}^{* l}=b_{21}^{* l}=0$ and the solution of the initial problem (1), (3) is $x(k)=\delta y(k), y(k)=\left(y_{1}(k), y_{2}(k)\right)^{T}$, and

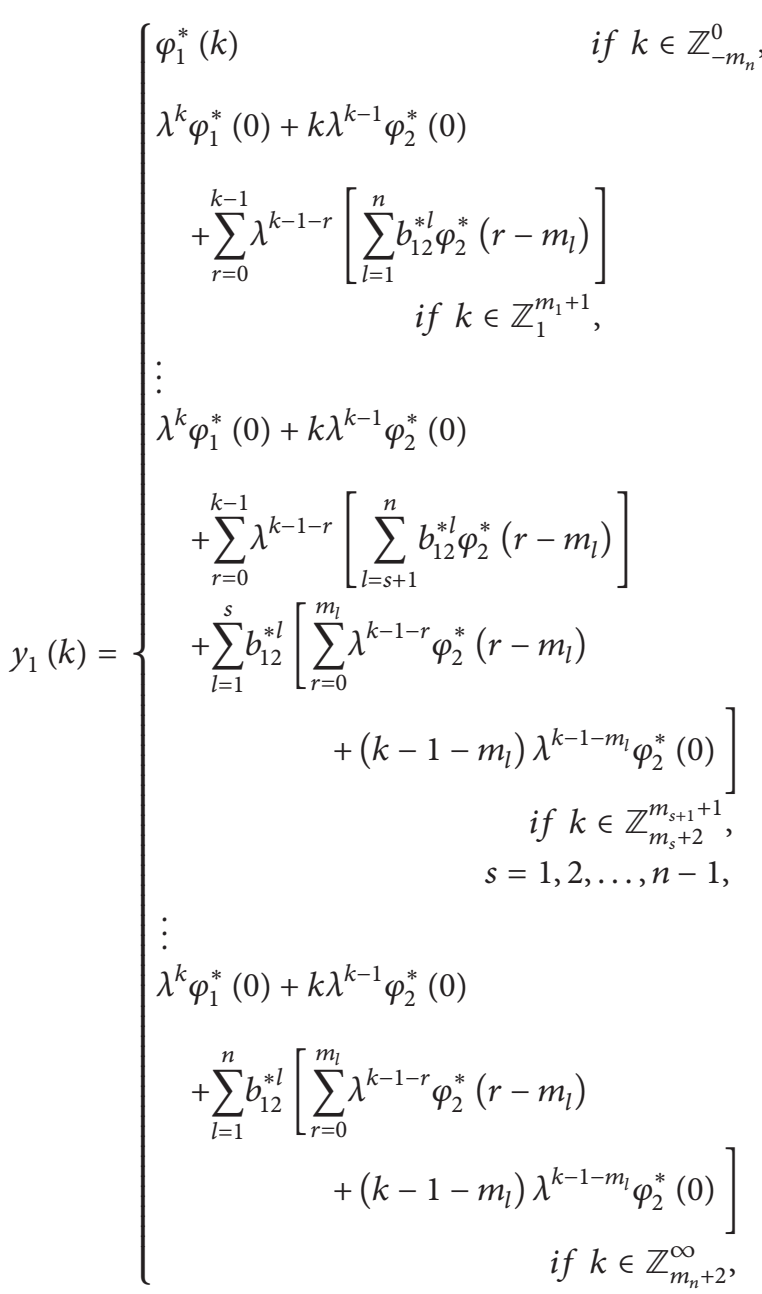

$$
\begin{aligned}
& y_{2}(k)= \begin{cases}\varphi_{2}^{*}(k) & \text { if } k \in \mathbb{Z}_{-m_{n}}^{0}, \\
\lambda^{k} \varphi_{2}^{*}(0) & \text { if } k \in \mathbb{Z}_{1}^{\infty} .\end{cases}
\end{aligned}
$$

Proof. The system (40) can be written as

$$
\begin{gathered}
y_{1}(k+1)=\lambda y_{1}(k)+y_{2}(k)+\sum_{l=1}^{n} b_{12}^{* l} y_{2}\left(k-m_{l}\right), \\
y_{2}(k+1)=\lambda y_{2}(k), \quad k \in \mathbb{Z}_{0}^{\infty} .
\end{gathered}
$$

Solving (111), we get

$$
y_{2}(k)= \begin{cases}\varphi_{2}^{*}(k) & \text { if } k \in \mathbb{Z}_{-m_{n}}^{0}, \\ \lambda^{k} \varphi_{2}^{*}(0) & \text { if } k \in \mathbb{Z}_{1}^{\infty},\end{cases}
$$

then (110) turns into

$$
y_{1}(k+1)=\left\{\begin{array}{c}
\lambda y_{1}(k)+\lambda^{k} \varphi_{2}^{*}(0)+\sum_{l=1}^{n} b_{12}^{* l} \varphi_{2}^{*}\left(k-m_{l}\right) \\
\text { if } k \in \mathbb{Z}_{0}^{m_{1}}, \\
\lambda y_{1}(k)+\lambda^{k} \varphi_{2}^{*}(0)+b_{12}^{* 1} \lambda^{k-m_{1}} \varphi_{2}^{*}(0) \\
+\sum_{l=2}^{n} b_{12}^{* l} \varphi_{2}^{*}\left(k-m_{l}\right) \\
\text { if } k \in \mathbb{Z}_{m_{1}+1}^{m_{2}}, \\
\lambda y_{1}(k)+\lambda^{k} \varphi_{2}^{*}(0)+\sum_{l=1}^{2} b_{12}^{* l} \lambda^{k-m_{l}} \varphi_{2}^{*}(0) \\
+\sum_{l=3}^{n} b_{12}^{* l} \varphi_{2}^{*}\left(k-m_{l}\right) \\
\quad \text { if } k \in \mathbb{Z}_{m_{2}+1}^{m_{3}}, \\
\lambda y_{1}(k)+\lambda^{k} \varphi_{2}^{*}(0)+\sum_{l=1}^{s} b_{12}^{* l} \lambda^{k-m_{l}} \varphi_{2}^{*}(0) \\
+\sum_{l=s+1}^{n} b_{12}^{* l} \varphi_{2}^{*}\left(k-m_{l}\right) \\
\text { if } k \in \mathbb{Z}_{m_{s}+1}^{m_{s+1}}, \\
s=3,4, \ldots, n-1, \\
\vdots \begin{array}{l}
\lambda y_{1}(k)+\lambda^{k} \varphi_{2}^{*}(0)+\sum_{l=1}^{n} b_{12}^{* l} \lambda^{k-m_{l}} \varphi_{2}^{*}(0) \\
\text { if } k \in \mathbb{Z}_{m_{n}+1}^{\infty} .
\end{array}
\end{array}\right.
$$

Equation (113) can be solved in a way similar to that of (54) in the proof of Theorem 5 using (33).

First we solve (113) for $k \in \mathbb{Z}_{0}^{m_{1}}$. This means that we consider the problem

$$
\begin{aligned}
y_{1}(k+1)= & \lambda y_{1}(k)+\lambda^{k} \varphi_{2}^{*}(0) \\
& +\sum_{l=1}^{n} b_{12}^{* l} \varphi_{2}^{*}\left(k-m_{l}\right) \quad \text { if } k \in \mathbb{Z}_{0}^{m_{1}}, \\
& y_{1}(0)=\varphi_{1}^{*}(0) .
\end{aligned}
$$

With the aid of formula (33), we get

$$
\begin{aligned}
y_{1}(k)= & \lambda^{k} \varphi_{1}^{*}(0) \\
& +\sum_{r=0}^{k-1} \lambda^{k-1-r}\left[\lambda^{r} \varphi_{2}^{*}(0)+\sum_{l=1}^{n} b_{12}^{* l} \varphi_{2}^{*}\left(r-m_{l}\right)\right]
\end{aligned}
$$




$$
\begin{aligned}
= & \lambda^{k} \varphi_{1}^{*}(0)+k \lambda^{k-1} \varphi_{2}^{*}(0) \\
& +\sum_{r=0}^{k-1} \lambda^{k-1-r}\left[\sum_{l=1}^{n} b_{12}^{* l} \varphi_{2}^{*}\left(r-m_{l}\right)\right], \quad k \in \mathbb{Z}_{1}^{m_{1}+1} .
\end{aligned}
$$

Now we solve (113) for $k \in \mathbb{Z}_{m_{1}+1}^{m_{2}}$ with initial data deduced from (115); that is, we consider the problem

$$
\begin{aligned}
y_{1}(k+1)= & \lambda y_{1}(k)+\lambda^{k} \varphi_{2}^{*}(0)+b_{12}^{* 1} \lambda^{k-m_{1}} \varphi_{2}^{*}(0) \\
& +\sum_{l=2}^{n} b_{12}^{* l} \varphi_{2}^{*}\left(k-m_{l}\right), \quad k \in \mathbb{Z}_{m_{1}+1}^{m_{2}}, \\
y_{1}\left(m_{1}+1\right)= & \lambda^{m_{1}+1} \varphi_{1}^{*}(0)+\left(m_{1}+1\right) \lambda^{m_{1}} \varphi_{2}^{*}(0) \\
& +\sum_{r=0}^{m_{1}} \lambda^{m_{1}-r}\left[\sum_{l=1}^{n} b_{12}^{* l} \varphi_{2}^{*}\left(r-m_{l}\right)\right] .
\end{aligned}
$$

Applying formula (33) we get (for $k \in \mathbb{Z}_{m_{1}+2}^{m_{2}+1}$ )

$$
\begin{aligned}
& y_{1}(k)=\lambda^{k-\left(m_{1}+1\right)} y_{1}\left(m_{1}+1\right) \\
& +\sum_{r=m_{1}+1}^{k-1} \lambda^{k-1-r}\left[\lambda^{r} \varphi_{2}^{*}(0)+b_{12}^{* 1} \lambda^{r-m_{1}} \varphi_{2}^{*}(0)\right. \\
& \left.+\sum_{l=2}^{n} b_{12}^{* l} \varphi_{2}^{*}\left(r-m_{l}\right)\right] \\
& =\lambda^{k-m_{1}-1}\left[\lambda^{m_{1}+1} \varphi_{1}^{*}(0)+\left(m_{1}+1\right) \lambda^{m_{1}} \varphi_{2}^{*}(0)\right. \\
& \left.+\sum_{r=0}^{m_{1}} \lambda^{m_{1}-r}\left[\sum_{l=1}^{n} b_{12}^{* l} \varphi_{2}^{*}\left(r-m_{l}\right)\right]\right] \\
& +\sum_{r=m_{1}+1}^{k-1} \lambda^{k-1-r}\left[\lambda^{r} \varphi_{2}^{*}(0)+b_{12}^{* 1} \lambda^{r-m_{1}} \varphi_{2}^{*}(0)\right. \\
& \left.+\sum_{l=2}^{n} b_{12}^{* l} \varphi_{2}^{*}\left(r-m_{l}\right)\right] \\
& =\lambda^{k} \varphi_{1}^{*}(0)+\left(m_{1}+1\right) \lambda^{k-1} \varphi_{2}^{*}(0) \\
& +\sum_{r=0}^{m_{1}} \lambda^{k-1-r}\left[\sum_{l=1}^{n} b_{12}^{* l} \varphi_{2}^{*}\left(r-m_{l}\right)\right]
\end{aligned}
$$

$$
\begin{aligned}
& +\left(k-m_{1}-1\right) \lambda^{k-1} \varphi_{2}^{*}(0) \\
& +\sum_{r=m_{1}+1}^{k-1} \lambda^{k-1-r}\left[b_{12}^{* 1} \lambda^{r-m_{1}} \varphi_{2}^{*}(0)+\sum_{l=2}^{n} b_{12}^{* l} \varphi_{2}^{*}\left(r-m_{l}\right)\right] \\
& =\lambda^{k} \varphi_{1}^{*}(0)+k \lambda^{k-1} \varphi_{2}^{*}(0) \\
& +\sum_{r=0}^{k-1} \lambda^{k-1-r}\left[\sum_{l=2}^{n} b_{12}^{* l} \varphi_{2}^{*}\left(r-m_{l}\right)\right] \\
& +b_{12}^{* 1}\left[\sum_{r=0}^{m_{1}} \lambda^{k-1-r} \varphi_{2}^{*}\left(r-m_{1}\right)\right. \\
& \left.+\left(k-1-m_{1}\right) \lambda^{k-1-m_{1}} \varphi_{2}^{*}(0)\right] .
\end{aligned}
$$

Now we solve (113) for $k \in \mathbb{Z}_{m_{2}+1}^{m_{3}}$ with initial data deduced from (117); that is, we consider the problem

$$
\begin{aligned}
& y_{1}(k+1)=\lambda y_{1}(k)+\lambda^{k} \varphi_{2}^{*}(0)+\sum_{l=1}^{2} b_{12}^{* l} \lambda^{k-m_{l}} \varphi_{2}^{*}(0) \\
& +\sum_{l=3}^{n} b_{12}^{* l} \varphi_{2}^{*}\left(k-m_{l}\right) \quad k \in \mathbb{Z}_{m_{2}+1}^{m_{3}} \\
& y_{1}\left(m_{2}+1\right)=\lambda^{m_{2}+1} \varphi_{1}^{*}(0)+\left(m_{2}+1\right) \lambda^{m_{2}} \varphi_{2}^{*}(0) \\
& +\sum_{r=0}^{m_{2}} \lambda^{m_{2}-r}\left[\sum_{l=2}^{n} b_{12}^{* l} \varphi_{2}^{*}\left(r-m_{l}\right)\right] \\
& +b_{12}^{* 1}\left[\sum_{r=0}^{m_{1}} \lambda^{m_{2}-r} \varphi_{2}^{*}\left(r-m_{1}\right)\right. \\
& \left.+\left(m_{2}-m_{1}\right) \lambda^{m_{2}-m_{1}} \varphi_{2}^{*}(0)\right] . \\
& y_{1}(k)=\lambda^{k-\left(m_{2}+1\right)} y_{1}\left(m_{2}+1\right) \\
& +\sum_{r=m_{2}+1}^{k-1} \lambda^{k-1-r}\left[\lambda^{r} \varphi_{2}^{*}(0)+\sum_{l=1}^{2} b_{12}^{* l} \lambda^{r-m_{l}} \varphi_{2}^{*}(0)\right. \\
& \left.+\sum_{l=3}^{n} b_{12}^{* l} \varphi_{2}^{*}\left(r-m_{l}\right)\right]
\end{aligned}
$$




$$
\begin{aligned}
& =\lambda^{k-m_{2}-1}\left[\lambda^{m_{2}+1} \varphi_{1}^{*}(0)+\left(m_{2}+1\right) \lambda^{m_{2}} \varphi_{2}^{*}(0)\right. \\
& +\sum_{r=0}^{m_{2}} \lambda^{m_{2}-r}\left[\sum_{l=2}^{n} b_{12}^{* l} \varphi_{2}^{*}\left(r-m_{l}\right)\right] \\
& +b_{12}^{* 1}\left[\sum_{r=0}^{m_{1}} \lambda^{m_{2}-r} \varphi_{2}^{*}\left(r-m_{1}\right)\right. \\
& \left.\left.+\left(m_{2}-m_{1}\right) \lambda^{m_{2}-m_{1}} \varphi_{2}^{*}(0)\right]\right] \\
& +\sum_{r=m_{2}+1}^{k-1} \lambda^{k-1-r}\left[\lambda^{r} \varphi_{2}^{*}(0)+\sum_{l=1}^{2} b_{12}^{* l} \lambda^{r-m_{l}} \varphi_{2}^{*}(0)\right. \\
& \left.+\sum_{l=3}^{n} b_{12}^{* l} \varphi_{2}^{*}\left(r-m_{l}\right)\right] \\
& =\lambda^{k} \varphi_{1}^{*}(0)+\left(m_{2}+1\right) \lambda^{k-1} \varphi_{2}^{*}(0) \\
& +\sum_{r=0}^{m_{2}} \lambda^{k-1-r}\left[\sum_{l=2}^{n} b_{12}^{* l} \varphi_{2}^{*}\left(r-m_{l}\right)\right] \\
& +b_{12}^{* 1}\left[\sum_{r=0}^{m_{1}} \lambda^{k-1-r} \varphi_{2}^{*}\left(r-m_{1}\right)\right. \\
& \left.+\left(m_{2}-m_{1}\right) \lambda^{k-1-m_{1}} \varphi_{2}^{*}(0)\right] \\
& +\left(k-1-m_{2}\right) \lambda^{k-1} \varphi_{2}^{*}(0) \\
& +\sum_{r=m_{2}+1}^{k-1} \lambda^{k-1-r}\left[\sum_{l=1}^{2} b_{12}^{* l} \lambda^{r-m_{l}} \varphi_{2}^{*}(0)\right. \\
& \left.+\sum_{l=3}^{n} b_{12}^{* l} \varphi_{2}^{*}\left(r-m_{l}\right)\right] \\
& =\lambda^{k} \varphi_{1}^{*}(0)+k \lambda^{k-1} \varphi_{2}^{*}(0) \\
& +\sum_{r=0}^{k-1} \lambda^{k-1-r}\left[\sum_{l=3}^{n} b_{12}^{* l} \varphi_{2}^{*}\left(r-m_{l}\right)\right] \\
& +b_{12}^{* 1}\left[\sum_{r=0}^{m_{1}} \lambda^{k-1-r} \varphi_{2}^{*}\left(r-m_{1}\right)\right. \\
& \left.+\left(k-1-m_{1}\right) \lambda^{k-1-m_{1}} \varphi_{2}^{*}(0)\right]
\end{aligned}
$$

From (115), (117), and (119) we deduce that expected form of the solution of the initial problem for $k \in \mathbb{Z}_{m_{s-1}+1}^{m_{s}}$ with initial data derived from the solution of previous equation for $k \in \mathbb{Z}_{m_{s-2}+1}^{m_{s-1}}$ is

$$
\begin{aligned}
& y_{1}(k)= \lambda^{k} \varphi_{1}^{*}(0)+k \lambda^{k-1} \varphi_{2}^{*}(0) \\
&+\sum_{r=0}^{k-1} \lambda^{k-1-r}\left[\sum_{l=s}^{n} b_{12}^{* l} \varphi_{2}^{*}\left(r-m_{l}\right)\right] \\
&+\sum_{l=1}^{s-1} b_{12}^{* l}\left[\sum_{r=0}^{m_{l}} \lambda^{k-1-r} \varphi_{2}^{*}\left(r-m_{l}\right)\right. \\
&\left.+\left(k-1-m_{l}\right) \lambda^{k-1-m_{l}} \varphi_{2}^{*}(0)\right] \\
& \text { if } k \in \mathbb{Z}_{m_{s-1}+2}^{m_{s}+1} .
\end{aligned}
$$

We solve (113) for $k \in \mathbb{Z}_{m_{s}+1}^{m_{s+1}}$ with initial data deduced from (120); that is, we consider the problem

$$
\begin{aligned}
y_{1}(k+1)= & \lambda y_{1}(k)+\lambda^{k} \varphi_{2}^{*}(0)+\sum_{l=1}^{s} b_{12}^{* l} \lambda^{k-m_{l}} \varphi_{2}^{*}(0) \\
& +\sum_{l=s+1}^{n} b_{12}^{* l} \varphi_{2}^{*}\left(k-m_{l}\right), \quad k \in \mathbb{Z}_{m_{s}+1}^{m_{s+1}}, \\
y_{1}\left(m_{s}+1\right)= & \lambda^{m_{s}+1} \varphi_{1}^{*}(0)+\left(m_{s}+1\right) \lambda^{m_{s}} \varphi_{2}^{*}(0) \\
& +\sum_{r=0}^{m_{s}} \lambda^{m_{s}-r}\left[\sum_{l=s}^{n} b_{12}^{* l} \varphi_{2}^{*}\left(r-m_{l}\right)\right] \\
& +\sum_{l=1}^{s-1} b_{12}^{* l}\left[\sum_{r=0}^{m_{l}} \lambda^{m_{s}-r} \varphi_{2}^{*}\left(r-m_{l}\right)\right. \\
& \left.+\left(m_{s}-m_{l}\right) \lambda^{m_{s}-m_{l}} \varphi_{2}^{*}(0)\right] .
\end{aligned}
$$


Applying formula (33) yields (for $k \in \mathbb{Z}_{m_{s}+2}^{m_{s+1}+1}$ )

$$
+\sum_{r=0}^{m_{s}} \lambda^{k-1-r} b_{12}^{* s} \varphi_{2}^{*}\left(r-m_{s}\right)
$$

$$
\begin{aligned}
y_{1}(k)= & \lambda^{k-\left(m_{s}+1\right)} y_{1}\left(m_{s}+1\right) \\
+ & \sum_{r=m_{s}+1}^{k-1} \lambda^{k-1-r}\left[\lambda^{r} \varphi_{2}^{*}(0)+\sum_{l=1}^{s} b_{12}^{* l} \lambda^{r-m_{l}} \varphi_{2}^{*}(0)\right. \\
& \left.+\sum_{l=s+1}^{n} b_{12}^{* l} \varphi_{2}^{*}\left(r-m_{l}\right)\right]
\end{aligned}
$$$$
=\lambda^{k-m_{s}-1}
$$$$
+b_{12}^{* 1}\left[\sum_{r=0}^{m_{1}} \lambda^{k-1-r} \varphi_{2}^{*}\left(r-m_{1}\right)\right.
$$$$
\times\left[\lambda^{m_{s}+1} \varphi_{1}^{*}(0)+\left(m_{s}+1\right) \lambda^{m_{s}} \varphi_{2}^{*}(0)\right.
$$$$
+\sum_{r=0}^{m_{s}} \lambda^{m_{s}-r}\left[\sum_{l=s}^{n} b_{12}^{* l} \varphi_{2}^{*}\left(r-m_{l}\right)\right]
$$$$
+\sum_{l=1}^{s-1} b_{12}^{* l}\left[\sum_{r=0}^{m_{l}} \lambda^{m_{s}-r} \varphi_{2}^{*}\left(r-m_{l}\right)\right.
$$$$
\left.\left.+\left(m_{s}-m_{l}\right) \lambda^{m_{s}-m_{l}} \varphi_{2}^{*}(0)\right]\right]
$$

$$
\begin{aligned}
+\sum_{r=m_{s}+1}^{k-1} \lambda^{k-1-r} & {\left[\lambda^{r} \varphi_{2}^{*}(0)+\sum_{l=1}^{s} b_{12}^{* l} \lambda^{r-m_{l}} \varphi_{2}^{*}(0)\right.} \\
& \left.+\sum_{l=s+1}^{n} b_{12}^{* l} \varphi_{2}^{*}\left(r-m_{l}\right)\right]
\end{aligned}
$$

$$
\begin{gathered}
=\lambda^{k} \varphi_{1}^{*}(0)+\left(m_{s}+1\right) \lambda^{k-1} \varphi_{2}^{*}(0) \\
+\sum_{r=0}^{m_{s}} \lambda^{k-1-r}\left[\sum_{l=s}^{n} b_{12}^{* l} \varphi_{2}^{*}\left(r-m_{l}\right)\right] \\
+\sum_{l=1}^{s-1} b_{12}^{* l}\left[\sum_{r=0}^{m_{l}} \lambda^{k-1-r} \varphi_{2}^{*}\left(r-m_{l}\right)\right. \\
\left.+\left(m_{s}-m_{l}\right) \lambda^{k-1-m_{l}} \varphi_{2}^{*}(0)\right] \\
+\left(k-1-m_{s}\right) \lambda^{k-1} \varphi_{2}^{*}(0) \\
+\sum_{r=m_{s}+1}^{k-1} \lambda^{k-1-r}\left[\sum_{l=1}^{s} b_{12}^{* l} \lambda^{r-m_{l}} \varphi_{2}^{*}(0)\right. \\
\left.+\sum_{l=s+1}^{n} b_{12}^{* l} \varphi_{2}^{*}\left(r-m_{l}\right)\right]
\end{gathered}
$$

$$
\left.+\left(m_{s}-m_{1}\right) \lambda^{k-1-m_{1}} \varphi_{2}^{*}(0)\right]
$$$$
+b_{12}^{* 2}\left[\sum_{r=0}^{m_{2}} \lambda^{k-1-r} \varphi_{2}^{*}\left(r-m_{2}\right)\right.
$$$$
\left.+\left(m_{s}-m_{2}\right) \lambda^{k-1-m_{2}} \varphi_{2}^{*}(0)\right]
$$$$
+\cdots
$$$$
+b_{12}^{* s-1}\left[\sum_{r=0}^{m_{s-1}} \lambda^{k-1-r} \varphi_{2}^{*}\left(r-m_{s-1}\right)\right.
$$$$
\left.+\left(m_{s}-m_{s-1}\right) \lambda^{k-1-m_{s-1}} \varphi_{2}^{*}(0)\right]
$$$$
+\left(k-1-m_{s}\right)\left[\lambda^{k-1-m_{1}} b_{12}^{* 1} \varphi_{2}^{*}(0)\right.
$$

$$
+\lambda^{k-1-m_{2}} b_{12}^{* 2} \varphi_{2}^{*}(0)
$$$$
+\cdots+\lambda^{k-1-m_{s-1}} b_{12}^{* s-1} \varphi_{2}^{*}(0)
$$$$
\left.+\lambda^{k-1-m_{2}} b_{12}^{* s} \varphi_{2}^{*}(0)\right]
$$

$$
\begin{aligned}
& =\lambda^{k} \varphi_{1}^{*}(0)+k \lambda^{k-1} \varphi_{2}^{*}(0) \\
& +\sum_{r=0}^{k-1} \lambda^{k-1-r}\left[\sum_{l=s+1}^{n} b_{12}^{* l} \varphi_{2}^{*}\left(r-m_{l}\right)\right] \\
& +b_{12}^{* 1}\left[\sum_{r=0}^{m_{1}} \lambda^{k-1-r} \varphi_{2}^{*}\left(r-m_{1}\right)\right. \\
& \left.+\left(k-1-m_{1}\right) \lambda^{k-1-m_{1}} \varphi_{2}^{*}(0)\right] \\
& +b_{12}^{* 2}\left[\sum_{r=0}^{m_{2}} \lambda^{k-1-r} \varphi_{2}^{*}\left(r-m_{2}\right)\right. \\
& \left.+\left(k-1-m_{2}\right) \lambda^{k-1-m_{2}} \varphi_{2}^{*}(0)\right] \\
& +\cdots \quad b_{12}^{* s-1}\left[\sum_{r=0}^{m_{s-1}} \lambda^{k-1-r} \varphi_{2}^{*}\left(r-m_{s-1}\right)\right. \\
& \left.+\left(k-1-m_{s-1}\right) \lambda^{k-1-m_{s-1}} \varphi_{2}^{*}(0)\right]
\end{aligned}
$$$$
+\sum_{r=0}^{k-1} \lambda^{k-1-r} \times\left[\sum_{l=s+1}^{n} b_{12}^{* l} \varphi_{2}^{*}\left(r-m_{l}\right)\right]
$$ 


$$
\begin{gathered}
+b_{12}^{* s}\left[\sum_{r=0}^{m_{s}} \lambda^{k-1-r} \varphi_{2}^{*}\left(r-m_{s}\right)\right. \\
\left.+\left(k-1-m_{s}\right) \lambda^{k-1-m_{s}} \varphi_{2}^{*}(0)\right] \\
=\lambda^{k} \varphi_{1}^{*}(0)+k \lambda^{k-1} \varphi_{2}^{*}(0) \\
+\sum_{r=0}^{k-1} \lambda^{k-1-r}\left[\sum_{l=s+1}^{n} b_{12}^{* l} \varphi_{2}^{*}\left(r-m_{l}\right)\right] \\
+\sum_{l=1}^{s} b_{12}^{* l}\left[\sum_{r=0}^{m_{l}} \lambda^{k-1-r} \varphi_{2}^{*}\left(r-m_{l}\right)\right. \\
\left.+\left(k-1-m_{l}\right) \lambda^{k-1-m_{l}} \varphi_{2}^{*}(0)\right]
\end{gathered}
$$

In the end, we solve (113) for $k \in \mathbb{Z}_{m_{n}+1}^{\infty}$ with initial data deduced from (122); that is, we consider the problem

$$
\begin{aligned}
y_{1}(k+1)= & \lambda y_{1}(k)+\lambda^{k} \varphi_{2}^{*}(0) \\
& +\sum_{l=1}^{n} b_{12}^{* l} \lambda^{k-m_{l}} \varphi_{2}^{*}(0) \quad \text { if } k \in \mathbb{Z}_{m_{n}+1}^{\infty}, \\
y_{1}\left(m_{n}+1\right)= & \lambda^{m_{n}+1} \varphi_{1}^{*}(0)+\left(m_{n}+1\right) \lambda^{m_{n}} \varphi_{2}^{*}(0) \\
& +\sum_{r=0}^{m_{n}} \lambda^{m_{n}-r} b_{12}^{* n} \varphi_{2}^{*}\left(r-m_{n}\right) \\
& +\sum_{l=1}^{n-1} b_{12}^{* l}\left[\sum_{r=0}^{m_{l}} \lambda^{m_{n}-r} \varphi_{2}^{*}\left(r-m_{l}\right)\right. \\
& \left.+\left(m_{n}-m_{l}\right) \lambda^{m_{n}-m_{l}} \varphi_{2}^{*}(0)\right] .
\end{aligned}
$$

Applying formula (33) yields (for $k \in \mathbb{Z}_{m_{n}+2}^{\infty}$ )

$$
\begin{aligned}
& y_{1}(k) \\
& =\lambda^{k-\left(m_{n}+1\right)} y_{1}\left(m_{n}+1\right) \\
& \quad+\sum_{r=m_{n}+1}^{k-1} \lambda^{k-1-r}\left[\lambda^{r} \varphi_{2}^{*}(0)+\sum_{l=1}^{n} b_{12}^{* l} \lambda^{r-m_{l}} \varphi_{2}^{*}(0)\right]
\end{aligned}
$$

$$
\begin{aligned}
& =\lambda^{k-m_{n}-1}\left[\lambda^{m_{n}+1} \varphi_{1}^{*}(0)+\left(m_{n}+1\right) \lambda^{m_{n}} \varphi_{2}^{*}(0)\right. \\
& +\sum_{r=0}^{m_{n}} \lambda^{m_{n}-r} b_{12}^{* n} \varphi_{2}^{*}\left(r-m_{n}\right) \\
& +\sum_{l=1}^{n-1} b_{12}^{* l}\left[\sum_{r=0}^{m_{l}} \lambda^{m_{n}-r} \varphi_{2}^{*}\left(r-m_{l}\right)\right. \\
& \left.\left.+\left(m_{n}-m_{l}\right) \lambda^{m_{n}-m_{l}} \varphi_{2}^{*}(0)\right]\right] \\
& +\sum_{r=m_{n}+1}^{k-1} \lambda^{k-1-r}\left[\lambda^{r} \varphi_{2}^{*}(0)+\sum_{l=1}^{n} b_{12}^{* l} \lambda^{r-m_{l}} \varphi_{2}^{*}(0)\right] \\
& =\lambda^{k} \varphi_{1}^{*}(0)+\left(m_{n}+1\right) \lambda^{k-1} \varphi_{2}^{*}(0) \\
& +\sum_{r=0}^{m_{n}} \lambda^{k-1-r} b_{12}^{* n} \varphi_{2}^{*}\left(r-m_{n}\right) \\
& +\sum_{l=1}^{n-1} b_{12}^{* l}\left[\sum_{r=0}^{m_{l}} \lambda^{k-1-r} \varphi_{2}^{*}\left(r-m_{l}\right)\right. \\
& \left.+\left(m_{n}-m_{l}\right) \lambda^{k-1-m_{l}} \varphi_{2}^{*}(0)\right] \\
& +\left(k-1-m_{n}\right) \lambda^{k-1} \varphi_{2}^{*}(0) \\
& +\sum_{r=m_{n}+1}^{k-1} \lambda^{k-1-r}\left[\sum_{l=1}^{n} b_{12}^{* l} \lambda^{r-m_{l}} \varphi_{2}^{*}(0)\right] \\
& =\lambda^{k} \varphi_{1}^{*}(0)+k \lambda^{k-1} \varphi_{2}^{*}(0)+\sum_{r=0}^{m_{n}} \lambda^{k-1-r} b_{12}^{* n} \varphi_{2}^{*}\left(r-m_{n}\right) \\
& +b_{12}^{* 1}\left[\sum_{r=0}^{m_{1}} \lambda^{k-1-r} \varphi_{2}^{*}\left(r-m_{1}\right)\right. \\
& \left.+\left(m_{n}-m_{1}\right) \lambda^{k-1-m_{1}} \varphi_{2}^{*}(0)\right] \\
& +b_{12}^{* 2}\left[\sum_{r=0}^{m_{2}} \lambda^{k-1-r} \varphi_{2}^{*}\left(r-m_{2}\right)\right. \\
& \left.+\left(m_{n}-m_{2}\right) \lambda^{k-1-m_{2}} \varphi_{2}^{*}(0)\right]
\end{aligned}
$$




$$
\begin{aligned}
& +b_{12}^{* n-1}\left[\sum_{r=0}^{m_{n-1}} \lambda^{k-1-r} \varphi_{2}^{*}\left(r-m_{n-1}\right)\right. \\
& \left.+\left(m_{n}-m_{n-1}\right) \lambda^{k-1-m_{n-1}} \varphi_{2}^{*}(0)\right] \\
& +\left(k-1-m_{n}\right) \\
& +\left[\lambda^{k-1-m_{1}} b_{12}^{* 1} \varphi_{2}^{*}(0)+\lambda^{k-1-m_{2}} b_{12}^{* 2} \varphi_{2}^{*}(0)\right. \\
& +\cdots+\lambda^{k-1-m_{n-1}} b_{12}^{* n-1} \varphi_{2}^{*}(0) \\
& +\sum_{l=1}^{n} b_{12}^{* l}\left[\sum_{r=0}^{m_{l}} \lambda^{k-1-r} \varphi_{2}^{*}\left(r-m_{l}\right)\right. \\
& \left.+\lambda^{k} \varphi_{1}^{*}(0)+k \lambda^{k-1} \varphi_{2}^{*}(0)\right] \\
& +b_{12}^{* n}\left[\sum_{r=0}^{m_{n}} \lambda^{k-1-r} \varphi_{2}^{*}\left(r-m_{n}\right)\right. \\
& +b_{12}^{* 1}\left[\sum_{r=0}^{m_{1}} \lambda^{k-1-r} \varphi_{2}^{*}\left(r-m_{1}\right)\right. \\
& +{ }_{12}^{* n-1}\left(k \lambda^{k-1} \varphi_{2}^{*}(0)\right. \\
& +\left(k-1-m_{n} \lambda^{k-1-r} \varphi_{2}^{*}\left(r-m_{n-1}\right)\right. \\
& +b_{12}^{* 2}\left[\sum_{r=0}^{m_{2}} \lambda^{k-1-r} \varphi_{2}^{*}\left(r-m_{2}\right)\right. \\
& \left.+\left(k-1-m_{2}\right) \lambda^{k-1-m_{2}} \varphi_{2}^{*-1-m_{1}} \varphi_{2}^{*}(0)\right]
\end{aligned}
$$

Summing up all particular cases (115)-(124), we get

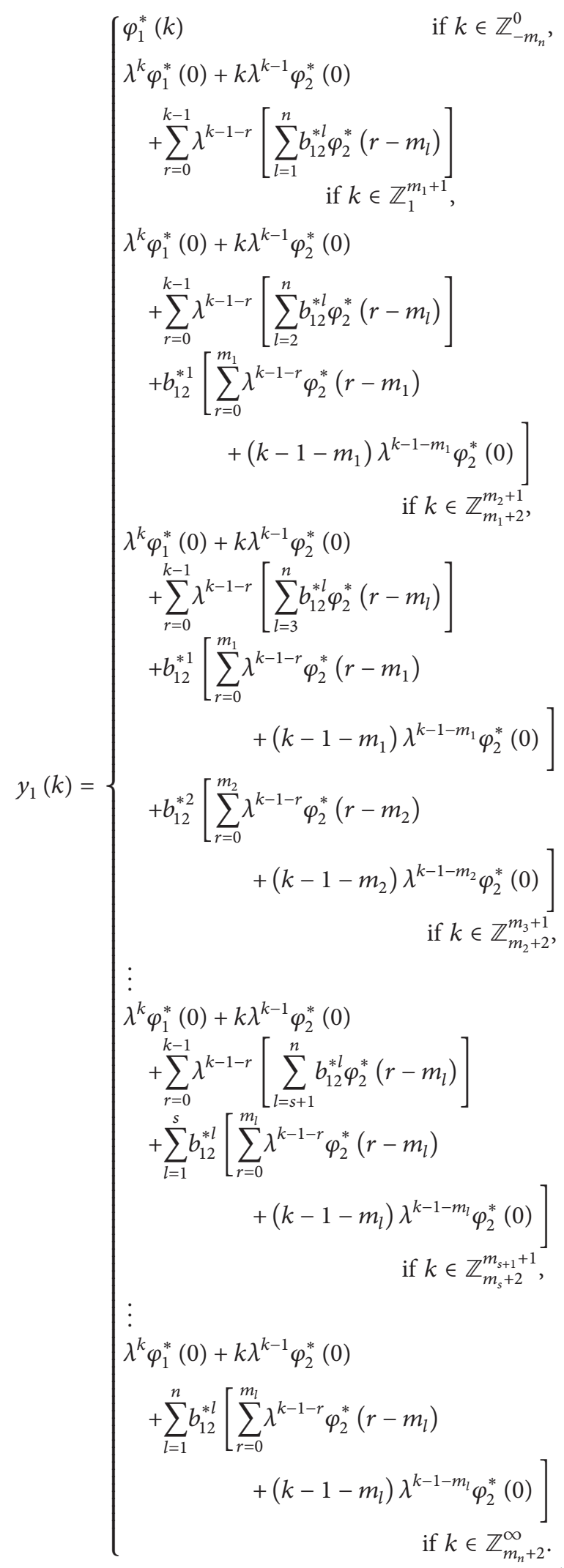

Formulas (108) and (109) are consequences of (125), (112). 


\section{Dimension of the Set of Solutions}

Since all the possible cases of the planar system (1) with weak delay have been analysed, we are ready to formulate results concerning the dimension of the space of solutions of (1) assuming that initial condition (3) is variable. Although case $b_{11}^{* l}=b_{22}^{* l}=b_{12}^{* l}=b_{21}^{* l}=0$ does not lead to a weakly delayed system and is excluded by (2), for completeness of analysis we incorporate such possibility in our analysis as well (such a case can be considered as a degenerated weakly delayed system). Before formulation we remark that if an assumption in the following theorem is assumed to be valid for a fixed index $l \in\{1,2, \ldots, n\}$, it is easy to see that it must be valid for all indices $l=1,2, \ldots, n$.

Theorem 10. Let (1) be a weakly delayed system and let (35) having both roots different from zero and $l \in\{1,2, \ldots, n\}$ be fixed. Then the space of solutions, being initially $2\left(m_{n}+1\right)-$ dimensional, becomes on $\mathbb{Z}_{m_{n}+2}^{\infty}$ only

(1) $\left(m_{n}+2\right)$-dimensional if (35) has

(a) two real distinct roots and $\left(b_{12}^{* l}\right)^{2}+\left(b_{21}^{* l}\right)^{2}>0$,

(b) a double real root, $b_{12}^{* l} b_{21}^{* l}=0$, and $\left(b_{12}^{* l}\right)^{2}+\left(b_{21}^{* l}\right)^{2}>0$.

(c) a double real root and $b_{12}^{* l} b_{21}^{* l} \neq 0$,

(2) 2-dimensional if (35) has

(a) two real distinct roots and $b_{12}^{* l}=b_{21}^{* l}=0$,

(b) a pair of complex conjugate roots,

(c) a double real root and $b_{12}^{* l}=b_{21}^{* l}=0$.

Proof. We will carefully go through all the theorems considered (Theorems 5-9) adding the case of a pair of complex conjugate roots and our conclusion will hold at least on $\mathbb{Z}_{m_{n}+2}^{\infty}$ (some of the statements hold on a larger interval).

(a) Analysing the statement of Theorem 5 (case (36) of two real distinct roots), we obtain the following subcases.

(a1) If $b_{11}^{* l}=b_{22}^{* l}=b_{21}^{* l}=0, b_{12}^{* l} \neq 0$, then the dimension of the space of solutions on $\mathbb{Z}_{m_{n}+2}^{\infty}$ equals $m_{n}+2$ since the last formula in (47) uses only $m_{n}+2$ arbitrary parameters:

$\varphi_{1}^{*}(0), \varphi_{2}^{*}\left(-m_{n}\right), \varphi_{2}^{*}\left(-m_{n}+1\right), \ldots, \varphi_{2}^{*}(0)$.

(a2) If $b_{11}^{* l}=b_{22}^{* l}=b_{12}^{* l}=0, b_{21}^{* l} \neq 0$, then the dimension of the space of solutions on $\mathbb{Z}_{m_{n}+2}^{\infty}$ equals $m_{n}+2$ since the last formula in (48) uses only $m_{n}+2$ arbitrary parameters:

$\varphi_{1}^{*}\left(-m_{n}\right), \varphi_{1}^{*}\left(-m_{n}+1\right), \ldots, \varphi_{1}^{*}(0), \varphi_{2}^{*}(0)$.

(a3) If $b_{12}^{* l}=b_{21}^{* l}=0$, then $b_{11}^{* l}=b_{22}^{* l}=0$ and Theorem 5 is not applicable. The dimension of the space of solutions on $\mathbb{Z}_{m_{n}+2}^{\infty}$ equals 2 since the solution is determined only by 2 arbitrary parameters

$$
\varphi_{1}^{*}(0), \varphi_{2}^{*}(0) \text {. }
$$

This means that all the cases considered are covered by conclusions (1)(a) and (2)(a) of Theorem 10.

(b) In case (37) of two complex conjugate roots, we have $b_{11}^{* l}=b_{22}^{* l}=b_{12}^{* l}=b_{21}^{* l}=0$ (i.e., we deal not with a weakly delayed system, as noted previosly) and the formula (70) uses only 2 arbitrary parameters

$$
\varphi_{1}^{*}(0), \varphi_{2}^{*}(0)
$$

for every $k \in \mathbb{Z}_{1}^{\infty}$. This is covered by case (2)(b) of Theorem 10.

(c) Analysing the statement of Theorems 7 and 8 (case (38) of a double real root), we obtain the following subcases.

(c1) If $b_{21}^{* l}=0, b_{12}^{* l} \neq 0$, then the dimension of the space of solutions on $\mathbb{Z}_{m_{n}+2}^{\infty}$ equals $m_{n}+2$ since the last formula in (76) uses only $m_{n}+2$ arbitrary parameters:

$\varphi_{1}^{*}(0), \varphi_{2}^{*}\left(-m_{n}\right), \varphi_{2}^{*}\left(-m_{n}+1\right), \ldots, \varphi_{2}^{*}(0)$.

(c2) If $b_{12}^{* l}=0, b_{21}^{* l} \neq 0$, then the dimension of the space of solutions on $\mathbb{Z}_{m_{n}+2}^{\infty}$ equals $m_{n}+2$ since the last formula in (77) uses only $m_{n}+2$ arbitrary parameters:

$$
\varphi_{1}^{*}\left(-m_{n}\right), \varphi_{1}^{*}\left(-m_{n}+1\right), \ldots, \varphi_{1}^{*}(0), \varphi_{2}^{*}(0) \text {. }
$$

(c3) If $b_{12}^{* l}=b_{21}^{* l}=0$ (degenerated weakly delayed system), then the dimension of the space of solutions on $\mathbb{Z}_{m_{n}+2}^{\infty}$ equals 2 and solutions are determined only by 2 arbitrary parameters:

$$
\varphi_{1}^{*}(0), \varphi_{2}^{*}(0) .
$$

(c4) If $b_{12}^{* l} b_{21}^{* l} \neq 0$, then the dimension of the space of solutions on $\mathbb{Z}_{m_{n}+2}^{\infty}$ equals $m_{n}+2$ since the last formula in (81) uses only $m_{n}+2$ arbitrary parameters:

$C\left(-m_{n}\right), C\left(-m_{n}+1\right), \ldots, C(0), \varphi_{1}^{*}(0)$,

where

$C(k):=\left[\varphi_{1}^{*}(k)+\frac{b_{12}^{* 1}}{b_{11}^{* 1}} \varphi_{2}^{*}(k)\right], \quad k \in \mathbb{Z}_{-m_{n}}^{0}$.

The parameter $\varphi_{2}^{*}(0)$ cannot be seen as independent since it depends on the independent parameters $\varphi_{1}^{*}(0)$ and $C(0)$.

All the cases considered are covered by conclusions (1)(b), (1)(c), and (2)(c) of Theorem 10.

(d) Analysing the statement of Theorem 9 (case (39) of a double real root), we obtain the following subcases:

(d1) If $b_{11}^{* l}=b_{22}^{* l}=b_{21}^{* l}=0, b_{12}^{* l} \neq 0$, then the dimension of the space of solutions on $\mathbb{Z}_{m_{n}+2}^{\infty}$ equals $m_{n}+2$ since the last formula in (108) uses only $m_{n}+2$ arbitrary parameters:

$\varphi_{1}^{*}(0), \varphi_{2}^{*}\left(-m_{n}\right), \varphi_{2}^{*}\left(-m_{n}+1\right), \ldots, \varphi_{2}^{*}(0)$ 
and the last formula in (109) provides no new information.

(d2) If $b_{11}^{* l}=b_{22}^{* l}=b_{21}^{* l}=b_{12}^{* l}=0$ (degenerated weakly delayed system), then the dimension of the space of solutions on $\mathbb{Z}_{m_{n}+2}^{\infty}$ equals 2 since solutions are determined only by 2 arbitrary parameters

$$
\varphi_{1}^{*}(0), \varphi_{2}^{*}(0) \text {. }
$$

Both cases are covered by conclusions (1)(b) and (2)(c) of Theorem 10.

Since there are no cases other than cases (a)-(d), the proof is finished.

Theorem 10 can be formulated simply as follows.

Theorem 11. Let (1) be a weakly delayed system and let (35) have both roots different from zero, then the space of solutions, being initially $2\left(m_{n}+1\right)$-dimensional, is on $\mathbb{Z}_{m_{n}+2}^{\infty}$ only

(1) $\left(m_{n}+2\right)$-dimensional if $\left(b_{12}^{* l}\right)^{2}+\left(b_{21}^{* l}\right)^{2}>0$,

(2) 2-dimensional if $b_{12}^{* l}=b_{21}^{* l}=0$.

We omit the proofs of the following two theorems since, again, they are much the same as those of Theorems 5-9.

Theorem 12. Let (1) be a weakly delayed system and let (35) have a simple root $\lambda=0$, then the space of solutions, being initially $2\left(m_{n}+1\right)$-dimensional, is either $\left(m_{n}+1\right)$-dimensional or 1-dimensional on $\mathbb{Z}_{m_{n}+2}^{\infty}$.

Theorem 13. Let (1) be a weakly delayed system and let (35) have a double root $\lambda=0$, then the space of solutions, being initially $2\left(m_{n}+1\right)$-dimensional, turns into a 0-dimensional space on $\mathbb{Z}_{m_{n}+2}^{\infty}$, namely, into the zero solution.

\section{Concluding Remarks}

To our best knowledge, weakly delayed systems were firstly defined in [9] for systems of linear delayed differential systems with constant coefficients and in [1] for planar linear discrete systems with a single delay (in these papers such systems are called systems with a weak delay). The weakly delayed systems analyzed in this paper can be simplified and their solutions can be found in explicit analytical forms (results obtained generalize those in $[1,2])$. Consequently, analytical forms of solutions can be used directly to solve several problems for weakly delayed systems, for example, problems of asymptotical behavior of their solutions, boundary-value problems, or some problems of control theory (using different methods, such problems have recently been investigated e.g., in [10-18]). For an alternative approach to differentialdifference equations using the variational iteration method and new analytical and asymptotic methods see, for example, [19-21].

In the case of discrete systems of two equations investigated in this paper, to obtain the corresponding eigenvalues, it is sufficient to solve only a second-order polynomial equation rather than a polynomial equation of order $2 m_{n}$.

\section{Conflict of Interests}

The authors declare that there is no conflict of interests regarding the publication of this paper.

\section{Acknowledgments}

The first author was supported by Grant no. P201/10/1032 of the Czech Grant Agency (Prague). The second author was supported by Grant no. FEKT-S-14-2200 of the Faculty of Electrical Engineering and Communication, Brno University of Technology.

\section{References}

[1] J. Diblík, D. Ya. Khusainov, and Z. Šmarda, "Construction of the general solution of planar linear discrete systems with constant coefficients and weak delay," Advances in Difference Equations, vol. 2009, Article ID 784935, 18 pages, 2009.

[2] J. Diblík and H. Halfarová, "Explicit general solution of planar linear discrete systems with constant coefficients and weak delays," Advances in Difference Equations, vol. 2013, article 50, 29 pages, 2013.

[3] S. Elaydi, An Introduction to Difference Equations, Springer, New York, NY, USA, 3rd edition, 2005.

[4] S. Stević, "More on a rational recurrence relation," Applied Mathematics E-Notes, vol. 4, pp. 80-85, 2004.

[5] S. Stević, "On a system of difference equations," Applied Mathematics and Computation, vol. 218, no. 7, pp. 3372-3378, 2011.

[6] S. Stević, "On some solvable systems of difference equations," Applied Mathematics and Computation, vol. 218, no. 9, pp. 50105018, 2012.

[7] S. Stević, "On the difference equation $x_{n}=x_{n-k} /(b+$ $\left.c x_{n-1} \cdots x_{n-k}\right)$," Applied Mathematics and Computation, vol. 218, no. 11, pp. 6291-6296, 2012.

[8] S. Stević, J. Diblík, B. Iričanin, and Z. Šmarda, "On a thirdorder system of difference equations with variable coefficients," Abstract and Applied Analysis, vol. 2012, Article ID 508523, 22 pages, 2012.

[9] D. Ya. Khusainov, D. B. Benditkis, and J. Diblik, "Weak delay in systems with an aftereffect," Functional Differential Equations, vol. 9, no. 3-4, pp. 385-404, 2002.

[10] J. Čermák, "The stability and asymptotic properties of the $\Theta$-methods for the pantograph equation," IMA Journal of Numerical Analysis, vol. 31, no. 4, pp. 1533-1551, 2011.

[11] J. Čermák and S. Dvořáková, "Boundedness and asymptotic properties of solutions of some linear and sublinear delay difference equations," Applied Mathematics Letters, vol. 25, no. 5, pp. 813-817, 2012.

[12] J. Čermák and M. Urbánek, "On the asymptotics of solutions of delay dynamic equations on time scales," Mathematical and Computer Modelling, vol. 46, no. 3-4, pp. 445-458, 2007.

[13] J. Diblík, "Anti-Lyapunov method for systems of discrete equations," Nonlinear Analysis A, vol. 57, no. 7-8, pp. 1043-1057, 2004.

[14] T. Khokhlova and M. Kipnis, "Numerical and qualitative stability analysis of ring and linear neural networks with a large number of neurons," International Journal of Pure and Applied Mathematics, vol. 76, pp. 403-420, 2012.

[15] T. Khokhlova, M. Kipnis, and V. Malygina, "The stability cone for a delay differential matrix equation," Applied Mathematics Letters, vol. 24, no. 5, pp. 742-745, 2011. 
[16] M. Kipnis and D. Komissarova, "Stability of a delay difference system," Advances in Difference Equations, vol. 2006, Article ID 31409, 9 pages, 2006.

[17] E. Liz and M. Pituk, "Asymptotic estimates and exponential stability for higher-order monotone difference equations," Advances in Difference Equations, no. 1, pp. 41-55, 2005.

[18] Ch. G. Philos and I. K. Purnaras, "An asymptotic result for some delay difference equations with continuous variable," Advances in Difference Equations, no. 1, pp. 1-10, 2004.

[19] N. Faraz, Y. Khan, and F. Austin, "An alternative approach to differential-difference equations using the variational iteration method," Journal of Physical Sciences, vol. 65, no. 12, pp. 10551059, 2010.

[20] Y. Khan, H. Vázquez-Leal, and N. Faraz, "An auxiliary parameter method using Adomian polynomials and Laplace transformation for nonlinear differential equations," Applied Mathematical Modelling, vol. 37, no. 5, pp. 2702-2708, 2013.

[21] Y. Khan and Q. Wu, "Homotopy perturbation transform method for nonlinear equations using He's polynomials," Computers \& Mathematics with Applications, vol. 61, no. 8, pp. 19631967, 2011. 


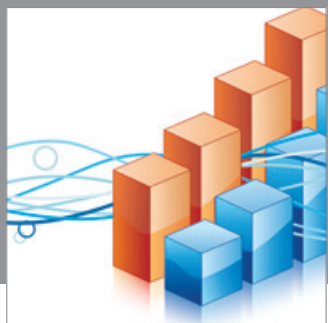

Advances in

Operations Research

mansans

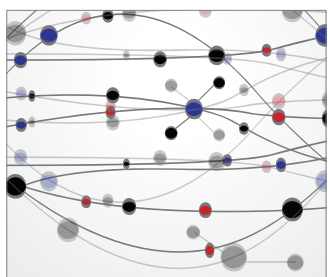

The Scientific World Journal
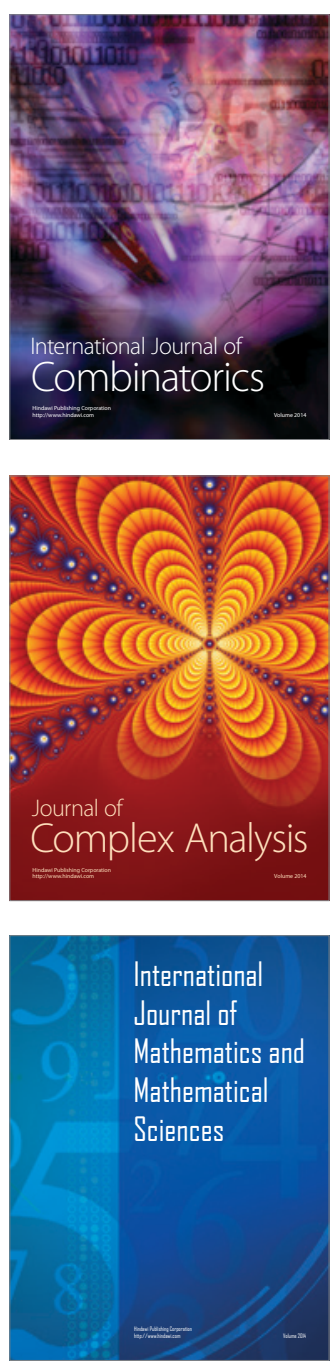
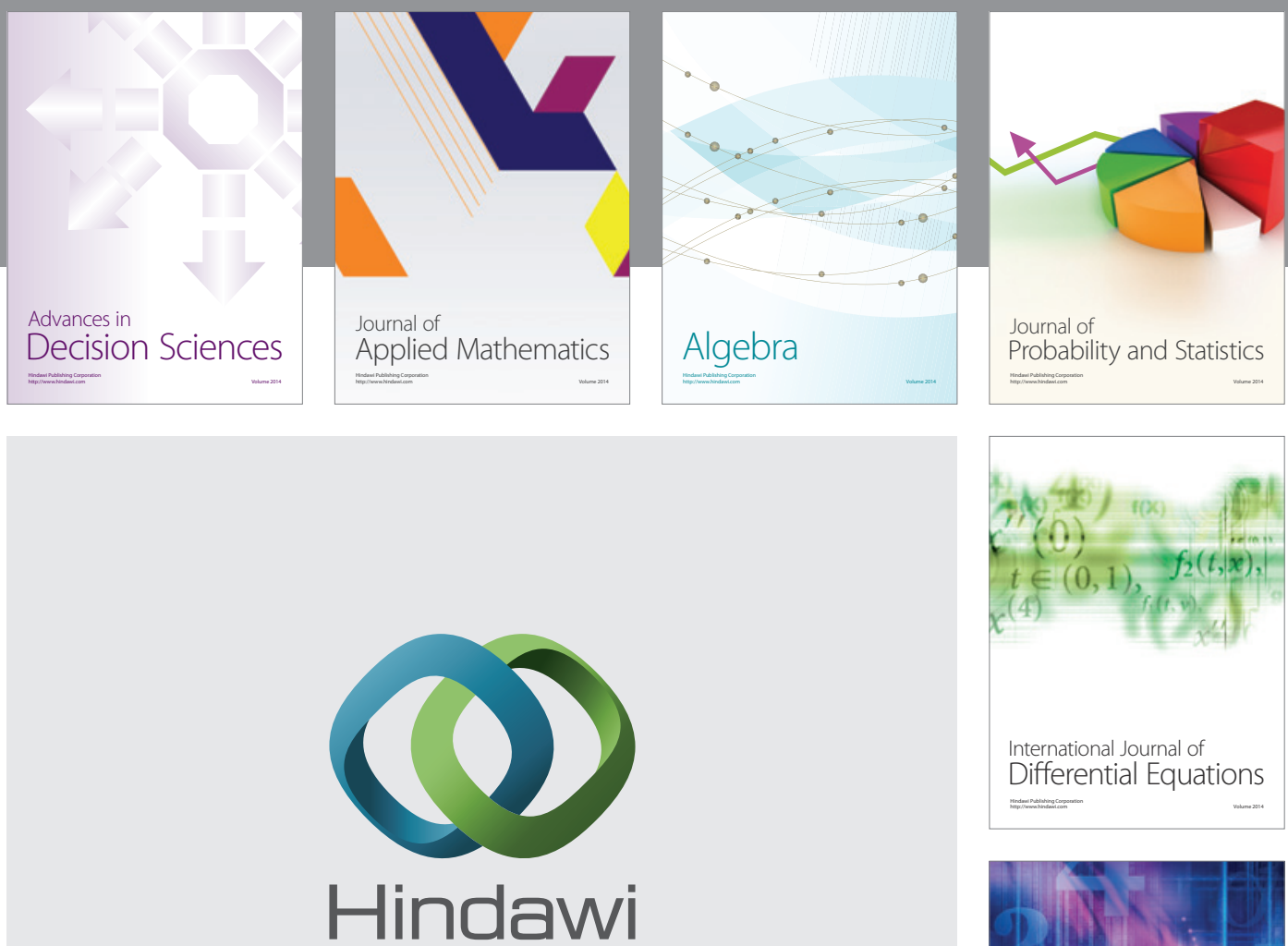

Submit your manuscripts at http://www.hindawi.com
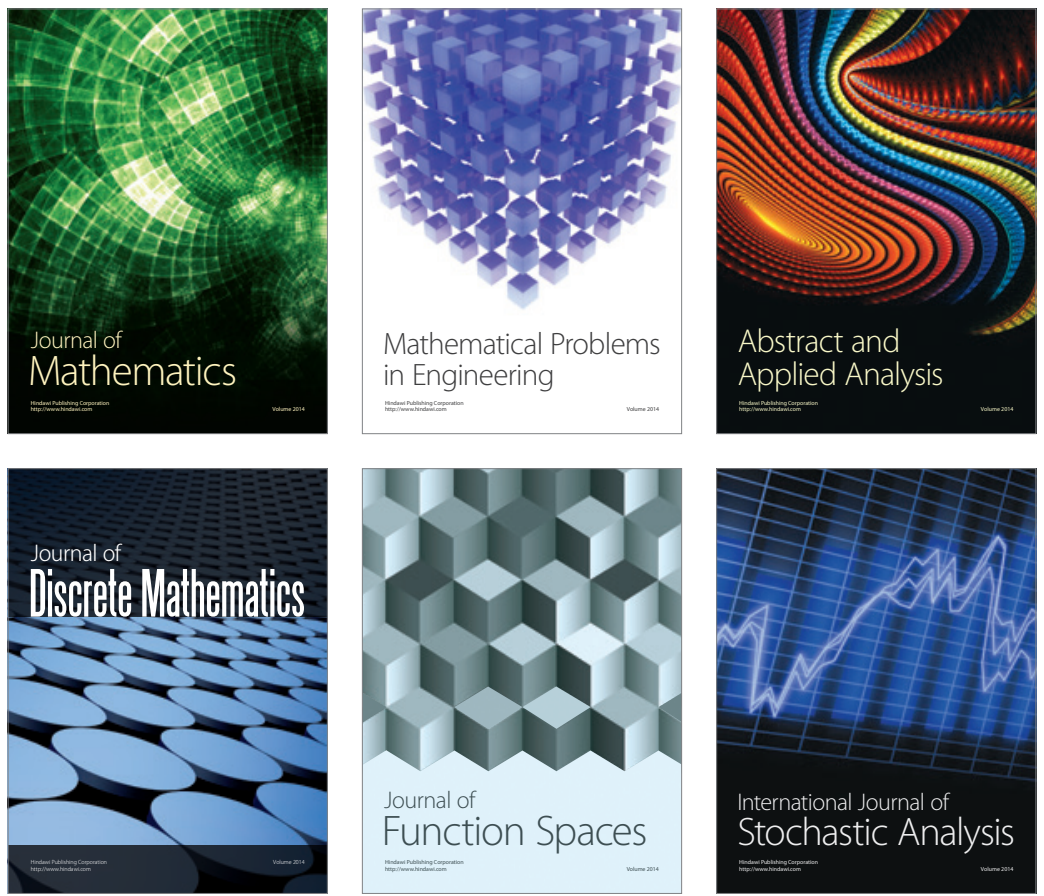

Journal of

Function Spaces

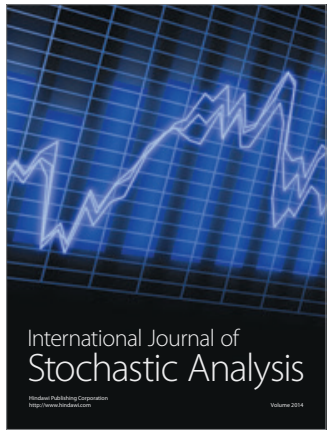

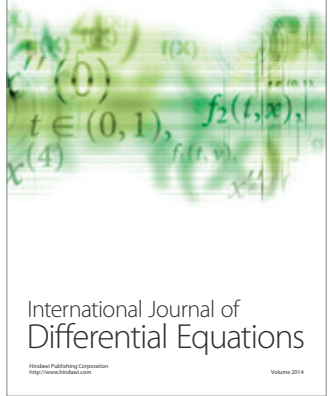
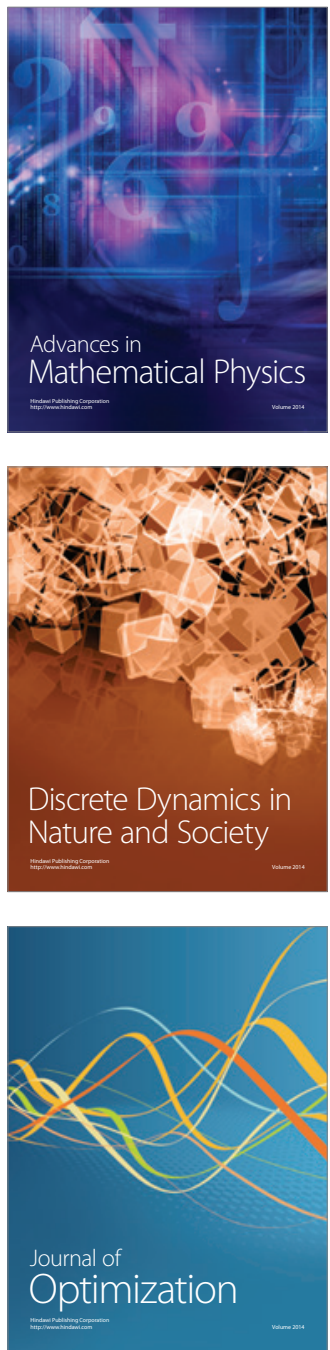